Aus der Abteilung Palliativmedizin

(Prof. Dr. med. F. Nauck)

im Zentrum Anaesthesiologie, Rettungs- und Intensivmedizin

der Medizinischen Fakultät der Universität Göttingen

\title{
Charakteristika von Palliativpatienten mit Atemnot - Ergebnisse der Hospiz- und Palliativerhebungen (HOPE) von 2006 bis 2008
}

\author{
INAUGURAL-DISSERTATION \\ zur Erlangung des Doktorgrades \\ der Medizinischen Fakultät der \\ Georg-August-Universität zu Göttingen
}

vorgelegt von

Nadine Altfelder, geb. Huhnold

aus

Göttingen

Göttingen 2012 
Dekan: Prof. Dr. med. C. Frömmel

I. Berichterstatter: Prof. Dr. med. F. Nauck

II. Berichterstatter/ in:

III. Berichterstatter/ in:

Tag der mündlichen Prüfung: 


\section{Inhaltsverzeichnis}

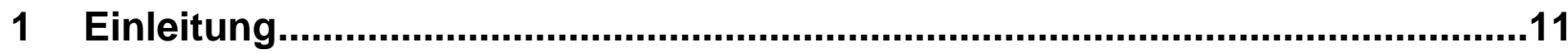

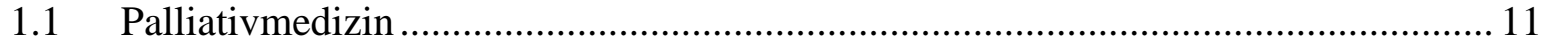

1.1.1 Palliativmedizin nach der Definition der World Health Organization (WHO) ................ 11

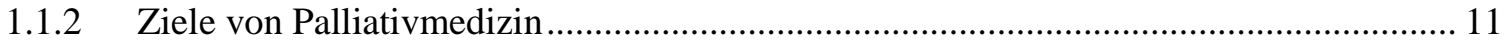

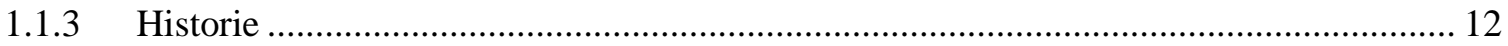

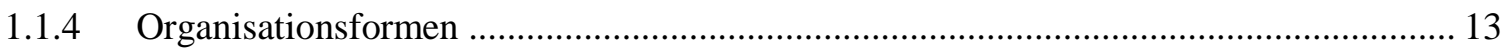

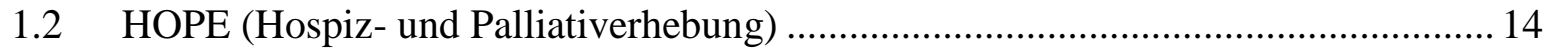

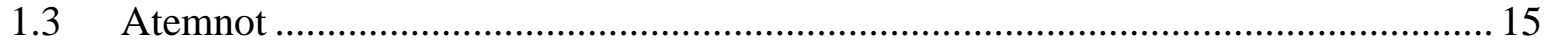

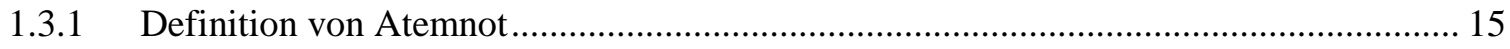

1.3.2 Atemnot bei Palliativpatienten: Prävalenz und ursächliche Erkrankungen ...................... 15

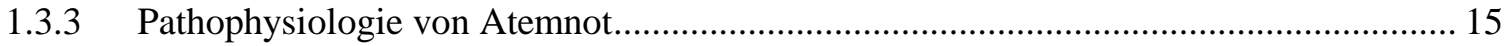

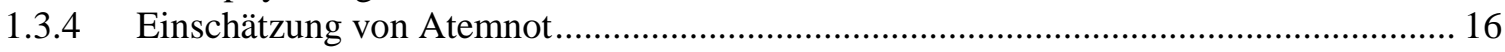

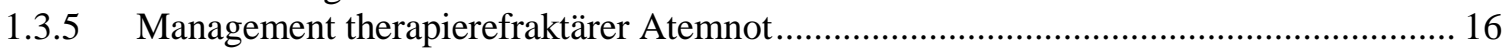

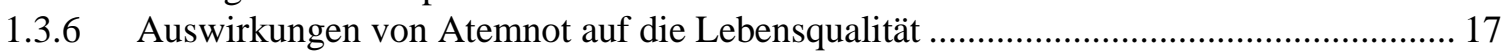

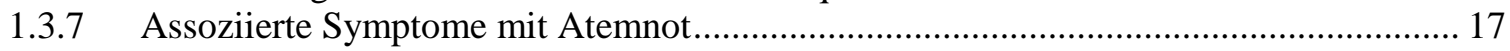

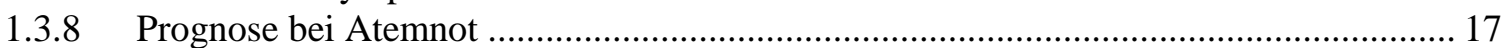

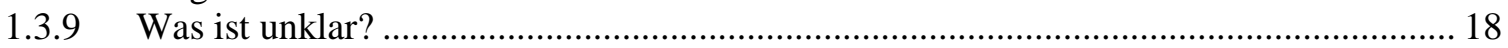

$1.4 \quad$ Fragestellungen der vorliegenden Arbeit .............................................................. 19

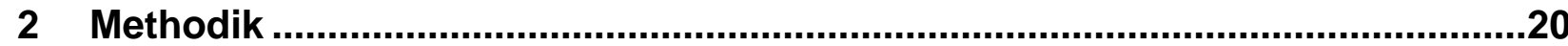

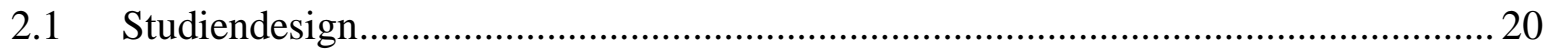

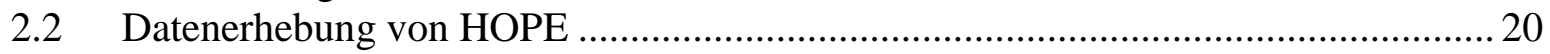

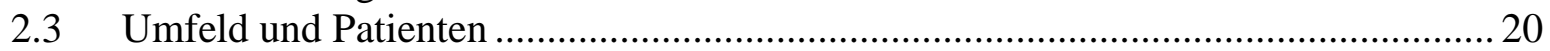

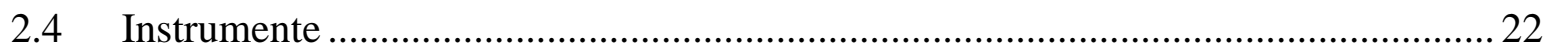

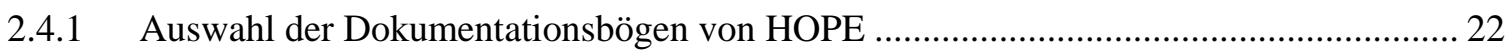

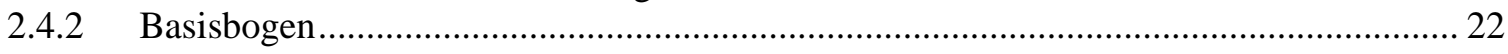

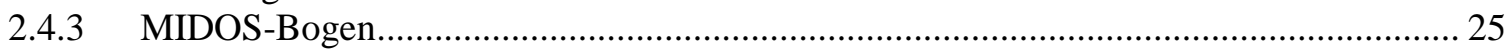

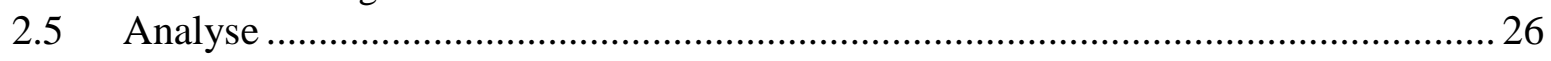

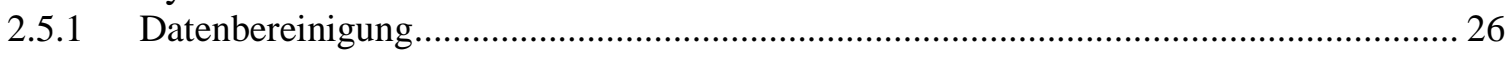

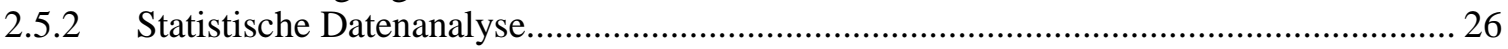

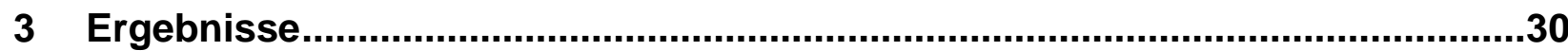

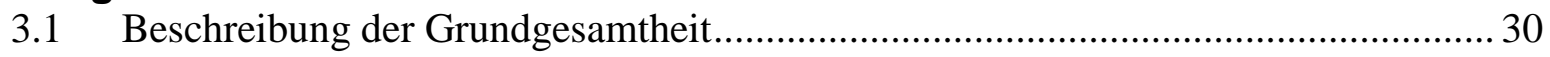

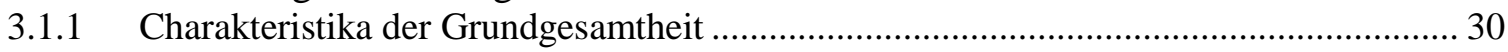

3.1.2 Das Symptom Atemnot in der Grundgesamtheit ....................................................... 32

3.2 Charakteristika von Palliativpatienten mit Atemnot .............................................. 33

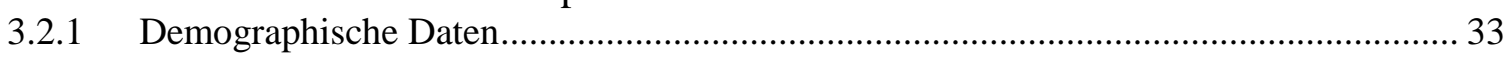

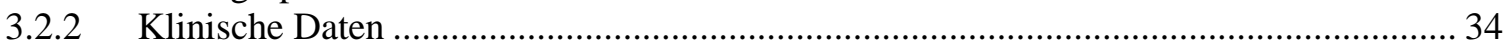

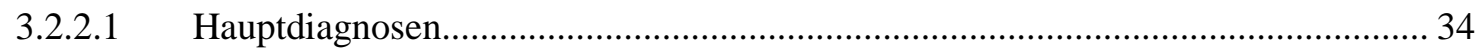

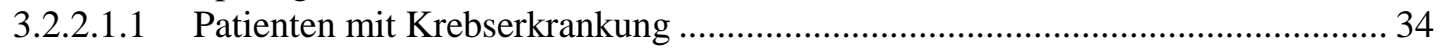

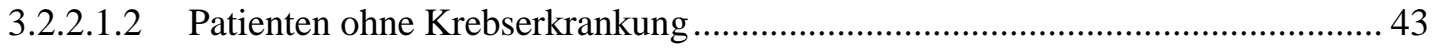

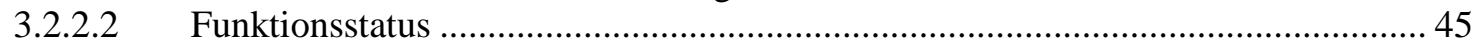

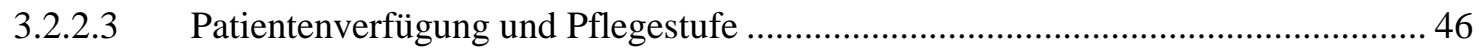

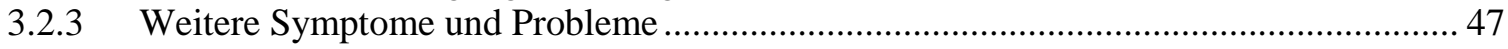

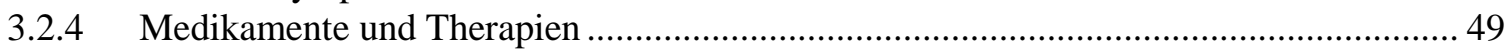

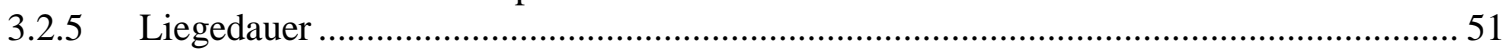

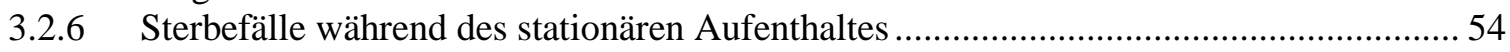

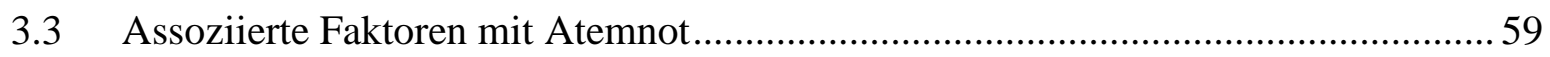

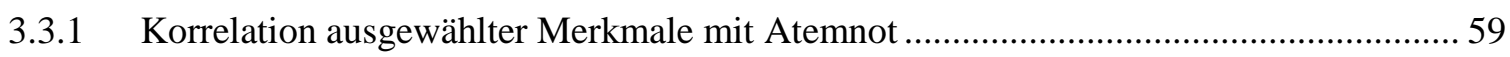




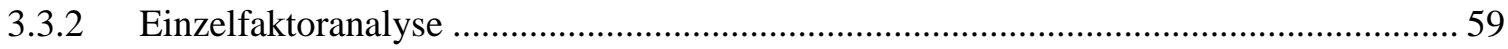

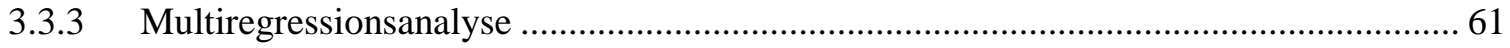

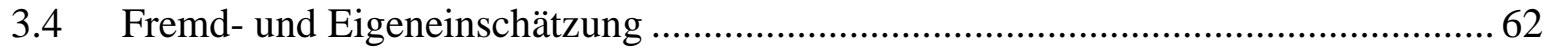

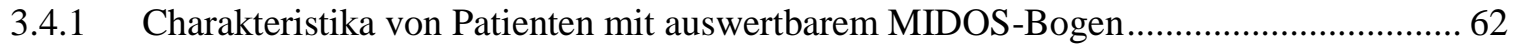

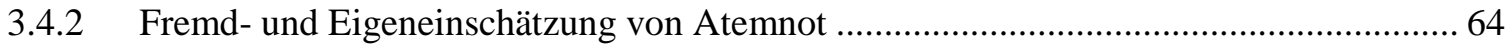

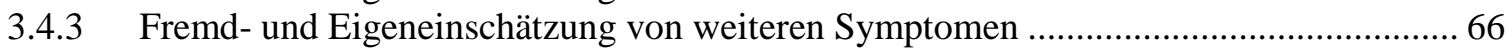

3.4.4 Fremd- und Eigeneinschätzung von Atemnot bei Patienten mit unterschiedlichem

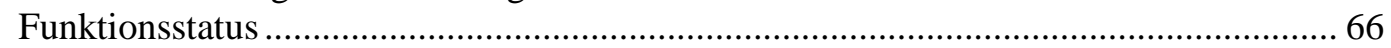

3.4.5 Fremd- und Eigeneinschätzung von Atemnot durch unterschiedliche Professionen....... 67

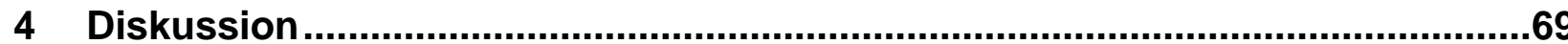

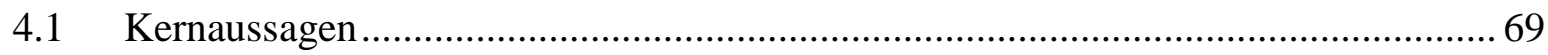

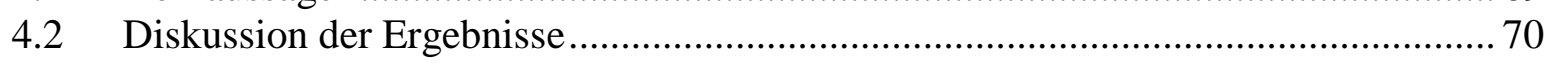

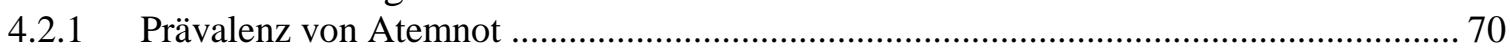

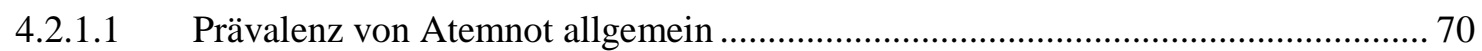

4.2.1.2 Prävalenz von Atemnot in ausgewählten Subgruppen der Grundgesamtheit ........... 70

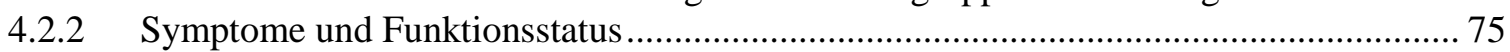

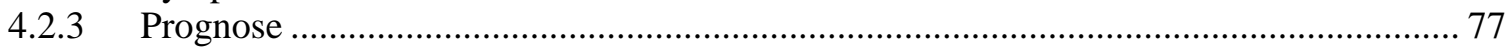

4.2.4 Assoziation von Atemnot mit anderen Symptomen ................................................... 78

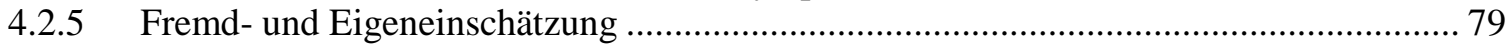

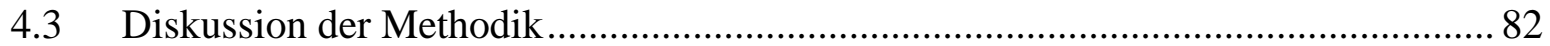

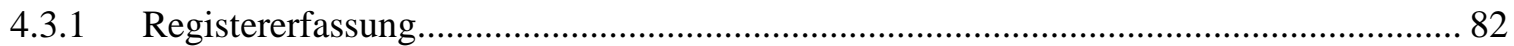

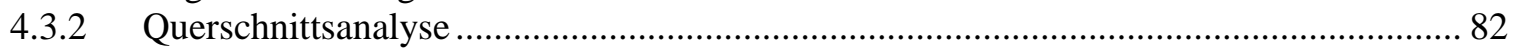

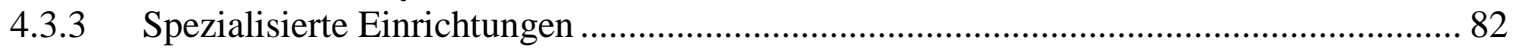

4.3.4 Fremdeinschätzung als Grundlage der Erfassung von Atemnot...................................... 83

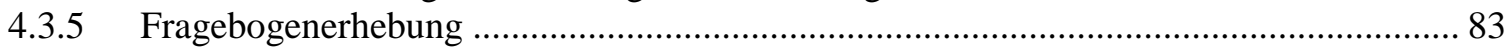

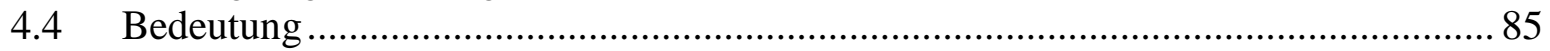

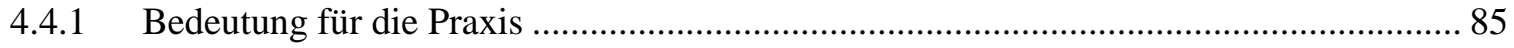

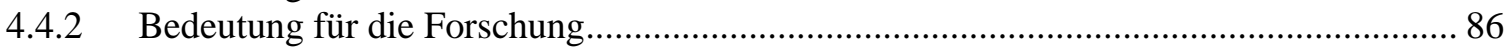

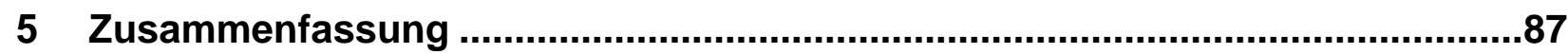

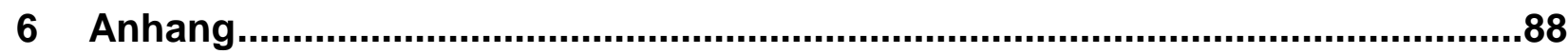

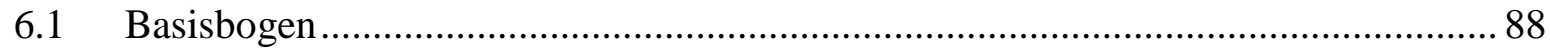

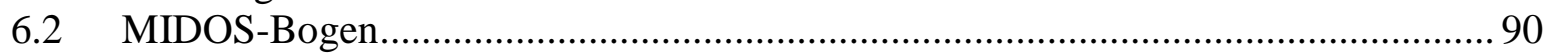

6.3 Bestätigung der Ethikkommission..................................................................... 91

$6.4 \quad$ Weitere Ergebnistabellen .............................................................................. 92

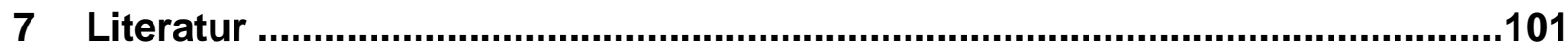




\section{Abbildungsverzeichnis}

Abbildung 1: Auswahl der Patienten von HOPE für die vorliegende Arbeit ..................................... 21

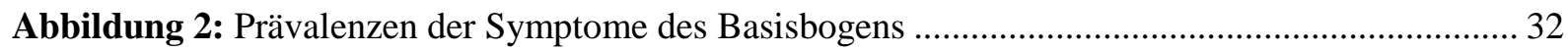

Abbildung 3: Häufigkeit unterschiedlicher Atemnotintensitäten in Abhängigkeit vom Vorhandensein einer Krebserkrankung

Abbildung 4: Vorkommen der 10 häufigsten Krebserkrankungen von Patienten mit Atemnot bei Patienten mit und ohne Atemnot 38

Abbildung 5: Häufigkeit von Metastasen bei Patienten mit und ohne Atemnot................................. 42

Abbildung 6: Funktionsstatus von Patienten mit und ohne Atemnot.................................................. 45

Abbildung 7: Symptomprävalenzen bei Patienten mit und ohne Atemnot ........................................... 47

Abbildung 8: Vergleich der Symptomlast von Patienten mit und ohne Atemnot................................ 48

Abbildung 9: Vergleich der Intensitätslast von Patienten mit und ohne Atemnot.............................. 48

Abbildung 10: Häufigkeit von Problemen bei Patienten mit und ohne Atemnot ............................... 49

Abbildung 11: Häufigkeit der Verordnung von Medikamenten bei Patienten mit und ohne Atemnot bei Aufnahme in eine spezialisierte stationäre Palliativeinrichtung................................................... 50

Abbildung 12: Häufigkeit von Maßnahmen und Therapien bei Patienten mit und ohne Atemnot...... 51

Abbildung 13: Liegedauer verstorbener Patienten mit und ohne Atemnot......................................... 52

Abbildung 14: Liegedauer verstorbener Patienten mit unterschiedlichen Atemnotintensitäten .......... 54

Abbildung 15: Anteil der Todesfälle in den Patientengruppen mit unterschiedlichen

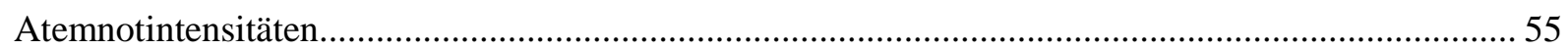

Abbildung 16: Kaplan-Meier-Modell für die Überlebenszeit von Patienten mit und ohne Atemnot .. 56

Abbildung 17: Kaplan-Meier-Modell für die Überlebenszeit von Patienten mit unterschiedlichen Atemnotintensitäten. 57

Abbildung 18: Grad der Übereinstimmung (Cohen's $\kappa$ ) zwischen Selbst- und Fremdeinschätzung für das Vorhandensein und die Intensität von Atemnot in Abhängigkeit vom Funktionsstatus . 67

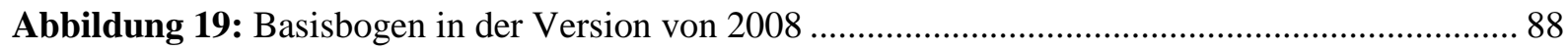

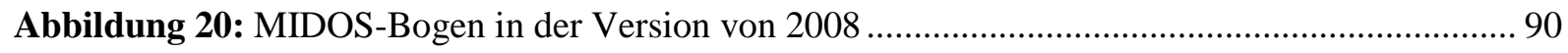

Abbildung 21: Bestätigung der Ethikkommission ............................................................................... 91 


\section{Tabellenverzeichnis}

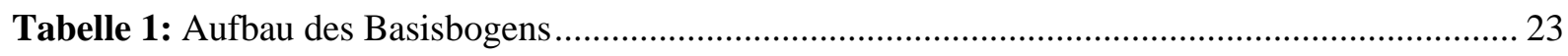

Tabelle 2: Klassifikation der Eastern Cooperative Oncology Group (ECOG)................................... 23

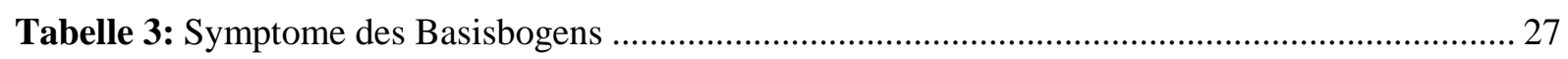

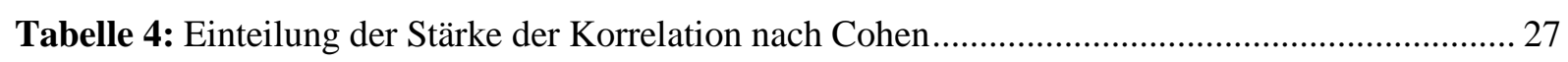

Tabelle 5: Bewertung des Grads der Übereinstimmung nach Cohen's Kappa.................................... 29

Tabelle 6: Anteil der an HOPE teilnehmenden spezialisierten stationären Palliativeinrichtungen ....... 30

Tabelle 7: Demographische Daten der Grundgesamtheit und in den unterschiedlichen Erhebungsjahrgängen ....

Tabelle 8: Verlauf bei den Patienten der Grundgesamtheit und in den unterschiedlichen Einrichtungen

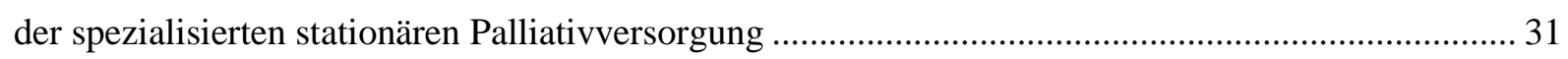

Tabelle 9: Vergleich der demographischen Daten von Patienten mit und ohne Atemnot................... 33

Tabelle 10: Prävalenz von Atemnot in Abhängigkeit von Alter, Geschlecht und Institution .............. 34

Tabelle 11: Häufigkeit von Krebsdiagnosen bei Patienten mit und ohne Atemnot............................... 34

Tabelle 12: Atemnotprävalenz in Abhängigkeit von einer malignen Erkrankung ............................... 35

Tabelle 13: Atemnotintensitäten bei Patienten mit und ohne Krebserkrankung .................................. 35

Tabelle 14: Die häufigsten Krebsdiagnosen bei Patienten mit und ohne Atemnot .............................. 36

Tabelle 15: Die häufigsten Krebsdiagnosen bei Patienten mit Atemnot in Abhängigkeit vom

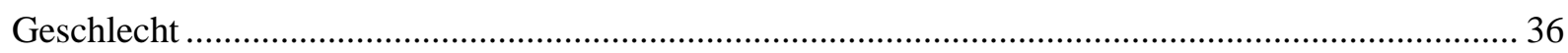

Tabelle 16: Häufigkeit des Bronchialkarzinoms bei Patienten mit und ohne Atemnot, auch in Abhängigkeit vom Geschlecht .............................................................................................. 37

Tabelle 17: Geschlechtsspezifische Atemnotprävalenzen in Abhängigkeit vom Vorliegen eines Bronchialkarzinoms.

Tabelle 18: Atemnotprävalenzen bei den 10 häufigsten Tumorentitäten von Patienten mit Atemnot. 39

Tabelle 19: Anteil der Patienten mit pulmonalen oder pleuralen Metastasen bei den 10 häufigsten Tumorentitäten von Patienten mit Atemnot 39

Tabelle 20: Häufigkeit des kolorektalen Karzinoms bei Krebspatienten mit und ohne Atemnot in Abhängigkeit vom Vorhandensein pulmonaler oder pleuraler Metastasen.

Tabelle 21: Atemnotprävalenzen bei Krebspatienten mit und ohne Lungen- oder Pleurametastasen, auch in Abhängigkeit vom Vorliegen eines kolorektalen Karzinoms

Tabelle 22: Häufigkeit von Metastasen bei Krebspatienten mit und ohne Atemnot

Tabelle 23: Atemnotprävalenzen in Abhängigkeit vom Vorhandensein bzw. der Lokalisation von Metastasen. 42

Tabelle 24: Atemnotprävalenzen in Abhängigkeit vom Vorhandensein einer bösartigen Neubildung in der Lunge. 
Tabelle 25: Die häufigsten Nicht-Krebs-Diagnosen von Patienten mit Atemnot

Tabelle 26: Häufigkeit von COPD und chronischer Herzinsuffizienz bei Patienten ohne

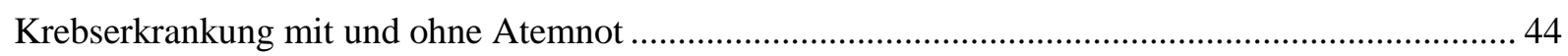

Tabelle 27: Atemnotprävalenzen bei COPD und chronischer Herzinsuffizienz ...............................44

Tabelle 28: Häufigkeit von Lungenerkrankungen bei Patienten mit und ohne Atemnot. .................. 44

Tabelle 29: Funktionsstatus von Patienten mit und ohne Atemnot ...............................................45

Tabelle 30: Atemnotprävalenzen in Abhängigkeit vom Funktionsstatus ........................................ 46

Tabelle 31: Pflegestufen bei Patienten mit und ohne Atemnot ................................................. 46

Tabelle 32: Symptom- und Intensitätslasten von Patienten mit und ohne Atemnot......................... 48

Tabelle 33: Liegedauer von Patienten mit und ohne Atemnot, auch in Abhängigkeit vom Verlauf.... 52

Tabelle 34: Liegedauer von Patienten mit unterschiedlichen Atemnotintensitäten, auch in

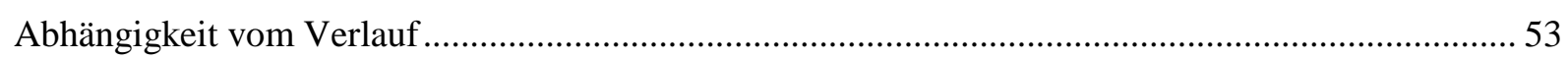

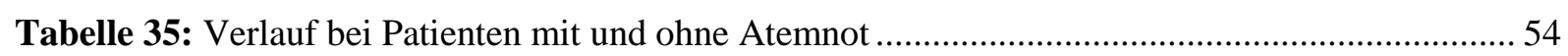

Tabelle 36: Verlauf bei Patienten mit unterschiedlichen Atemnotintensitäten ................................55

Tabelle 37: Vorkommen und Intensität von Atemnot in Abhängigkeit vom Verlauf .........................5 57

Tabelle 38: Risiko zu versterben in Abhängigkeit vom Vorhandensein bzw. der Intensität von

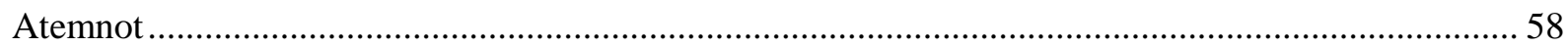

Tabelle 39: Einzelfaktoranalyse ausgewählter demographischer und klinischer Faktoren bzgl. des Risikos, an Atemnot zu leiden. 60

Tabelle 40: Einzelfaktoranalyse ausgewählter Symptome bzgl. des Risikos, an Atemnot zu leiden... 60

Tabelle 41: Multiregressionsanalyse mit ausgewählten Faktoren bzgl. des Risikos, an Atemnot zu leiden 61

Tabelle 42: Tests zur Bewertung der Multiregressionsanalyse 61

Tabelle 43: Charakteristika von Patienten mit und ohne MIDOS-Bogen 63

Tabelle 44: Symptom- und Intensitätslast in Fremd- bzw. Eigeneinschätzung bei Patienten mit und ohne MIDOS-Bogen. 64

Tabelle 45: Atemnotprävalenzen in Fremd- und Eigeneinschätzung 64

Tabelle 46: Vergleich von Fremd- und Eigeneinschätzung für das Vorkommen von Atemnot ..... 65

Tabelle 47: Vergleich von Fremd- und Eigeneinschätzung für die Atemnotintensität ..... 65

Tabelle 48: Grad der Übereinstimmung (Cohen's $\kappa)$ zwischen Selbst- und Fremdeinschätzung für das Vorhandensein und die Intensität der Symptome des MIDOS-Bogens

Tabelle 49: Grad der Übereinstimmung (Cohen's $\kappa)$ zwischen Selbst- und Fremdeinschätzung für das Vorhandensein und die Intensität von Atemnot in Abhängigkeit vom Funktionsstatus 66

Tabelle 50: Grad der Übereinstimmung zwischen Selbst- und Fremdeinschätzung für das Vorhandensein und die Intensität von Atemnot in Abhängigkeit von der Profession. .......................68

Tabelle 51: Prävalenzen der Symptome des Basisbogens in der Grundgesamtheit 92 
Tabelle 52: Vergleich der häufigsten Krebsdiagnosen von Patienten mit Atemnot bei Patienten mit und ohne Atemnot .93

Tabelle 53: Häufigkeiten der Symptome des Basisbogens bei Patienten mit und ohne Atemnot........ 94

Tabelle 54: Vergleich der Probleme von Patienten mit und ohne Atemnot ....................................... 96

Tabelle 55: Vergleich der Medikamente von Patienten mit und ohne Atemnot bei Aufnahme in eine spezialisierte stationäre Palliativeinrichtung ...................................................................................... 97

Tabelle 56: Vergleich der Maßnahmen und Therapien bei Patienten mit und ohne Atemnot ............. 98

Tabelle 57: Korrelationen ausgewählter Merkmale mit dem Vorhandensein bzw. der Intensität von

Atemnot 99 


\section{Abkürzungsverzeichnis}

\begin{tabular}{|c|c|}
\hline ADL & Activities of daily living (Aktivitäten des täglichen Lebens) \\
\hline AIDS & Acquired Immune Deficiency Syndrome (erworbenes Immundefektsyndrom) \\
\hline ALS & Amyotrophe Lateralsklerose \\
\hline bzgl. & bezüglich \\
\hline bzw. & beziehungsweise \\
\hline ca. & circa \\
\hline CDS & Cancer Dyspnoea Scale \\
\hline $\mathrm{CHF}$ & chronical heart failure (chronische Herzinsuffizienz) \\
\hline CI & Konfidenzintervall \\
\hline CLARA & Clinical Analysis, Research and Application \\
\hline COOP & Cooperative \\
\hline COPD & chronisch obstruktive Lungenerkrankung \\
\hline CRQ & Chronic Respiratory Disease Questionaire \\
\hline $\mathrm{d}$ & days (Tage) \\
\hline d. h. & das heißt \\
\hline DGP & Deutsche Gesellschaft für Palliativmedizin \\
\hline DKG & Deutsche Krebsgesellschaft \\
\hline Dr. med. & Doktor der Medizin \\
\hline ECOG & Eastern Cooperative Oncology Group \\
\hline e. d. & epidural \\
\hline et al. & et alii \\
\hline Ex & exkludiert \\
\hline FS & Funktionsstatus \\
\hline geb. & geboren \\
\hline GG & Grundgesamtheit \\
\hline HOPE & Hospiz- und Palliativerhebung \\
\hline I & Intensität \\
\hline ICD & International Classification of Diseases \\
\hline ID & Identifikationsnummer \\
\hline i. th. & intrathekal \\
\hline i. v. & intravenös \\
\hline $\mathrm{K}$ & Kappa \\
\hline k. A. & keine Angabe \\
\hline LGS & Leber-Gallenwegssystem \\
\hline LK & Lymphknoten \\
\hline männl. & männlich \\
\hline $\max$. & maximal \\
\hline MDRS & Motor Neurone Disease Dyspnoea Rating Scale \\
\hline Met. & Metastasen \\
\hline MIDOS & Minimales Dokumentationssystem \\
\hline MRR & Mund- oder Rachenraum \\
\hline MRT & Magnet-Resonanz-Tomographie \\
\hline multimod. & multimodal \\
\hline $\mathrm{N}$ & Anzahl \\
\hline NA & Nadine Altfelder \\
\hline n. a. & nicht auswertbar \\
\hline NRS & Numerical Rating Scale \\
\hline n. s. & nicht signifikant \\
\hline $\begin{array}{l}\text { onkol. } \\
\text { OR }\end{array}$ & $\begin{array}{l}\text { onkologisch } \\
\text { Odds Ratio }\end{array}$ \\
\hline
\end{tabular}




$\begin{array}{ll}\text { p } & \text { Signifikanzniveau } \\ \text { PaP-S } & \text { Palliative Prognostic Score } \\ \text { PET } & \text { Positronen-Emissions-Tomographie } \\ \text { PPI } & \text { Palliative Prognostic Index } \\ \text { Prof. } & \text { Professor } \\ \text { PS } & \text { Palliativstation } \\ \text { RCT } & \text { randomisierte kontrollierte Studie } \\ \text { s. c. } & \text { subkutan } \\ \text { SD } & \text { Standardabweichung } \\ \text { SH } & \text { Stationäres Hospiz } \\ \text { SPSS } & \text { Statistik- Programm- System für Sozialwissenschaften } \\ \text { St. } & \text { Saint (Sankt) } \\ \text { Stat. Hospiz } & \text { stationäres Hospiz } \\ \text { StS } & \text { Steffen Simon } \\ \text { u. a. } & \text { und andere } \\ \text { UK } & \text { United Kingdom } \\ \text { v. a. } & \text { vor allem } \\ \text { VAS } & \text { Visual Analog Scale } \\ \text { vs. } & \text { versus } \\ \text { weibl. } & \text { weiblich } \\ \text { WHO } & \text { World Health Organization } \\ \text { WONCA } & \text { World Organization of National Colleges, Academies } \\ \text { z. B. } & \text { zum Beispiel } \\ \text { ZVK } & \text { zentraler Venenkatheter }\end{array}$




\section{Einleitung}

\subsection{Palliativmedizin}

\subsubsection{Palliativmedizin nach der Definition der World Health Organization (WHO)}

Nach Definition der WHO dient die Palliativmedizin dem Erhalt und der Verbesserung der Lebensqualität von Patienten, die an einer unheilbaren und progredienten Erkrankung mit begrenzter Lebenserwartung leiden. Diese Ziele sollen durch Prävention und Linderung von Beschwerden erreicht werden, indem Schmerzen und andere belastende Symptome früh erkannt und bestmöglich behandelt werden. Palliativmedizin bezieht sich neben körperlichen Beschwerden auch auf Probleme psychosozialer und spiritueller Natur. Das Gesamtkonzept der Palliativmedizin schließt eine Betreuung, Beratung und Anbindung der Angehörigen eines Patienten mit ein (World Health Organization 2011).

\subsubsection{Ziele von Palliativmedizin}

Eines der Kernanliegen der Palliativmedizin ist die Linderung von Symptomen (Symptomkontrolle). In der internationalen Literatur sind Schmerzen, Appetitlosigkeit, Übelkeit, Obstipation, Schwäche und Atemnot häufige Symptome von Palliativpatienten (Potter et al. 2003, Radbruch et al. 2003, Borgsteede et al. 2007).

Durch eine effektive Symptomkontrolle und die Beachtung körperlicher, psychischer, sozialer und seelsorgerischer Bedürfnisse soll die Lebensqualität des Patienten verbessert werden, so dass die letzte Zeit des Lebens in größtmöglicher Autonomie und Würde erlebt werden kann. In diesem Zusammenhang spielt eine offene, adäquate und einfühlsame Kommunikation mit dem Patienten und dessen Angehörigen eine entscheidende Rolle. Sie erstreckt sich auf den Verlauf der Erkrankung, die Phase des Sterbens und für die Angehörigen auch über den Tod des Patienten hinaus. Der Prozess des Sterbens wird als Teil des Lebens akzeptiert. Das Sterben soll weder hinausgezögert noch beschleunigt werden (World Health Organization 2011). Um den komplexen Anforderungen bei der Behandlung von Palliativpatienten gerecht zu werden, erfolgt die Betreuung durch ein multiprofessionelles Team, das sich vor allem aus Ärzten, Pflegekräften, Physiotherapeuten, Sozialarbeitern, Seelsorgern, Psychologen und Kunsttherapeuten zusammensetzt.

Ursprünglich wurde Palliativmedizin fast ausschließlich bei Patienten mit malignen Grunderkrankungen angewendet. Nur ganz vereinzelt wurden auch Patienten mit nicht-malignen Erkrankungen (z. B. amyotrophe Lateralsklerose (ALS)) behandelt. Inzwischen ist allgemein anerkannt, dass allen Patienten mit schweren unheilbaren Erkrankungen unabhängig von der Diagnose - neben Krebspatienten sind dieses v. a. Patienten mit fortgeschrittenen internistischen und 
neurologischen Erkrankungen - Palliativmedizin angeboten werden sollte (Lanken et al. 2008). Der Anteil an Nicht-Tumorpatienten in spezialisierten Palliativeinrichtungen (d. h. Palliativstationen und stationäre Hospize) in Deutschland lag im Jahr 2001 bei $5 \%$ aller behandelten Patienten (Radbruch et al. 2004).

Die Anwendung von Palliativmedizin schließt heutzutage den parallelen bzw. ergänzenden Einsatz von palliativer Therapie und damit gegebenenfalls lebensverlängernder Maßnahmen nicht aus. Im Sinne des "individualized integrated model of palliative care" sollte ein Patient sogar schon ab dem ersten Auftreten von Symptomen einer fortgeschrittenen Erkrankung mit begrenzter Lebenserwartung Zugang zu palliativmedizinischen Maßnahmen erhalten. Im Verlauf sollten diese Maßnahmen dann individuell einen schrittweise zunehmenden Anteil der Gesamttherapie ausmachen (Higginson 1993, Lanken et al. 2008).

Eine viel beachtete Studie von Temel et al. (2010) erbrachte bei Patienten mit neu diagnostiziertem nicht-kleinzelligen Bronchialkarzinom sogar einen Hinweis darauf, dass frühzeitig und routinemäßig eingesetzte palliativmedizinische Maßnahmen („Early integration“) zu einer signifikanten Lebensverlängerung führen könnten.

\subsubsection{Historie}

Eine angemessene Betreuung von Schwerstkranken und Sterbenden war im Zuge der Technisierung der Medizin und mit dem Aufkommen der Intensivmedizin vor allem nach dem Zweiten Weltkrieg zunehmend in den Hintergrund gerückt. Ausgangspunkt für die moderne Hospizbewegung und auch die Palliativmedizin war die Hospizidee, die eine Verbesserung der Versorgung dieser Patientengruppe forderte. Cicely Saunders wird als Begründerin der modernen Hospizbewegung bezeichnet; sie eröffnete 1967 mit dem St. Christopher's Hospice in London das erste Hospiz. Es hatte im Hinblick auf Zielsetzung und Organisation Modellcharakter für viele Hospiz- und Palliativeinrichtungen weltweit. Die erste Palliativstation wurde 1975 durch den Arzt Balfour Mount am Royal Victoria Hospital in Kanada gegründet. Durch seine Arbeit wurde der Begriff Palliativmedizin nachhaltig geprägt (Klaschik 2009, Radbruch et al. 1997).

In Deutschland verlief die Entwicklung der Palliativmedizin zögerlicher. Obwohl bereits in den 1960er Jahren Kontakte zwischen Deutschland und den britischen Hospizen bestanden, wurde erst 1983 die erste Palliativstation in Deutschland an der Universitätsklinik Köln eröffnet. 1985 wurde mit dem Christophorus-Hospiz-Verein in München die erste Hospizinitiative gegründet, ein Jahr später folgte das erste stationäre Hospiz (Haus Hörn) in Aachen (Klaschik 2009, Klaschik und Nauck 1998). Inzwischen gibt es in Deutschland 248 Palliativstationen und 183 stationäre Hospize (Stand 11/2011) (Wegweiser Hospiz- und Palliativmedizin Deutschland 2011). 


\subsubsection{Organisationsformen}

Palliativmedizin und Hospizbewegung verfolgen grundsätzlich die gleichen Zielsetzungen. Allerdings gibt es in Bezug auf Realisierung und Qualität große regionale Unterschiede, die auch historische Gründe haben. So entwickelten sich in Deutschland Hospize und Palliativstationen weitgehend unabhängig voneinander. Im Rahmen der Hospizbewegung wurden zahlreiche Hospize von Bürgerinitiativen, Vereinen und kirchlichen Einrichtungen begründet. Die Finanzierung erfolgte auf der Basis von Spendengeldern und die Betreuung der Patienten durch ehrenamtliche Laien. Noch heute sind Hospize von Krankenhäusern oder Pflegeeinrichtungen unabhängige Institutionen, die in der Regel pflegerisch geleitet werden. Die Finanzierung erfolgt jedoch inzwischen größtenteils über die Kranken- und Pflegekassen. Die Versorgung versteht sich v. a. als eine Begleitung der Patienten in den letzten Lebenstagen. Psychosoziale und pflegerische Aspekte stehen im Vordergrund. Die ärztliche Betreuung wird zumeist durch ambulante Konsildienste oder Hausärzte übernommen.

Palliativstationen sind dagegen aus medizinischen Einrichtungen heraus entstanden. Sie sind grundsätzlich in ein Krankenhaus integriert oder diesem angeschlossen, und die Leitung erfolgt durch einen Arzt. Auf Palliativstationen werden schwer kranke Patienten nicht nur in den letzten Lebenstagen, sondern in allen kritischen Phasen lebensbedrohlicher Erkrankungen betreut.

Seit einigen Jahren erkennen sich Hospize und Palliativstationen als sich ergänzende Institutionen, die identischen Grundsätzen folgen, an. Dies ermöglicht eine effektivere Zusammenarbeit und erleichtert die Durchsetzung politischer Ziele.

Weitere Organisationsformen neben den stationären Einrichtungen sind teilstationäre Hospizdienste („Tageshospize“) und ambulante Hospiz- und Palliativdienste (Klaschik 2009). 


\subsection{HOPE (Hospiz- und Palliativerhebung)}

Die Hospiz- und Palliativerhebung (HOPE) ist eine in Deutschland landesweit durchgeführte prospektive Erhebung zur Qualitätssicherung in der Palliativmedizin, an der Palliativstationen, Hospize, onkologische Abteilungen, Konsildienste und ambulante palliativärztliche und -pflegerische Dienste seit 1999 im jährlichen Rhythmus teilnehmen.

Sie ist das Ergebnis einer Initiative des Bundesgesundheitsministeriums von 1996, welches die Entwicklung eines einheitlichen Dokumentationssystems für Palliativpatienten für sinnvoll und notwendig erachtete. In Zusammenarbeit mit der Deutschen Gesellschaft für Palliativmedizin (DGP) und der Deutschen Krebsgesellschaft (DKG) wurde daraufhin ein Formular erarbeitet, welches die Dokumentation von personen-, krankheits- und therapiebezogenen Daten als Grundlage vorsah. Dieses Ausgangsformular von HOPE war der so genannte Basisbogen. Er wurde in den folgenden Jahren im klinischen Alltag getestet und nach den Rückmeldungen der teilnehmenden Einrichtungen mehrfach überarbeitet und erweitert. Der Basisbogen ist eine reine Fremdeinschätzung; er wird durch Ärzte, das Pflegepersonal oder ein anderes professionelles Mitglied der jeweiligen Palliativ- oder Hospizeinrichtung aus Sicht des Betreuerteams ausgefüllt.

Eine Vielzahl von Modulen, die sich spezifischen Fragestellungen und aktuellen Forschungsthemen widmen, ergänzt den Basisbogen. Ein wichtiges Modul, welches bereits seit 2000 durchgehend eingesetzt wird, ist das Modul MIDOS (Minimales Dokumentationsprogramm für Palliativpatienten) (Radbruch et al. 2000 a). MIDOS ist im Gegensatz zum Basisbogen ein Selbsteinschätzungsbogen durch den Patienten. Hier sollen vor allem das Gesamtbefinden und einzelne Symptome aus Sicht des Patienten dokumentiert werden. Um eine bessere Vergleichbarkeit der Symptomchecklisten von MIDOS und Basisbogen zu ermöglichen, wurde inzwischen eine überarbeitete Version von MIDOS, MIDOS $^{2}$, eingeführt und validiert (Stiel et al. 2010 b).

Weitere Module von HOPE befassen sich mit den Themen ethische Konflikte, Prognose und Antibiotikatherapie. 2009 wurden Module zur Thromboseprophylaxe und zur Seelsorge neu angeboten.

Die Daten werden von den Einrichtungen seit 2004 online eingegeben und durch das CLARA-Institut (Clinical Analysis, Research and Application) in Berlin zusammengeführt. Seit 1999 konnten Daten von mehr als 17.000 Palliativpatienten gesammelt werden (Radbruch et al. 2009). Das umfangreiche Datenmaterial von HOPE wurde bereits mehrfach für wissenschaftliche Studien herangezogen (Nauck et al. 2004, Radbruch et al. 2004, Krumm et al. 2008). 


\subsection{Atemnot}

\subsubsection{Definition von Atemnot}

Atemnot wird von der American Thoracic Society wie folgt definiert:

„...dyspnea is ... a subjective experience of breathing discomfort that consists of qualitatively distinct sensations that vary in intensity. The experience derives from interactions among multiple physiological, psychological, social and environmental factors, and may induce secondary physiological and behavioural responses" (American Thoracic Society 1999).

Demnach ist Atemnot ein subjektives Erleben mit erschwerter Atmung, das in seiner Ausprägung schwanken kann. Die Art der Empfindung wird von einem komplexen Zusammenspiel physiologischer, psychologischer, sozialer und umweltbedingter Faktoren beeinflusst.

Um Atemnot auszudrücken, existieren im Deutschen wie im Englischen verschiedene Begriffe wie „Kurzatmigkeit“, „Luftnot“, „erschwertes Atmen“ oder „Dyspnoe“ beziehungsweise „dyspnea“, breathlessness“, „shortness of breath“ und „difficult breathing“, die in der medizinischen Literatur synonym verwendet werden.

\subsubsection{Atemnot bei Palliativpatienten: Prävalenz und ursächliche Erkrankungen}

Atemnot ist ein häufiges Symptom von Patienten mit fortgeschrittenen unheilbaren Erkrankungen, jedoch gibt es in der Literatur unterschiedliche Angaben zur Prävalenz (Chronisch obstruktive Lungenerkrankung (COPD) 90-95 \%, Herzinsuffizienz 60-88 \%, Neoplasien 10-70 \%, Lungenkarzinom 44-69 \%, AIDS 11-62 \%) (Solano et al. 2006, Tishelman et al. 2007). Häufigkeit und Schwere von Atemnot nehmen in der Endphase einer Erkrankung zu (Conill et al. 1997, Bausewein et al. 2010 a, Currow et al. 2010). Malignompatienten leiden häufiger und stärker unter Atemnot, wenn eine pulmonale, pleurale oder mediastinale Beteiligung vorliegt (Reuben und Mor 1986).

\subsubsection{Pathophysiologie von Atemnot}

Atemnot ist ein multifaktorielles Geschehen (Cuervo Pinna et al. 2009), das nach wie vor unzureichend verstanden wird. Generiert wird sie durch ein komplexes Zusammenspiel von motorischem und sensorischem Kortex, Hirnstammneuronen, peripheren und zentralen Chemorezeptoren sowie Mechanorezeptoren in den Atemwegen und der Brustwand (Booth et al. 2008). Es gibt verschiedene Ansätze, die die Interaktionen der einzelnen Anteile zu erklären versuchen. Die am meisten anerkannte Hypothese geht davon aus, dass vom motorischen Kortex zeitgleich mit dem motorischen Kommando für die Atemmuskulatur ein Signal zum sensorischen Kortex gesandt wird, das ein Missverhältnis zwischen Angebot und Bedarf meldet und so das Gefühl Atemnot hervorruft (Manning und Schwartzstein 1995). 


\subsubsection{Einschätzung von Atemnot}

Ein Standardinstrument zur Einschätzung von Atemnot gibt es bislang nicht (Bausewein et al. 2007, Bausewein et al. 2008 b, Dorman et al. 2007). Dies erschwert den Vergleich von Patienten und Patientengruppen (Bausewein et al. 2008 b). Empfindung und Intensität von Atemnot stehen häufig in keinem engen Zusammenhang mit objektiven medizinischen Messmethoden wie Blutgasanalyse oder Lungenfunktionsuntersuchung (Heyse-Moore et al. 2000). Als Goldstandard zur Messung von Atemnot gilt demnach die Selbsteinschätzung durch den Patienten. Mehrere unterschiedliche Instrumente stehen zur Verfügung: Man unterscheidet eindimensionale (Visual Analog Scale (VAS), Numerical Rating Scale (NRS), modified Borg Scale) von multidimensionalen Instrumenten (Chronic Respiratory Disease Questionnaire (CRQ), Motor Neurone Disease Dyspnoea Rating Scale (MDRS), Cancer Dyspnoea Scale (CDS)) (Dorman et al. 2009). Letztere werden aufgrund des großen Zeitaufwandes, den sie zum Ausfüllen benötigen, hauptsächlich in der Forschung eingesetzt; für den klinischen Alltag sind sie weniger praktikabel (Bausewein et al. 2008 b).

Insbesondere in der Endphase fortgeschrittener Erkrankungen können zunehmende Schwäche und kognitive Einschränkungen die Selbsteinschätzung von Symptomen durch den Patienten unmöglich machen (Campbell et al. 2009). In einer Studie von Radbruch et al. (2000 b) zeigte sich, dass mehr als ein Drittel aller Patienten einer Palliativstation nicht in der Verfassung war, einen kurzen Fragebogen zur Lebensqualität auszufüllen. In diesen Fällen muss man regelhaft auf eine Fremdeinschätzung durch einen Angehörigen oder professionellen Betreuer ausweichen. Unklar ist, wie gut die Fremdund Selbsteinschätzung für das Symptom Atemnot übereinstimmen.

\subsubsection{Management therapierefraktärer Atemnot}

Nach Ausschöpfung aller kausalen Therapiemöglichkeiten umfasst die symptomatische Behandlung der therapierefraktären Atemnot eine Kombination aus pharmakologischen und nichtpharmakologischen Maßnahmen. In der medikamentösen Therapie konnte nur für Opioide (oral, subkutan und parenteral, nicht aber vernebelt) eine Wirksamkeit belegt werden (Jennings et al. 2001, Abernethy et al. 2003).

Obwohl häufig eingesetzt, erbringen Benzodiazepine nach derzeitiger Datenlage keinen statistisch signifikanten Nutzen zur Linderung von Atemnot (Simon et al. 2010). Auch ist ein positiver Effekt durch Sauerstoffgabe für die Mehrheit der Patienten nicht nachzuweisen (Cranston et al. 2008, Currow et al. 2009 a, Abernethy et al. 2010). Aufgrund zu geringer Daten kann über die Wirksamkeit von Phenothiazinen, vernebeltem Furosemid und Antidepressiva zum jetzigen Zeitpunkt keine Aussage getroffen werden (Simon und Bausewein 2009).

Unter den nicht-pharmakologischen Maßnahmen konnte für neuromuskuläre elektrische Stimulation, Thoraxwandvibration, Gehhilfen und Atemtraining ein positiver Effekt nachgewiesen werden (Bausewein et al. 2008 a). Für den Einsatz der nicht-invasiven Beatmung liegt bei Patienten mit COPD und ALS eine Evidenz zur Wirksamkeit vor (Heffernan et al. 2006, Kolodziej et al. 2007). 
Bezüglich Entspannungsübungen und dem Einsatz eines Handventilators ist die Datenlage nicht eindeutig, jedoch deuten erste Studien auf eine Wirksamkeit hin (Galbraith et al. 2010, Bausewein et al. 2008 a).

Insgesamt konnte bislang nur für wenige symptomatische Maßnahmen ein positiver Effekt bei der Behandlung therapierefraktärer Atemnot nachgewiesen werden. Häufig beschränkte sich die Testung dieser Maßnahmen auf bestimmte Krankheitsbilder (Malignome, COPD) (Heffernan et al. 2006, Kolodziej et al. 2007).

\subsubsection{Auswirkungen von Atemnot auf die Lebensqualität}

Atemnot ist ein sehr belastendes Symptom, das die Lebensqualität von Patienten und deren Angehörigen stark beeinträchtigt (Cuervo Pinna et al. 2009). Patienten beklagen häufig den Verlust der Fähigkeit, sich im Alltag selbst zu versorgen, verlieren das Interesse an alltäglichen Aktivitäten und leiden unter sozialer Isolation. Viele fühlen sich hilflos einer unberechenbaren Dynamik des Symptoms Atemnot ausgeliefert (Booth et al. 2003). Angst vor dem Auftreten von Atemnot und im Umgang mit dem Symptom wird oft beschrieben. Für Krebspatienten konnte ein Zusammenhang zwischen starker Ausprägung von Atemnot und einem geringen Lebenswillen gezeigt werden (Tataryn und Chochinov 2002).

Angehörige und Betreuer von Patienten mit chronischer Atemnot beschreiben in ähnlicher Weise soziale Isolation und Perspektivlosigkeit. Sie leiden häufig unter Müdigkeit, Schlaflosigkeit und Konzentrationsschwäche, vor allem nachts auch unter Angst und Hilflosigkeit (Booth et al. 2003).

\subsubsection{Assoziierte Symptome mit Atemnot}

Verschiedene Studien haben ergeben, dass Patienten mit schwerer chronischer Atemnot oft auch unter den Symptomen Depression und Angst leiden (Neuman et al. 2006, Delgado-Guay et al. 2009, American Thoracic Society 1999). Eine Behandlung von Depression und Angst scheint zu einer Reduktion einer parallel bestehenden Atemnot zu führen. Der genaue Zusammenhang zwischen den Symptomen ist jedoch unklar (Booth et al. 2008).

\subsubsection{Prognose bei Atemnot}

In der Literatur wird kontrovers diskutiert, ob das Vorhandensein und die Stärke von Atemnot zur Abschätzung der Überlebenszeit von schwer kranken Patienten herangezogen werden kann (Stiel et al. 2010 a). Für COPD- und Krebspatienten wird der Grad der Atemnot als ein möglicher prognostischer Faktor vermutet (Vigano et al. 2000, Nishimura et al. 2002). Cuervo Pinna et al. (2009) konnten keinen eindeutigen Zusammenhang zwischen Atemnot und einer geringeren Lebenserwartung nachweisen. 


\subsubsection{Was ist unklar?}

Obwohl das Symptom Atemnot viele klinische, neurophysiologische und psychologische Ähnlichkeiten zum Symptom Schmerz aufweist, ist es deutlich seltener in wissenschaftlichen Studien untersucht worden. Hauptursache hierfür ist, dass Atemnot als multifaktorielles Symptom weder im Tierversuch noch bei Freiwilligen ohne weiteres induziert werden kann. Darüber hinaus ist klinische Forschung häufig dadurch limitiert, dass Patienten mit schwerer Atemnot in der Regel so beeinträchtigt sind, dass man ihnen experimentelle Bedingungen nicht ohne weiteres zumuten kann.

In der Literatur finden sich sehr unterschiedliche Aussagen zur Prävalenz des Symptoms Atemnot bei Palliativpatienten und die Bestimmung erfolgte häufig nur in kleinen Stichproben. Zudem gibt es nur wenige Evaluationen, die Auskunft über Charakteristika (Demographie, Diagnosen, Gesamtzustand, Symptomverteilung, Therapien, Überlebenszeit) von schwer kranken Patienten mit Atemnot in einer größeren Erhebungsgruppe geben. Nur wenige Studien haben sich bislang auf Zusammenhänge und Auswirkungen anderer Symptome und Faktoren auf Atemnot konzentriert.

Obgleich bei der Einschätzung von Symptomen eine Selbsteinschätzung durch den Patienten als der Goldstandard gilt, muss gerade bei Schwerstkranken häufig auf eine Fremdeinschätzung ausgewichen werden. Bisher ist unklar, wie gut für das Symptom Atemnot die Fremdeinschätzung durch einen professionellen Betreuer mit der Selbsteinschätzung übereinstimmt.

Das Ziel der vorliegenden Arbeit ist die Bestimmung der Prävalenz von Atemnot und die Beschreibung der Charakteristika von Palliativpatienten mit Atemnot auf der Basis der Analyse einer großen Stichprobe von Palliativpatienten aus spezialisierten Palliativeinrichtungen in Deutschland. 


\subsection{Fragestellungen der vorliegenden Arbeit}

Aus dem in 1.3.9 genannten Ziel leiten sich folgende konkrete Fragestellungen ab:

1. Wie hoch ist die Prävalenz des Symptoms Atemnot bei Patienten zum Zeitpunkt der Aufnahme in eine spezialisierte stationäre Palliativeinrichtung in Deutschland?

2. Welche demographischen und klinischen Charakteristika zeigen stationäre Palliativpatienten mit Atemnot?

3. Wie unterscheiden sich stationär behandelte Palliativpatienten mit Atemnot von denen ohne Atemnot?

4. Haben Palliativpatienten mit Atemnot ein höheres Risiko, während des stationären Aufenthaltes zu versterben, als Patienten ohne Atemnot? Gibt es Unterschiede in der Überlebenszeit?

5. Lassen sich assoziierte Symptome zu Atemnot ermitteln beziehungsweise Risikofaktoren für die Entwicklung von Atemnot?

6. Wie gut stimmt für Atemnot die Fremdeinschätzung durch einen professionellen Betreuer mit der Selbsteinschätzung durch den Patienten überein? 


\section{Methodik}

\subsection{Studiendesign}

Es wurde eine Sekundäranalyse eines Registers von stationären Palliativpatienten (Hospiz- und Palliativerhebung (HOPE)) durchgeführt. Für diese Analyse wurden die Jahre 2006 bis 2008 verwendet. Schwerpunkt der Arbeit ist eine Querschnittanalyse (cross-sectional) von Patientendaten, die zum Zeitpunkt der Aufnahme in eine spezialisierte Palliativeinrichtung erhoben wurden. Für die Ermittlung der Sterbefälle und Liegezeiten wurde zusätzlich ein zweiter Dokumentationszeitpunkt (Entlassung, Tod oder Ende der Dokumentationsphase) im Sinne einer Longitudinalanalyse hinzugezogen.

Die Studie wurde der Ethikkommission der Universität Göttingen vorgelegt und in dem Schreiben vom 09.03.2009 (Antragsnummer 3/2/09, Abbildung 21 im Anhang) positiv bewertet.

\subsection{Datenerhebung von HOPE}

Die Datenerhebung von HOPE findet jedes Jahr über einen Zeitraum von jeweils 3 Monaten statt. Vor Beginn jeder neuen Umfrage werden alle Palliativeinrichtungen in Deutschland über das HOPEProjekt informiert und zur Teilnahme an der Datenerhebung eingeladen. Jede teilnehmende Einrichtung benennt eine Kontaktperson, die als Ansprechpartner fungiert und die Datenerhebung koordiniert. Pro teilnehmender Einrichtung werden zwischen dem 15. März und dem 15. Juni des jeweiligen Jahres konsekutiv bis zu 30 neu aufgenommene Patienten zu Beginn und zusätzlich bei Abschluss der Betreuung, spätestens jedoch zum Ende der Dokumentationsphase am 15. Juni, erfasst.

Bei Einsatz des Moduls MIDOS wird grundsätzlich nach vorheriger Aufklärung das Einverständnis des jeweiligen Patienten eingeholt. Für detaillierte Erläuterungen über Hintergrund und Umfang des HOPE-Projektes gibt es für Patienten und Angehörige eine Informationsbroschüre.

\subsection{Umfeld und Patienten}

Für die vorliegende Arbeit wurden aus HOPE alle Patienten der Erhebungsjahrgänge 2006 bis 2008 analysiert, die die folgenden Kriterien erfüllten (Abbildung 1):

1. Die Aufnahme der Patienten erfolgte in eine spezialisierte, stationäre Palliativeinrichtung (Palliativstation oder stationäres Hospiz).

Die Aufnahmekriterien und das Umfeld waren in diesen Institutionen im Wesentlichen einheitlich und ließen eine gute Vergleichbarkeit zu. So konnte die Analyse an einer relativ homogenen Stichprobe durchgeführt und statistische Verzerrungen (bias) reduziert werden. 
Einrichtungen und Palliativdienste, die in der Analyse keine Berücksichtigung fanden, sind onkologische Stationen, Konsildienste sowie sämtliche ambulanten Palliativdienste.

2. Die Patienten wiesen ein gültiges Aufnahmedatum vor.

Patienten mit fehlenden oder nicht plausiblen Aufnahmezeitpunkten wurden ausgeschlossen.

3. Bei den Patienten lag im Basisbogen eine Aussage zum Vorhandensein oder Fehlen des Symptoms Atemnot vor.

4. Die Dokumentation erfolgte in den Jahren 2006 bis 2008.

Die Basisbögen aus diesem Zeitraum waren weitestgehend identisch aufgebaut, was das Zusammenführen und Vergleichen der Informationen ohne größere Datenverluste ermöglichte.

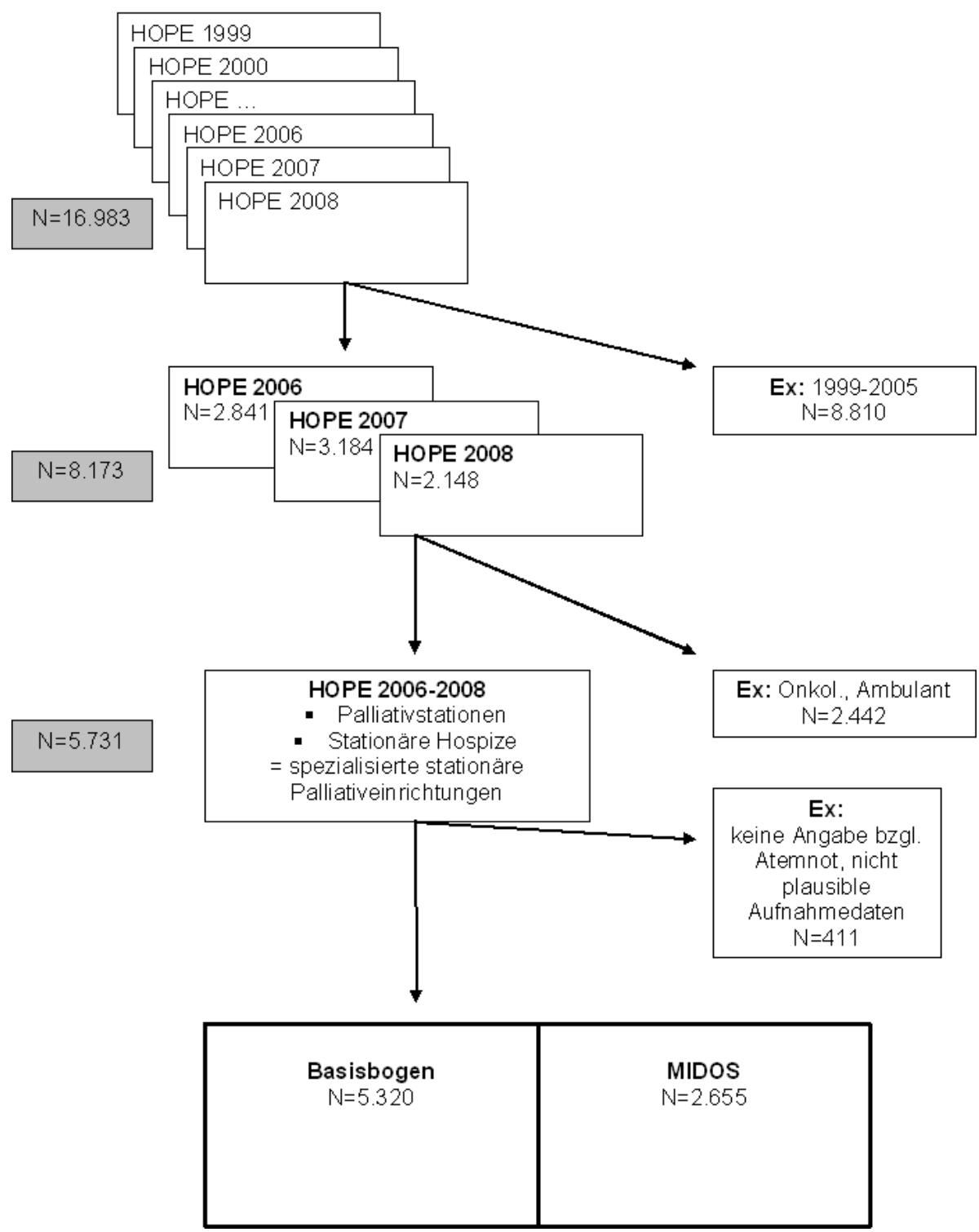

Abbildung 1: Auswahl der Patienten von HOPE für die vorliegende Arbeit

Aufgrund der beschriebenen Auswahl existieren in den Kategorien Einrichtung, Aufnahmejahr und Atemnot keine Fehldaten. 


\subsection{Instrumente}

\subsubsection{Auswahl der Dokumentationsbögen von HOPE}

Für diese Arbeit wurden von allen HOPE-Dokumentationen der Jahre 2006 bis 2008 der Basisbogen und - wenn vorhanden - der jeweils dazugehörende MIDOS-Bogen benutzt. Weitere Module von HOPE fanden in dieser Arbeit keine Berücksichtigung, da sie keinen Beitrag zur Klärung der Fragestellung leisten konnten.

\subsubsection{Basisbogen}

Der Basisbogen war die Grundlage jeder Dokumentation eines Patienten; auf ihm erfolgte die primäre Erfassung der Patientendaten. Jeder Patient erhielt zu Beginn der Dokumentation eine Identifikationsnummer (ID). So wurde eine Pseudonymisierung der Daten gewährleistet; eine eindeutige Zuordnung zu Folgebögen oder ergänzenden Modulen war jedoch immer möglich.

Auf dem Basisbogen erfolgte eine reine Fremdeinschätzung. Die Situation des Patienten wurde aus der Sicht eines professionellen Betreuers beschrieben.

Der Basisbogen (Abbildung 19 im Anhang) gliedert sich in vier Abschnitte, die zur besseren Unterscheidung im Original farblich gekennzeichnet sind. Im ersten Abschnitt (grau) wurden demographische und soziale Daten wie Alter, Geschlecht und Wohnsituation angegeben, zusätzlich das Aufnahmedatum. Der zweite Abschnitt (rot) beinhaltete Daten zum Zustand des Patienten, wie Diagnosen, Pflegestufe, Absprachen (z. B. Patientenverfügung) und Funktionsstatus. Im dritten Abschnitt (gelb) wurden weitere klinische Angaben zu Symptomen und Problemen, Medikamenten und therapeutischen Prozeduren gemacht. Der letzte Abschnitt (grün) befasste sich mit dem Betreuungsende. Hier wurden Entlass- bzw. Sterbedatum, Grund für das Ende der Therapie, Ort der Weiterbehandlung und eine abschließende Bewertung dokumentiert (Tabelle 1). 


\begin{tabular}{|l|l|}
\hline \multicolumn{2}{|c|}{ Aufbau des Basisbogens } \\
\hline 1. Abschnitt (grau) & Geburtsdatum \\
& Aufnahmedatum \\
& Geschlecht \\
& Wohnsituation \\
\hline 2. Abschnitt (rot) & Haupt- und Nebendiagnosen \\
& Datum der Erstdiagnose \\
& Metastasen \\
& Absprachen \\
& Pflegestufe \\
& Behandlungsort \\
& Funktionsstatus \\
\hline 3. Abschnitt (gelb) & Datum der Dokumentation \\
& Probleme \\
& Behandlungsziel \\
& Aktuelle Medikation \\
& Maßnahmen/ Prozeduren \\
& Welches Problem wurde besonders gut gelöst? \\
& Wer hat den Bogen ausgefüllt? \\
\hline 4. Abschnitt (grün) & Datum der Entlassung/ Tod \\
& Grund für Therapieende \\
& Weiterbehandlung durch: \\
\hline
\end{tabular}

Tabelle 1: Aufbau des Basisbogens

Bei Aufnahme des Patienten wurden die Anschnitte 1-3 ausgefüllt. Am Behandlungs- bzw. Dokumentationsende wurde zusätzlich Abschnitt 4 ausgefüllt.

Für die meisten Kategorien waren geschlossene Fragen mit vorgegebenen Antworten vorgesehen. In einigen Kategorien waren Mehrfachnennungen möglich. Ergänzend konnten Freitextangaben gemacht werden. Diagnosen wurden nach der International Classification of Diseases (ICD)-10 (Graubner 2010) kodiert oder als Freitext eingetragen. Haupt- und Nebendiagnosen wurden unterschieden. Bei Krebserkrankungen wurden vorhandene Metastasen gesondert dokumentiert. Der Funktionsstatus wurde nach der Klassifikation der Eastern Cooperative Oncology Group (ECOG) eingestuft (Tabelle 2).

\begin{tabular}{|c|c|}
\hline \multicolumn{2}{|c|}{ ECOG } \\
\hline 0 & Normale Aktivität \\
\hline 1 & gehfähig, leichte Arbeit möglich \\
\hline 2 & $\begin{array}{c}\text { nicht arbeitsfähig, kann > 50 \% der Wachzeit } \\
\text { aufstehen }\end{array}$ \\
\hline 3 & $\begin{array}{c}\text { begrenzte Selbstversorgung, > 50 \% der } \\
\text { Wachzeit bettlägerig }\end{array}$ \\
\hline 4 & pflegebedürftig, permanent bettlägerig \\
\hline
\end{tabular}

Tabelle 2: Klassifikation der Eastern Cooperative Oncology Group (ECOG) (Oken et al. 1982) 
16 unterschiedliche Symptome bzw. Probleme wurden auf einer vierstufigen kategorialen Likert-Skala (0=kein, 1=leicht, 2=mittel, 3=stark) abgefragt. Das Symptom Atemnot stand an vierter Stelle dieser Liste. Medikamente konnten in 14 Kategorien eingeordnet oder als Freitext angegeben werden. Die Dosis und der Grund für die Medikation wurden nicht erhoben. Unter den therapeutischen Prozeduren wurden nicht nur ärztliche und pflegerische Maßnahmen dokumentiert, sondern auch eine Vielzahl psychosozialer und ehrenamtlicher Leistungen.

Vermerkt wurde weiterhin, welche Profession den Basisbogen ausgefüllt hat. Krankenpflege, Arzt, Psychologe, Sozialarbeiter, Seelsorger und ehrenamtlicher Mitarbeiter standen hierfür zur Auswahl. Mehrfachnennungen waren möglich.

Am Ende des Basisbogens erfolgte eine abschließende Bewertung einmal für den Gesamtaufenthalt und ggf. zusätzlich für die Finalphase eines verstorbenen Patienten auf einer 5-stufigen kategorialen Likert-Skala (1=sehr schlecht, 2=schlecht, 3:=mittel, 4=gut, 5=sehr gut).

Trotz weitestgehender Übereinstimmung der Basisbögen der Jahrgänge 2006 bis 2008 gab es kleine Unterschiede:

- Der Basisbogen von 2006 enthielt den Unterpunkt „Woher kommt der Patient?“. Dieser wurde in den folgenden Jahren durch den Unterpunkt „Wohnsituation“ ersetzt. In der vorliegenden Arbeit wurde nur der Unterpunkt „Wohnsituation“ der Jahre 2007 und 2008 analysiert.

- In der Kategorie „Medikamente“ wurde ab 2007 der Unterpunkt „Magenschutz“ ergänzt. Für 2006 konnten Magenschutzpräparate aus dem Bereich „Sonstige Medikamente“ in eine eigene Kategorie übertragen werden.

- Die Rubrik „Prozeduren“ wurde 2007 komplett überarbeitet. Entsprechende Maßnahmen aus dem Fragebogen von 2006 wurden sinngemäß von NA und StS begutachtet und den neuen Kategorien von 2007 zugeordnet.

- Seit 2008 konnte als Grund für das Therapieende neben „Tod“ und „Entlassung“ auch „Ende der Dokumentationsphase“ angegeben werden. Für die vorliegende Analyse wurden alle Patienten mit der Angabe „Ende der Dokumentationsphase“ und „Entlassung“ als „nicht verstorben" bezeichnet.

Folgende Unterpunkte des Basisbogens wurden in der Auswertung nicht berücksichtigt:

- Krankheitsdauer (berechenbar aus Teil der Frage 10)

Begründung: Ein Erstdiagnosedatum wurde im Jahr 2006 nicht erhoben und in den Jahren 2007 und 2008 nur selten angegeben, was auf eine nicht valide und unzuverlässige Datenerhebung bei diesem Unterpunkt hindeutet.

- Nebendiagnosen (Teil von Frage 10)

Begründung: Nebendiagnosen wurden nur selten angegeben, was auf eine nicht valide und unzuverlässige Datenerhebung bei diesem Unterpunkt hindeutet. 
- Grund für die Aufnahme in die Palliativeinrichtung/ Therapieziel (Frage 15)

Begründung: Die Antworten waren häufig sehr allgemein gehalten. Beispielsweise war eine der häufigsten Antworten „Symptomkontrolle“, ohne dass ein bestimmtes Symptom angegeben wurde.

- Probleme, die am besten bzw. am schlechtesten gelöst wurden (Frage 18)

Begründung: Die Fragen wurden nur selten beantwortet, was auf eine nicht valide und unzuverlässige Datenerhebung bei diesem Unterpunkt hindeutet.

- Ort der Weiterbehandlung nach Entlassung aus der Palliativeinrichtung (Frage 22)

Begründung: Die Frage wurde nur selten beantwortet, was auf eine nicht valide und unzuverlässige Datenerhebung bei diesem Unterpunkt hindeutet.

- Abschließende Bewertung der Zufriedenheit des Teams mit der Betreuung a) für die gesamte Behandlung und b) in der Finalphase bei verstorbenen Patienten (Frage 23)

Begründung: Die Frage war nicht Ziel führend im Sinne der Fragestellung.

\subsubsection{MIDOS-Bogen}

Der MIDOS-Bogen (Abbildung 20 im Anhang) konnte in der Erhebungsphase durch die beteiligten Einrichtungen optional ergänzend zum Basisbogen eingesetzt werden und war eine Selbsteinschätzung durch den Patienten. Hier konnte der Patient Angaben über Vorhandensein und Ausprägung von körperlichen und psychischen Symptomen machen sowie seinen Gesamtzustand einschätzen. Das Symptom „Schmerz“ wurde dabei in durchschnittlichen und maximalen Schmerz untergliedert und auf einer numerischen Rangskala von 0-10 (0=kein Schmerz, 10=stärkster vorstellbarer Schmerz) angegeben. Alle weiteren Symptome wurden auf einer vierstufigen kategorialen Likert-Skala $(0=$ kein, 1=leicht, 2=mittel, 3=stark) eingestuft. Zuletzt wurde der Gesamtzustand auf einer fünfstufigen kategorialen Likert-Skala ( $1=$ sehr schlecht, $2=$ =schlecht, $3=$ mittel, $4=$ gut, $5=$ sehr gut) eingeschätzt.

Konnte eine Selbsterfassung durch den Patienten nicht stattfinden, sollte die Ursache hierfür vom jeweiligen Betreuer dokumentiert werden. Als Gründe konnten Sprachprobleme, Schwäche, kognitive Störungen, Ablehnung und Zeitmangel angegeben werden.

Der MIDOS-Bogen wurde wie der Basisbogen zunächst einmal am Aufnahmetag ausgefüllt. Eine regelmäßige Erhebung (z. B. wöchentlich) war möglich. Da die Erhebung freiwillig als zusätzliches Modul stattfand, lag nicht für alle Patienten ein MIDOS-Bogen vor.

MIDOS wurde in dieser Arbeit für einen Vergleich der Symptomeinschätzung zwischen dem Patienten und einem professionellen Betreuer herangezogen. Aus diesem Grund wurde von MIDOS nur die Symptomeinschätzung benutzt. Alle anderen Kategorien wurden nicht analysiert. Wie auch beim Basisbogen wurden nur die Daten vom Aufnahmetag berücksichtigt. 


\subsection{Analyse}

\subsubsection{Datenbereinigung}

Nach Sicherstellung einer vollständigen Pseudonymisierung der Patientendaten wurden alle Dokumentationen durch das Forschungsinstitut CLARA gesammelt und in das Statistikprogramm SPSS eingegeben. Nach Übermittlung der Daten an die Promovendin fand eine erneute Überprüfung und Bereinigung der Datensätze statt. Diese beinhaltete:

1. Nicht plausible Daten (Entlassdatum vor Aufnahmedatum, Erstdiagnosedatum vor Geburtsdatum) wurden als Fehldaten (missing data) gewertet.

2. Wurde als Entlass- oder Sterbetag ein Datum nach dem 15.06. des jeweiligen Jahres angegeben - und damit nach Beendigung der Dokumentationsphase - wurde der 15.06. als letzter Behandlungstag bestimmt und der Patient an diesem Tag als „nicht verstorben“ bezeichnet. Patienten aus dem Jahr 2008, bei denen am 15.06. „Ende der Dokumentationsphase“ angekreuzt war, wurden ebenfalls dem Kollektiv der „NichtVerstorbenen" zugeordnet.

3. Wurde als Hauptdiagnose ein Symptom oder eine Akutdiagnose (z. B. Pneumonie oder Harnwegsinfekt) kodiert, obwohl in den Nebendiagnosen eine Krebserkrankung angegeben war, wurde als Hauptdiagnose die Krebserkrankung benutzt. Sinn der Analyse der Hauptdiagnosen sollte die Bestimmung der dem Aufenthalt in einer palliativmedizinischen Einrichtung zugrunde liegenden Erkrankung sein.

4. Bei der Auswertung der Pflegestufe wurde die Rubrik „Antrag auf Pflegestufe gestellt“ nicht berücksichtigt, da sie für die Fragestellung keinen Beitrag lieferte.

\subsubsection{Statistische Datenanalyse}

Zunächst wurde die Grundgesamtheit (GG) deskriptiv beschrieben. Im Anschluss wurde die Grundgesamtheit anhand der Variablen „Atemnot“ in Patienten mit Atemnot und Patienten ohne Atemnot geteilt. So wurde auch die Prävalenz des Symptoms Atemnot in der Grundgesamtheit ermittelt.

Die beiden Untergruppen wurden dann getrennt in den einzelnen Kategorien des Basisbogens einer deskriptiven und vergleichenden Analyse unterzogen.

\section{Deskriptiver Teil:}

Für binäre, kategoriale und ordinale Daten wurden die absoluten und relativen (\%) Häufigkeiten berechnet, bei ordinalen Daten zusätzlich Median und Spannweite. Für parametrische Daten wurden Mittelwert und Standardabweichung ermittelt. 
In der Kategorie Symptome wurden für jeden Patienten Summen für die Gesamtbelastung mit Symptomen und die Schwere ihrer Ausprägung berechnet. Die Anzahl aller vorhandenen Symptome wurde als „Symptomlast“ bezeichnet und konnte Werte zwischen 0 und 12 annehmen. Die Summe aller Symptomintensitäten wurde als „Intensitätslast“ betitelt und konnte Werte zwischen 0 und 36 erreichen. Die Symptome des Basisbogens, die in die Analyse eingingen, sind Tabelle $3 \mathrm{zu}$ entnehmen. Für bestimmte Merkmale wurde die Prävalenz von Atemnot ermittelt.

\begin{tabular}{|c|}
\hline Symptome des Basisbogens \\
\hline Schmerz \\
Übelkeit \\
Erbrechen \\
Obstipation \\
Appetitlosigkeit \\
Atemnot \\
Schwäche \\
Müdigkeit \\
Angst \\
Anspannung \\
Depression \\
Desorientiertheit \\
\hline
\end{tabular}

Tabelle 3: Symptome des Basisbogens

\section{Vergleichender Teil:}

Die Gruppen der Patienten mit Atemnot und der Patienten ohne Atemnot wurden schließlich einander gegenübergestellt. Zum Vergleich der Mittelwerte wurde für binäre und kategoriale Daten der ChiQuadrat-Test, für ordinale Daten der Mann-Whitney-U-Test und für parametrische Daten der t-Test für unverbundene Stichproben angewendet. Aufgrund multipler Testungen wurde das statistische Signifikanzniveau auf $\mathrm{p}<0,01$ festgesetzt.

Zum Vergleich der Patientengruppen mit unterschiedlicher Ausprägung von Atemnot wurde die Gruppe der Patienten mit Atemnot anhand der Atemnotintensität (1=leicht; 2=mittel; 3=schwer) weiter unterteilt. Der Vergleich der Mittelwerte in diesen Untergruppen erfolgte mit dem Kruskal-WallisTest.

Um die Stärke von Zusammenhängen zwischen Atemnot und bestimmten Merkmalen beziehungsweise Symptomen anzugeben, wurden Korrelationen mit dem Spearman's-rhoKoeffizienten bestimmt. Ergebnisse von Korrelationen werden in der Literatur unterschiedlich interpretiert. Diese Arbeit stützt sich auf die Richtlinien nach Cohen (1988) (Tabelle 4).

\begin{tabular}{|c|c|}
\hline \multicolumn{2}{|c|}{ Stärke der Korrelation nach Cohen } \\
\hline $0,50-1,00$ & groß \\
\hline $0,30-0,49$ & mäßig \\
\hline $0,29-0,10$ & gering \\
\hline
\end{tabular}

Tabelle 4: Einteilung der Stärke der Korrelation nach Cohen 
Um eine mögliche Beeinflussung (prediction) von Variablen auf das Symptom Atemnot zu analysieren, wurde eine einfache (einzelne Variable; Einzelfaktorenanalyse) und multiple Regression (mehrere Variablen im Sinne eines Modells; Multiregressionsanalyse) durchgeführt und das Odds Ratio (OR) berechnet. Das Signifikanzniveau wurde hier aufgrund einer begrenzten Anzahl von Testungen auf $\mathrm{p}<0,05$ festgesetzt.

Hierbei wurden folgende Kriterien zur Auswahl der Variablen herangezogen:

1. Klinische Sinnhaftigkeit

2. Signifikanz in der Korrelationsanalyse.

Variablen, die beide Kriterien erfüllten, wurden in die Multiregressionsanalyse eingeschlossen.

Zur Güte-Bewertung der Multiregressionsanalyse wurden drei Tests verwendet:

1. Mit dem Omnibus-Test der Modelkoeffizienten wurde die Güte des Modells (Model performance) bestimmt. Ein Signifikanzwert p<0,05 gab eine hohe Güte an.

2. Der Hosmer-Lemeshow-Test diente zur Bestimmung der Passung des Modells (Goodness of fit). Ein Signifikanzwert $\mathrm{p}>0,05$ stand für eine gute Passung.

3. Der Cox-\&-Snell-R-Quadrat- und der Nagelkerke-R-Quadrat-Test wurden zur Bestimmung der Nützlichkeit des Modells (Welcher Anteil der Varianz wird durch das Modell erklärt?) herangezogen. Die Angabe erfolgte in \%. Eine Nützlichkeit wurde ab einem Prozentsatz von ca. 20-25\% oder höher angenommen (Pallant 2007).

Der Vergleich von Fremd- und Eigeneinschätzung in der Kategorie Symptome erfolgte mit dem Wert Cohen's Kappa ( $\kappa)$ als Grad der Übereinstimmung („Level of Agreement“) (Altman 1991) (Tabelle 5). Dieser wird als der wichtigste Marker zur Einschätzung der Übereinstimmung zwischen zwei Beobachtern angesehen (Stephens et al. 1997). Zusätzlich wurden für Atemnot Sensitivität und Spezifität nach folgenden Formeln berechnet:

Sensitivität $=\frac{\text { Richtig_Positive }}{(\text { Richtig_Positive }+ \text { Falsch_Negative })}$

Spezifität $=\frac{\text { Richtig_Negative }}{(\text { Richtig_Negative }+ \text { Falsch_Positive })}$ 


\begin{tabular}{|c|c|}
\hline \multicolumn{2}{|c|}{ Grad der Übereinstimmung nach Cohen's $\mathbf{K}$} \\
\hline $0,81-1,00$ & sehr gut \\
\hline $0,61-0,80$ & gut \\
\hline $0,41-0,60$ & befriedigend \\
\hline $0,21-0,40$ & ausreichend \\
\hline$<0,20$ & mangelhaft \\
\hline
\end{tabular}

Tabelle 5: Bewertung des Grads der Übereinstimmung nach Cohen's Kappa

Die statistische Datenanalyse wurde mit dem Statistikprogramm SPSS 17.0 durchgeführt. Die Auswahl der statistischen Tests erfolgte mit Hilfe der Beratung durch die Statistikerin Dr. Gao Wei des Department of Palliative Care am King's College in London, UK. 


\section{Ergebnisse}

\subsection{Beschreibung der Grundgesamtheit}

\subsubsection{Charakteristika der Grundgesamtheit}

Im Jahre 2006 nahmen 58 Palliativstationen und 22 stationäre Hospize, im Jahre 200767 bzw. 24 und im Jahre 200851 bzw. 9 Einrichtungen an der HOPE-Befragung teil. Das entspricht im Durchschnitt 40,8 \% aller Palliativstationen und 12,1\% aller stationären Hospize und damit insgesamt 26,1\% aller spezialisierten Palliativeinrichtungen in Deutschland (Tabelle 6).

\begin{tabular}{|c|c|c|c|}
\hline Jahr der Erhebung & $\begin{array}{c}\text { Gesamtzahl der } \\
\text { Einrichtungen }\end{array}$ & $\begin{array}{c}\text { Teilnehmende } \\
\text { Einrichtungen }\end{array}$ & \% \\
\hline $\begin{array}{c}\text { 2006 } \\
\text { Palliativstationen }\end{array}$ & 126 & 58 & 46,0 \\
Stationäre Hospize & 142 & 22 & 15,5 \\
\hline 2007 & 139 & 67 & 48,2 \\
Palliativstationen & 151 & 24 & 15,9 \\
Stationäre Hospize & 166 & 51 & 30,7 \\
\hline Palliativstationen & 162 & 9 & 5,6 \\
Stationäre Hospize & & & \\
\hline
\end{tabular}

Tabelle 6: Anteil der an HOPE teilnehmenden spezialisierten stationären Palliativeinrichtungen

Insgesamt gingen 5.320 Patienten in die vorliegende Analyse ein. Diese Patienten werden im Folgenden als Grundgesamtheit (GG) bezeichnet. 2006 wurden 1.861 Patienten ausgewertet, 2007 2.066 und 2008 waren es 1.393 Patienten.

\section{Demographische Daten:}

Das mittlere Alter der Patienten in der Grundgesamtheit betrug 67,2 Jahre (SD: 13,0 Jahre), 2.839 Patienten (53,4 \%) waren weiblich. Die Mehrheit der Patienten wohnte mit Angehörigen zusammen $(64,8 \%)$, knapp ein viertel $(23,5 \%)$ lebte allein und 3,9\% waren in einem Heim untergebracht. 4.228 Patienten $(79,5 \%)$ wurden auf einer Palliativstationen versorgt und 1.092 (20,5\%) in einem stationären Hospiz.

Klinische Daten:

5.014 Patienten $(94,3 \%)$ litten an einer malignen Grunderkrankung. Die drei häufigsten Krebsdiagnosen waren Lungenkarzinom (16,2 \%), Kolonkarzinom (10,8 \%) und Mammakarzinom $(10,3 \%)$. Die häufigsten Nicht-Krebsdiagnosen waren Apoplex, Anämie und Herzinsuffizienz. 
20,7 \% aller Patienten besaßen eine Patientenverfügung. Für 29,1 \% lag eine Pflegestufe vor. 74,9 \% hatten mit einem ECOG von 3 oder 4 einen niedrigen Funktionsstatus.

Verlauf:

2.024 Patienten $(38,4 \%)$ verstarben während des stationären Aufenthaltes, 1.966 (37,0 \%) überlebten (24,7 \% Fehldaten) (Tabelle 7). Von den 4.228 Patienten, die auf einer Palliativstation lagen, verstarben $34,9 \%, 44,5 \%$ überlebten. Dagegen verstarben von den 1.092 Hospizpatienten $51,8 \%$ und nur 7,7 \% überlebten (Tabelle 8).

\begin{tabular}{|c|c|c|c|c|}
\hline & GG & 2006 & 2007 & 2008 \\
\hline Anzahl (\%) & $5320(100)$ & $1861(35,0)$ & $2066(38,8)$ & $1393(26,2)$ \\
\hline $\begin{array}{l}\text { Alter } \\
\text { Mittelwert } \\
\text { SD } \\
\text { Fehldaten (\%) }\end{array}$ & $\begin{array}{c}67,2 \\
13,0 \\
116(2,2)\end{array}$ & $\begin{array}{c}67,1 \\
13,0 \\
57(3,1)\end{array}$ & $\begin{array}{c}67,3 \\
12,7 \\
47(2,3)\end{array}$ & $\begin{array}{c}67,4 \\
13,3 \\
12(0,9)\end{array}$ \\
\hline $\begin{array}{l}\text { Geschlecht (\%) } \\
\text { weiblich } \\
\text { männlich } \\
\text { Fehldaten }\end{array}$ & $\begin{array}{c}2839(53,4) \\
2420(45,5) \\
61(1,1)\end{array}$ & $\begin{array}{c}1028(55,2) \\
808(43,4) \\
25(1,3)\end{array}$ & $\begin{array}{c}1103(53,4) \\
932(45,1) \\
31(1,5)\end{array}$ & $\begin{array}{c}708(50,8) \\
680(48,8) \\
5(0,4)\end{array}$ \\
\hline $\begin{array}{l}\text { ECOG (\%) } \\
0-2 \\
3 / 4 \\
\text { Fehldaten }\end{array}$ & $\begin{array}{c}1000(18,8) \\
3986(74,9) \\
334(6,3)\end{array}$ & $\begin{array}{c}332(17,8) \\
1426(76,6) \\
103(5,5)\end{array}$ & $\begin{array}{c}36317,6) \\
1510(73,1) \\
193(9,3)\end{array}$ & $\begin{array}{c}305(21,9) \\
1050(75,4) \\
38(2,7)\end{array}$ \\
\hline $\begin{array}{l}\text { Verlauf (\%) } \\
\text { Nicht- } \\
\text { Verstorbene } \\
\text { Verstorbene } \\
\text { Fehldaten }\end{array}$ & $\begin{array}{l}1966(37,0) \\
2042(38,4) \\
1312(24,7)\end{array}$ & $\begin{array}{c}865(46,5) \\
914(49,1) \\
82(4,4)\end{array}$ & $\begin{array}{l}642(31,1) \\
686(33,2) \\
738(35,7)\end{array}$ & $\begin{array}{l}459(33,0) \\
442(31,7) \\
492(35,3)\end{array}$ \\
\hline
\end{tabular}

Tabelle 7: Demographische Daten der Grundgesamtheit und in den unterschiedlichen Erhebungsjahrgängen

\begin{tabular}{|l|c|c|c|}
\hline & GG & Palliativstation & Hospiz \\
\hline Anzahl (\%) & $5320(100)$ & $4228(79,5)$ & $1092(20,5)$ \\
\hline Verlauf (\%) & $1966(37,0)$ & $1882(44,5)$ & $84(7,7)$ \\
Nicht-Verstorbene & $2042(38,4)$ & $1476(34,9)$ & $566(51,8)$ \\
Verstorbene & $1312(24,7)$ & $870(20,6)$ & $442(40,5)$ \\
Fehldaten & & & \\
\hline
\end{tabular}

Tabelle 8: Verlauf bei den Patienten der Grundgesamtheit und in den unterschiedlichen Einrichtungen der spezialisierten stationären Palliativversorgung 


\subsubsection{Das Symptom Atemnot in der Grundgesamtheit}

Unter Atemnot litten 2.860 Patienten der Grundgesamtheit. Das entspricht einer Atemnotprävalenz von 53,8 \%. Leichte Atemnot zeigten 1.165 Patienten (40,7 \%), 936 (32,7 \%) mittlere und 759 (26,5 \%) schwere Atemnot. Die mediane Atemnotintensität aller Patienten mit Atemnot betrug 2 (mittlere Stärke) bei einer Spannweite von 1-3. Mittlere oder schwere Atemnot hatten 59,2 \% aller Atemnotpatienten.

Von allen erhobenen Symptomen in dieser Arbeit war Atemnot das 9. häufigste Symptom bei Aufnahme in die stationäre Palliativeinrichtung (Abbildung 2, Tabelle 51 im Anhang).

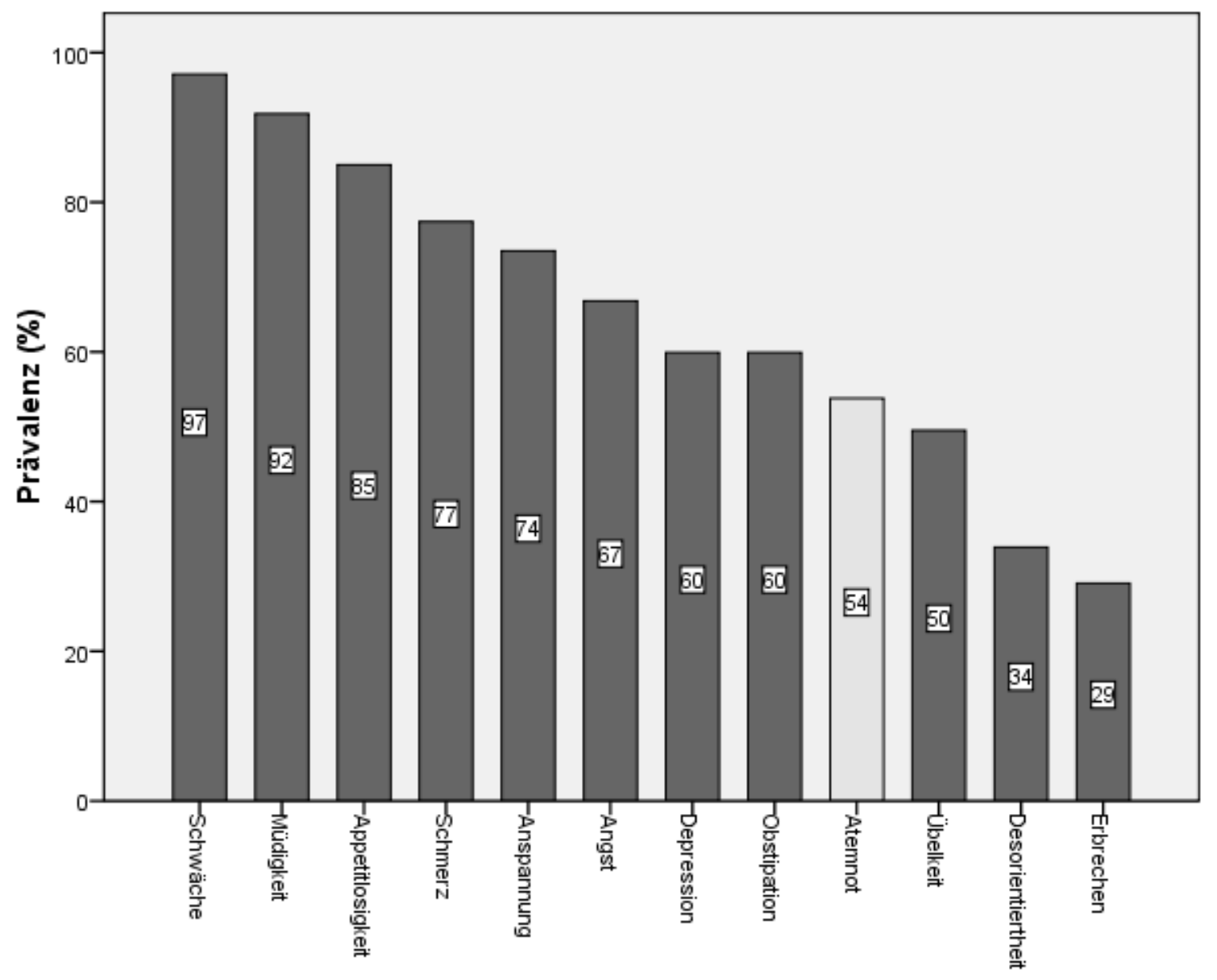

Abbildung 2: Prävalenzen der Symptome des Basisbogens absteigend nach Häufigkeit, hervorgehoben: Atemnot 


\subsection{Charakteristika von Palliativpatienten mit Atemnot}

\subsubsection{Demographische Daten}

Das mittlere Alter der Patienten mit Atemnot lag bei 67,2 Jahren (SD 12,4). 1.046 Patienten (36,6 \%) waren jünger als 65 Jahre, 190 Patienten (6,6 \%) waren 85 Jahre und älter. 1469 Patienten $(51,4 \%)$ waren weiblich, 1359 (47,5\%) männlich. Bezüglich der Wohnsituation ergab sich, dass die Mehrheit der Patienten mit Angehörigen zusammen lebte (66,0 \%), knapp ein Viertel $(22,9 \%)$ wohnte allein und 3,5\% waren in einem Heim untergebracht. Auf einer Palliativstation lagen 81,5\% aller Patienten mit Atemnot, 18,5 \% befanden sich in einem stationären Hospiz.

Verglichen mit der Patientengruppe ohne Atemnot ergaben sich keine Unterschiede im mittleren Alter. Aufgeteilt in Altersgruppen zeigte sich ein etwas jüngeres Patientenkollektiv in der Atemnotgruppe. Dieser Unterschied war jedoch nicht signifikant. Auch die Wohnsituation ergab für beide Patientengruppen ähnliche Ergebnisse. Auffällig war ein signifikant höherer Anteil von männlichen Patienten in der Atemnotgruppe (47,5\% vs. 43,1\%, p=0,001). Ebenso fand sich ein Unterschied im Vergleich der Institutionen. In der Atemnotgruppe lag ein signifikant höherer Anteil (81,5 \% vs. 77,1 $\%, \mathrm{p}<0,001$ ) auf einer Palliativstation (Tabelle 9).

\begin{tabular}{|l|l|l|l|}
\hline & Atemnot & Keine Atemnot & p \\
\hline Anzahl (\%) & $2860(53,8)$ & $2460(46,2)$ & \\
\hline Alter & & & \\
Mittelwert & 67,2 & 67,2 & 0,96 \\
$\begin{array}{l}\text { Spannweite } \\
\text { SD }\end{array}$ & $6-104$ & $6-103$ & \\
& 12,4 & 13,5 & \\
$\begin{array}{l}\text { Altersgruppen (\%) } \\
\text { < 65 }\end{array}$ & & & \\
65-74 & $1046(36,6)$ & $864(35,1)$ & 0,06 \\
$75-84$ & $915(32,0)$ & $764(31,1)$ & \\
$>84$ & $643(22,5)$ & $590(24,0)$ & \\
Fehldaten & $190(6,6)$ & $192(7,8)$ & \\
& $66(2,3)$ & $50(2,0)$ & \\
\hline $\begin{array}{l}\text { Geschlecht (\%) } \\
\text { weiblich }\end{array}$ & $1469(51,4)$ & $1370(55,7)$ & $\mathbf{0 , 0 0 1}$ \\
männlich & $1359(47,5)$ & $1061(43,1)$ & \\
Fehldaten & $32(1,1)$ & $29(1,2)$ & \\
\hline $\begin{array}{l}\text { Wohnsituation (\%) } \\
\text { (nur 2007/08, N=3459) }\end{array}$ & & & \\
& & & 0,24 \\
Allein lebend & $425(22,9)$ & $389(24,3)$ & \\
Im Heim & $65(3,5)$ & $70(4,4)$ & \\
mit Angehörigen & $1225(66,0)$ & $1015(63,3)$ & \\
Sonstige & $25(1,3)$ & $28(1,7)$ & \\
Fehldaten & $112(6,3)$ & $101(6,3)$ & \\
\hline $\begin{array}{l}\text { Institution (\%) } \\
\text { Palliativstation } \\
\text { Stationäres Hospiz }\end{array}$ & $2331(81,5)$ & $1897(77,1)$ & \\
\hline
\end{tabular}

Tabelle 9: Vergleich der demographischen Daten von Patienten mit und ohne Atemnot 
Ein entsprechendes Bild entsteht bei der Betrachtung der Atemnotprävalenzen in den einzelnen Kategorien. In den Altersgruppen zeigte sich eine sinkende Atemnotprävalenz mit zunehmendem Alter. Unter den männlichen Patienten war die Prävalenz von Atemnot höher als bei den Frauen. Die Prävalenz von Atemnot war auf Palliativstationen höher als im Hospiz (Tabelle 10).

\begin{tabular}{|l|c|}
\hline & Prävalenz von Atemnot (\%) \\
\hline Altersgruppen (Jahre) & \\
$<65$ & 54,8 \\
$65-74$ & 54,5 \\
$75-84$ & 52,2 \\
$>84$ & 49,7 \\
& \\
Fehldaten (2,2 \%) & 56,9 \\
\hline Geschlecht & \\
weiblich & 51,7 \\
männlich & 56,2 \\
Fehldaten (1,1 \%) & 52,5 \\
\hline Institution & \\
Palliativstation & 55,1 \\
Stat. Hospiz & 48,4 \\
\hline
\end{tabular}

Tabelle 10: Prävalenz von Atemnot in Abhängigkeit von Alter, Geschlecht und Institution

\subsubsection{Klinische Daten}

\subsubsection{Hauptdiagnosen}

\subsection{Patienten mit Krebserkrankung}

Ein Malignom als Hauptdiagnose ergab sich für 93,6 \% aller Patienten mit Atemnot. Krebserkrankungen zeigten sich etwas häufiger in der Nicht-Atemnotgruppe (95,0 \% vs. 93,6 \%). Der Unterschied war jedoch nicht signifikant (Tabelle 11).

\begin{tabular}{|l|l|l|l|}
\hline & Atemnot & Keine Atemnot & p \\
\hline Krebsdiagnose (\%) & $2676(93,6)$ & $2338(95,0)$ & 0,03 \\
Ja & $173(6,0)$ & $116(4,7)$ & \\
Nein & $11(0,4)$ & $6(0,2)$ & \\
Fehldaten & & & \\
\hline
\end{tabular}

Tabelle 11: Häufigkeit von Krebsdiagnosen bei Patienten mit und ohne Atemnot

Entsprechend ergab sich eine etwas geringere Atemnotprävalenz in der Gruppe der Malignompatienten (Tabelle 12). 


\begin{tabular}{|l|c|}
\hline & Prävalenz von Atemnot (\%) \\
\hline Krebsdiagnose & 53,4 \\
Ja & 59,9 \\
Nein & 64,7 \\
Fehldaten $(0,3 \%)$ & \\
\hline
\end{tabular}

Tabelle 12: Atemnotprävalenz in Abhängigkeit von einer malignen Erkrankung

Patienten mit Krebserkrankungen zeigten insgesamt eine geringere Atemnotintensität als Patienten ohne Krebserkrankung. Der Unterschied war jedoch nicht signifikant (Tabelle 13, Abbildung 3).

\begin{tabular}{|l|l|l|l|}
\hline & $\begin{array}{l}\text { Patienten mit } \\
\text { Krebserkrankung }\end{array}$ & $\begin{array}{l}\text { Patienten ohne } \\
\text { Krebserkrankung }\end{array}$ & $\mathbf{p}$ \\
\hline $\begin{array}{l}\text { Atemnotintensität } \\
(\%)\end{array}$ & & $116(40,1)$ & \\
Keine & $2338(46,6)$ & $65(22,5)$ & 0,02 \\
Leicht & $1096(21,9)$ & $59(20,4)$ & \\
Mittel & $875(17,5)$ & $49(17,0)$ & \\
Stark & $705(14,1)$ & & \\
\hline
\end{tabular}

Tabelle 13: Atemnotintensitäten bei Patienten mit und ohne Krebserkrankung

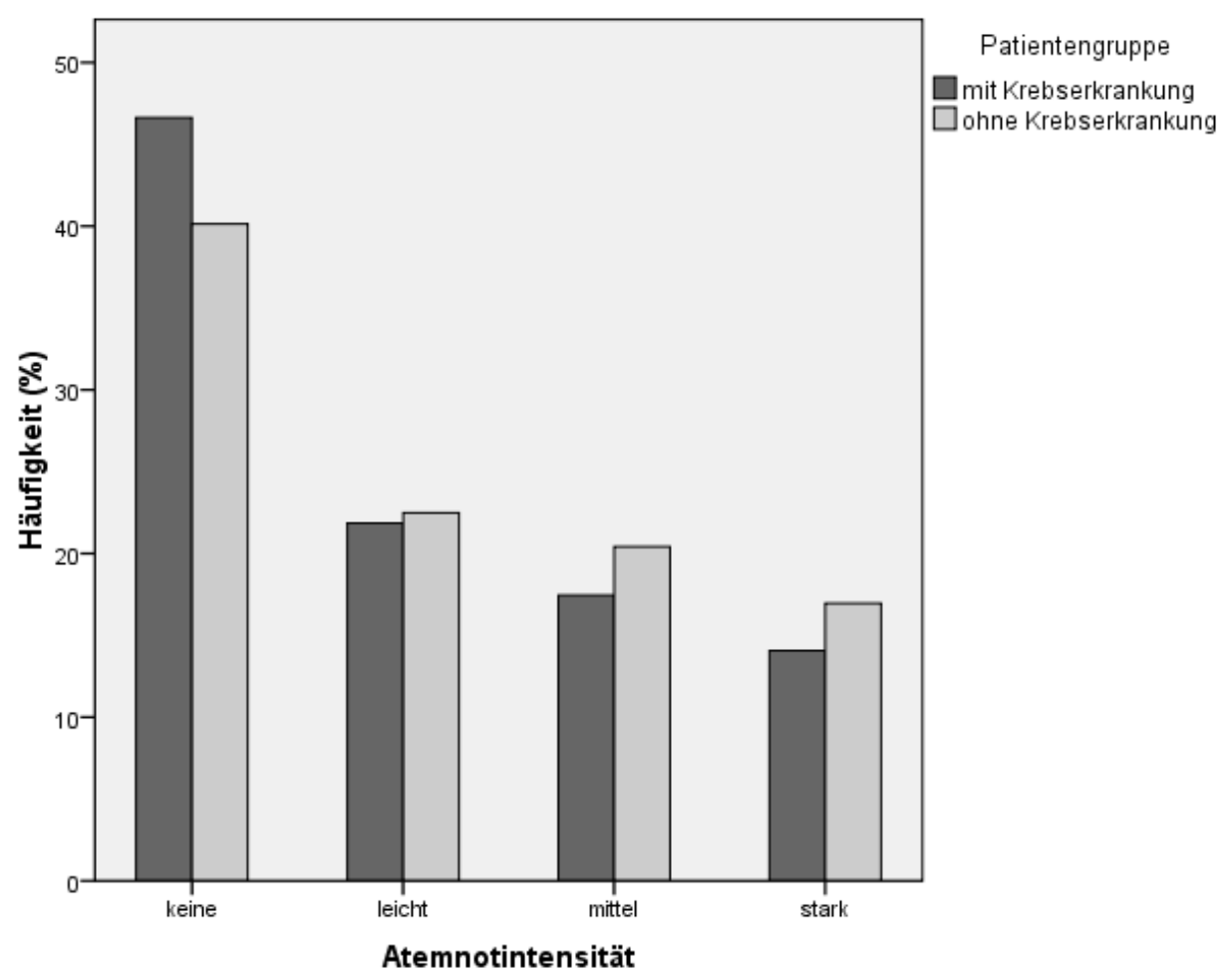

Abbildung 3: Häufigkeit unterschiedlicher Atemnotintensitäten in Abhängigkeit vom Vorhandensein einer Krebserkrankung 
Die häufigsten Krebsdiagnosen von Patienten mit Atemnot waren Bronchial- (22,4 \%), Mamma- (10,6 $\%)$ und kolorektales Karzinom (9,5\%) (Tabelle 14).

\begin{tabular}{|c|c|}
\hline \multicolumn{2}{|c|}{ Die häufigsten Krebsdiagnosen bei Patienten mit und ohne Atemnot (\%) } \\
\hline $\begin{array}{l}\text { Patienten mit Atemnot } \\
\mathrm{N}=2860\end{array}$ & $\begin{array}{l}\text { Patienten ohne Atemnot } \\
\mathrm{N}=2460\end{array}$ \\
\hline 1. Bronchialkarzinom $642(22,4)$ & 1. kolorektales Karzinom $304(12,4)$ \\
\hline 2. Mammakarzinom $304(10,6)$ & 2. Mammakarzinom $245(10,0)$ \\
\hline 3. kolorektales Karzinom $271(9,5)$ & 3. Bronchialkarzinom $222(9,0)$ \\
\hline 4. Pankreaskarzinom $145(5,1)$ & 4. Pankreaskarzinom $177(7,2)$ \\
\hline 5. Ovarialkarzinom $103(3,6)$ & 5. Prostatakarzinom $134(5,4)$ \\
\hline 6. Prostatakarzinom $101(3,5)$ & 6. Magenkarzinom $119(4,8)$ \\
\hline 7. Karzinom des MRR $89(3,1)$ & 7. Hirntumor $118(4,8)$ \\
\hline 8. Magenkarzinom $86(3,0)$ & 8. Karzinom des LGS $92(3,7)$ \\
\hline 9. Karzinom des LGS $83(2,9)$ & 9. Ovarialkarzinom $89(3,6)$ \\
\hline 10. Nierenkarzinom $68(2,4)$ & 10. Uteruskarzinom $84(3,4)$ \\
\hline
\end{tabular}

Tabelle 14: Die häufigsten Krebsdiagnosen bei Patienten mit und ohne Atemnot

Bei weiblichen Patienten mit Atemnot waren die drei häufigsten Krebsdiagnosen Mamma- (20,3 \%), Bronchial- (15,7 \%) und kolorektales Karzinom (10,3\%), männliche Patienten mit Atemnot litten v. a. an Bronchial- $(29,5 \%)$, kolorektalem $(8,7 \%)$ und Prostatakarzinom $(7,4 \%)$ (Tabelle 15).

\begin{tabular}{|c|c|}
\hline \multicolumn{2}{|c|}{ Die häufigsten Krebsdiagnosen bei Frauen und Männern mit Atemnot (\%) } \\
\hline $\begin{array}{c}\text { Frauen } \\
\mathrm{N}=1469\end{array}$ & $\begin{array}{l}\text { Männer } \\
\mathrm{N}=1359\end{array}$ \\
\hline 1. Mammakarzinom $298(20,3)$ & 1. Bronchialkarzinom $401(29,5)$ \\
\hline 2. Bronchialkarzinom $231(15,7)$ & 2. kolorektales Karzinom $118(8,7)$ \\
\hline 3. kolorektales Karzinom $151(10,3)$ & 3. Prostatakarzinom $100(7,4)$ \\
\hline 4. Ovarialkarzinom $103(7,0)$ & 4. Karzinom des MRR $69(5,1)$ \\
\hline 5. Pankreaskarzinom $79(5,4)$ & 5. Pankreaskarzinom $66(4,9)$ \\
\hline 6. Uteruskarzinom $47(3,2)$ & 6. Ösophaguskarzinom $54(4,0)$ \\
\hline 7. Magenkarzinom $40(2,7)$ & 7. Karzinom des LGS $45(3,3)$ \\
\hline 8. Karzinom des LGS $36(2,5)$ & 7. Magenkarzinom $45(3,3)$ \\
\hline 9. Lymphom $32(2,2)$ & 9. Nierenkarzinom $44(3,2)$ \\
\hline 10. Hautkrebs $28(1,9)$ & 10. Harnblasenkarzinom $35(2,6)$ \\
\hline
\end{tabular}

Tabelle 15: Die häufigsten Krebsdiagnosen bei Patienten mit Atemnot in Abhängigkeit vom Geschlecht 
Bronchialkarzinome fanden sich signifikant häufiger in der Atemnotgruppe $(22,4 \%$ vs. 9,0\%, p <0,001). Deutlich mehr Männer (29,5\%) als Frauen (15,7\%) mit Atemnot hatten als Hauptdiagnose ein Bronchialkarzinom. Der Unterschied zur Nicht-Atemnotgruppe war aber für beide Geschlechter signifikant $(\mathrm{p}<0,001)$ (Tabelle 16).

\begin{tabular}{|l|l|l|l|}
\hline & Atemnot & Keine Atemnot & $\mathbf{p}$ \\
\hline $\begin{array}{l}\text { Bronchialkarzinom } \\
\text { (\%) }\end{array}$ & & & \\
Ja & $642(22,4)$ & $222(9,0)$ & $<\mathbf{0 0 1}$ \\
Nein & $1904(66,6)$ & $2005(81,5)$ & \\
Fehldaten & $314(11,0)$ & $233(9,5)$ & \\
- bei Frauen & & & \\
(N=2839) & & $93(6,8)$ & \\
Ja & $231(15,7)$ & $1142(83,4)$ & \\
Nein & $1086(73,5)$ & $135(9,9)$ & \\
Fehldaten & $152(10,3)$ & & \\
- bei Männern & & & \\
(N=2420) & & $126(11,9)$ & $<\mathbf{0 , 0 0 1}$ \\
Ja & $401(29,5)$ & $841(79,3)$ & \\
Nein & $805(59,2)$ & $94(8,9)$ & \\
Fehldaten & $153(11,3)$ & & \\
\hline
\end{tabular}

Tabelle 16: Häufigkeit des Bronchialkarzinoms bei Patienten mit und ohne Atemnot, auch in Abhängigkeit vom Geschlecht

Entsprechend zeigte sich mit 74,3 \% vs. 48,7 \% eine höhere Atemnotprävalenz bei Patienten mit einem Bronchialkarzinom als bei Patienten ohne ein Bronchialkarzinom. Innerhalb der Bronchialkarzinomgruppe war die Atemnotprävalenz bei den Männern höher als bei den Frauen (Tabelle 17).

\begin{tabular}{|l|c|}
\hline & Prävalenz von Atemnot (\%) \\
\hline Bronchialkarzinom & $\mathbf{7 4 , 3}$ \\
Frauen & 71,3 \\
Männer & 76,1 \\
& \\
Ohne Bronchialkarzinom & $\mathbf{4 8 , 7}$ \\
Frauen & 48,7 \\
Männer & 48,9 \\
\hline
\end{tabular}

Tabelle 17: Geschlechtsspezifische Atemnotprävalenzen in Abhängigkeit vom Vorliegen eines Bronchialkarzinoms 
Neben dem Bronchialkarzinom fand sich für keine weitere Tumorentität ein signifikant höherer Anteil in der Atemnotgruppe. Jedoch zeigten das kolorektale Karzinom, das Pankreaskarzinom, das Prostatakarzinom sowie das Magenkarzinom ein signifikant selteneres Auftreten in der Atemnotgruppe (Abbildung 4, Tabelle 52 im Anhang).

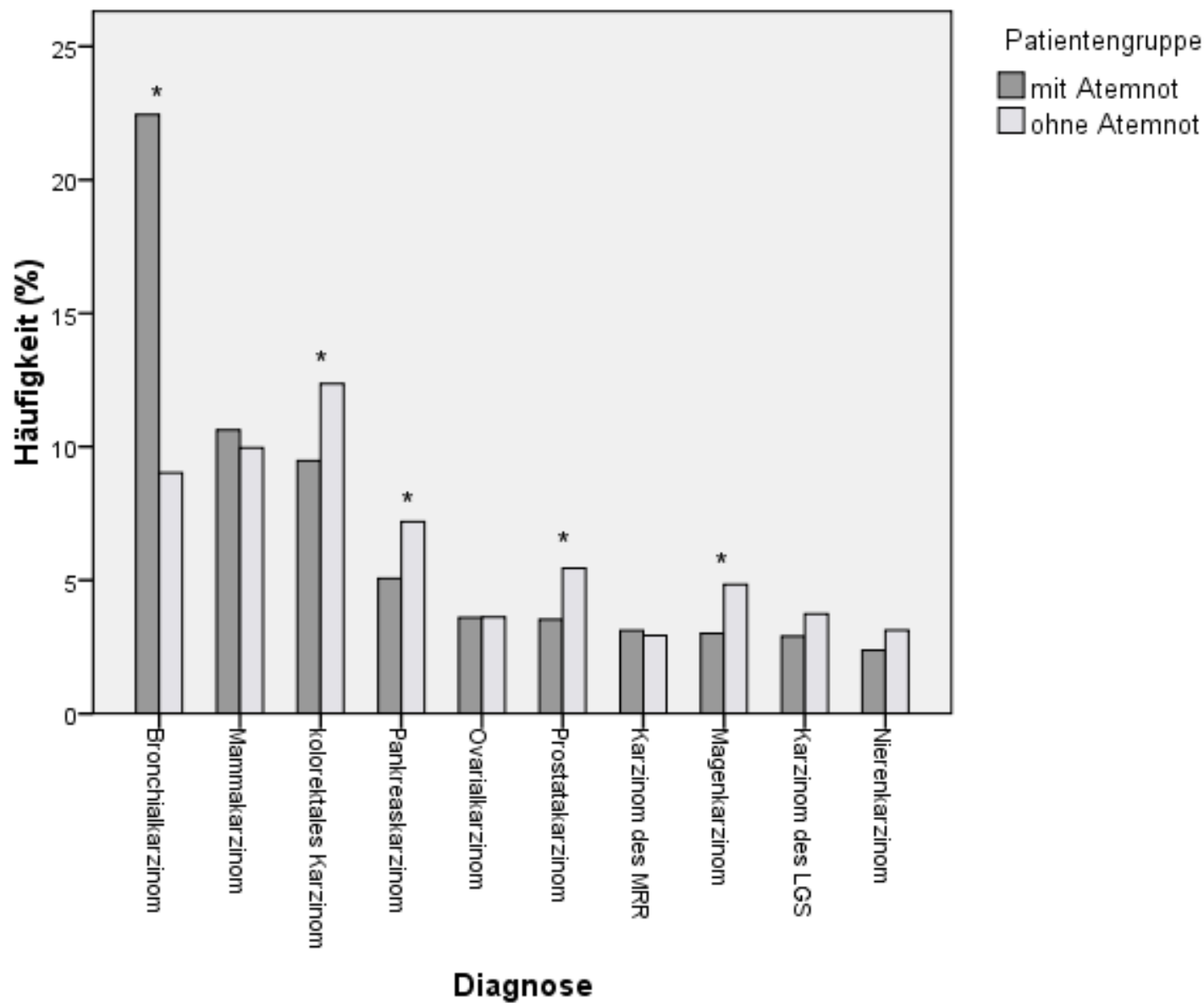

Abbildung 4: Vorkommen der 10 häufigsten Krebserkrankungen von Patienten mit Atemnot bei Patienten mit und ohne Atemnot (* signifikanter Unterschied) 
Die Atemnotprävalenzen der 10 häufigsten Tumorentitäten von Patienten mit Atemnot sind Tabelle 18 zu entnehmen.

\begin{tabular}{|l|c|}
\hline Krebsdiagnose & Prävalenz von Atemnot (\%) \\
\hline 1. Bronchialkarzinom & 74,3 \\
2. Mammakarzinom & 55,4 \\
3. Karzinom des MRR & 55,3 \\
4. Ovarialkarzinom & 53,6 \\
5. Karzinom des LGS & 47,4 \\
6. kolorektales Karzinom & 47,1 \\
7. Nierenkarzinom & 46,9 \\
8. Pankreaskarzinom & 45,0 \\
9. Prostatakarzinom & 43,0 \\
10. Magenkarzinom & 42,0 \\
\hline
\end{tabular}

Tabelle 18: Atemnotprävalenzen bei den 10 häufigsten Tumorentitäten von Patienten mit Atemnot

Die Anteile der Malignompatienten mit zusätzlich pulmonalen oder pleuralen Metastasen finden sich in Tabelle 19. Dabei zeigte sich, dass Patienten mit Bronchial-, Nieren- und Mammakarzinom deutlich häufiger an pulmonalen oder pleuralen Metastasen litten als Patienten mit Pankreas-, Magenoder Prostatakarzinom. Für Patienten mit kolorektalem Karzinom ergab sich eine leicht über dem Durchschnitt aller Malignompatienten - diese betrug 36,6 \% - liegende Häufigkeit.

\begin{tabular}{|l|c|c|}
\hline \multicolumn{1}{|c|}{ Tumorentität } & Anzahl & $\begin{array}{c}\text { Anteil mit pulmonalen oder } \\
\text { pleuralen Metastasen (\%) }\end{array}$ \\
\hline Alle Malignome & 5014 & 36,6 \\
\hline & & 61,2 \\
\hline Bronchialkarzinom & 864 & 57,2 \\
\hline Nierenkarzinom & 145 & 48,6 \\
\hline Mammakarzinom & 549 & 39,5 \\
\hline kolorektales Karzinom & 575 & 21,7 \\
\hline Karzinom des MRR & 161 & 21,1 \\
\hline Karzinom des LGS & 175 & 20,8 \\
\hline Ovarialkarzinom & 192 & 20,5 \\
\hline Pankreaskarzinom & 322 & 20,0 \\
\hline Magenkarzinom & 205 & 14,9 \\
\hline Prostatakarzinom & 235 & $\mathrm{~d}$ \\
\hline
\end{tabular}

Tabelle 19: Anteil der Patienten mit pulmonalen oder pleuralen Metastasen bei den 10 häufigsten Tumorentitäten von Patienten mit Atemnot 
Sowohl für die Gruppe der Krebspatienten mit pulmonalen oder pleuralen Metastasen als auch für die ohne ergab sich ein höherer Anteil von Patienten mit einem kolorektalen Karzinom in der NichtAtemnot-Gruppe. Der Unterschied war aber nur für die Gruppe der Krebspatienten mit pulmonalen oder pleuralen Metastasen signifikant (Tabelle 20).

\begin{tabular}{|l|l|l|l|}
\hline $\begin{array}{l}\text { Nur Krebspatienten } \\
\text { N=5014 }\end{array}$ & Atemnot & Keine Atemnot & $\mathbf{p}$ \\
\hline kolorektales & & & \\
Karzinom (\%) & $\mathrm{N}=2676$ & $\mathrm{~N}=2338$ & \\
Ja & $271(10,1)$ & $304(13,0)$ & $\mathbf{0 , 0 0 3}$ \\
Nein & $2102(78,6)$ & $1807(77,3)$ & \\
Fehldaten & $303(11,3)$ & $227(9,7)$ & \\
- mit Lungen- oder & & & \\
Pleurametastasen & & & \\
N=1834 & $\mathrm{N}=1243$ & $\mathrm{~N}=591$ & \\
Ja & $129(10,4)$ & $98(16,6)$ & \\
Nein & $980(78,8)$ & $441(74,6)$ & \\
Fehldaten & $134(10,8)$ & $52(8,8)$ & \\
-ohne Lungen- oder & & & \\
Pleurametastasen & & & \\
N=3180 & $\mathrm{N}=1433$ & $\mathrm{~N}=1747$ & \\
Ja & $142(9,9)$ & $206(11,8)$ & 0,13 \\
Nein & $1122(78,3)$ & $1366(78,2)$ & \\
Fehldaten & $169(11,8)$ & $175(10,0)$ & \\
& & & \\
\hline
\end{tabular}

Tabelle 20: Häufigkeit des kolorektalen Karzinoms bei Krebspatienten mit und ohne Atemnot in Abhängigkeit vom Vorhandensein pulmonaler oder pleuraler Metastasen

Entsprechend zeigte sich für Patienten mit einem kolorektalen Karzinom ohne pulmonale oder pleurale Metastasen eine niedrigere, bei Patienten mit kolorektalem Karzinom mit pulmonalen oder pleuralen Metastasen sogar eine deutlich niedrigere Atemnotprävalenz als bei Patienten mit einem anderen Malignom.

\begin{tabular}{|l|c|}
\hline & Prävalenz von Atemnot (\%) \\
\hline Lungen- oder Pleurametastasen & $\mathbf{6 7 , 8}$ \\
- bei kolorektalem Karzinom & 56,8 \\
- bei anderem Malignom & 69,0 \\
& \\
Ohne Lungen- oder & $\mathbf{4 5 , 1}$ \\
Pleurametastasen & 40,8 \\
- bei kolorektalem Karzinom & 45,1 \\
\hline - bei anderem Malignom & \\
\hline
\end{tabular}

Tabelle 21: Atemnotprävalenzen bei Krebspatienten mit und ohne Lungen- oder Pleurametastasen, auch in Abhängigkeit vom Vorliegen eines kolorektalen Karzinoms 
Metastasen:

Metastasen ergaben sich bei $88,9 \%$ aller Krebspatienten mit Atemnot. Am häufigsten zeigten sich Lungen- (42,5\%), Leber- (35,3\%) und Knochenmetastasen (32,2 \%).

Demgegenüber hatten Krebspatienten ohne Atemnot mit 83,2 \% signifikant seltener Metastasen $(\mathrm{p}<0,001)$. Im Vergleich hatten Krebspatienten mit Atemnot signifikant häufiger Lungen-, Pleura- und Lymphknotenmetastasen als Patienten ohne Atemnot (Tabelle 22, Abbildung 5).

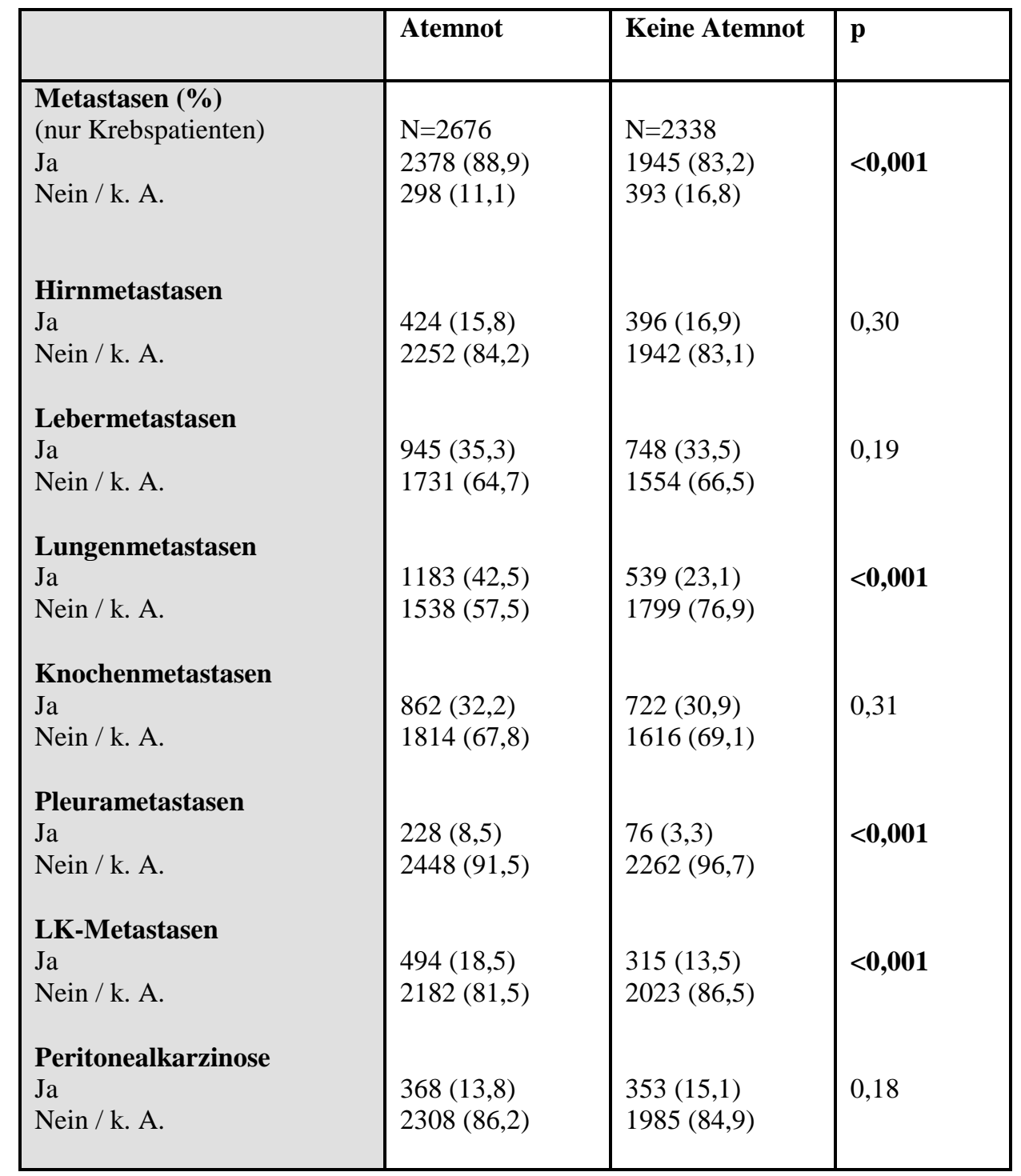

Tabelle 22: Häufigkeit von Metastasen bei Krebspatienten mit und ohne Atemnot 


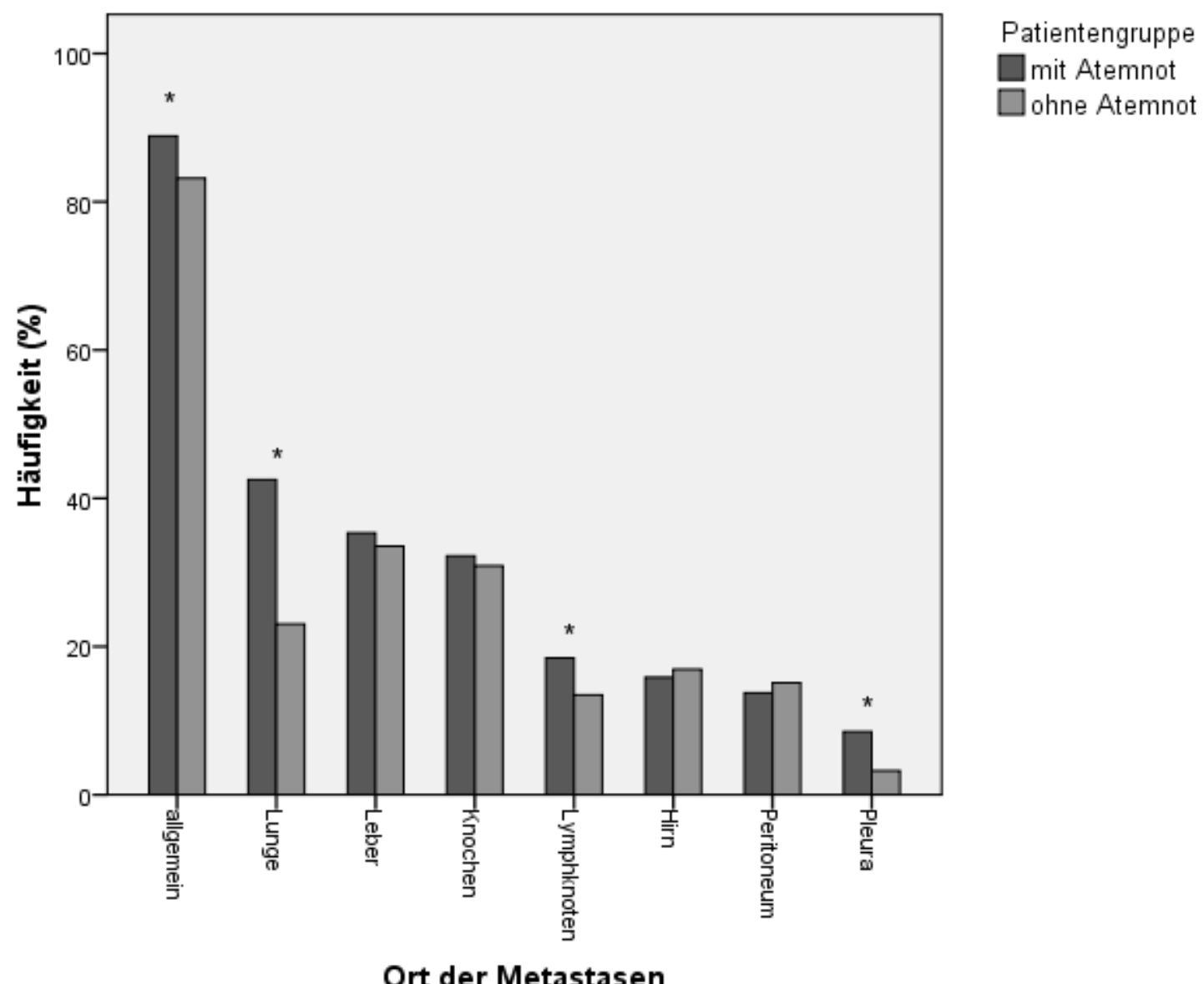

Abbildung 5: Häufigkeit von Metastasen bei Patienten mit und ohne Atemnot (*: signifikanter Unterschied)

Die Atemnotprävalenzen in den Patientengruppen mit und ohne Metastasen sowie bei unterschiedlichen Metastasenlokalisationen sind Tabelle 23 zu entnehmen.

\begin{tabular}{|l|c|}
\hline & Prävalenz von Atemnot (\%) \\
\hline Metastasen & 55,0 \\
Ja & 48,3 \\
Nein & \\
\hline Metastasenlokalisation & \\
1. Pleura & 75,2 \\
2. Lunge & 67,9 \\
3. Lymphknoten & 61,1 \\
5. Leber & 54,7 \\
6. Knochen & 54,4 \\
7. Hirn & 51,0 \\
8. Peritoneum & 51,7 \\
\end{tabular}

Tabelle 23: Atemnotprävalenzen in Abhängigkeit vom Vorhandensein bzw. der Lokalisation von Metastasen 
Bei Patienten mit bösartiger primärer oder sekundärer Neubildung in der Lunge war die Atemnotprävalenz ebenfalls deutlich erhöht (Tabelle 24).

\begin{tabular}{|l|c|}
\hline & Prävalenz von Atemnot (\%) \\
\hline Bösartige Neubildung der Lunge & 68,0 \\
Ja & 44,0 \\
Nein & \\
\hline
\end{tabular}

Tabelle 24: Atemnotprävalenzen in Abhängigkeit vom Vorhandensein einer bösartigen Neubildung in der Lunge

\subsection{Patienten ohne Krebserkrankung}

Keine Tumorerkrankung als Hauptdiagnose hatten 6,0 \% aller Patienten mit Atemnot. Die häufigsten Diagnosen waren cerebraler Insult (12,7\%), Chronisch Obstruktive Lungenerkrankung (COPD, 11,6 $\%$ ) und Herzinsuffizienz (CHF, 10,4\%) (Tabelle 25).

\begin{tabular}{|lc|}
\hline \multicolumn{1}{|c|}{$\begin{array}{c}\text { Die 10 häufigsten Nicht-Krebsdiagnosen } \\
\text { von Patienten mit Atemnot }(\%) \\
\text { N=173 }\end{array}$} \\
\hline 1. Cerebraler Insult & $22(12,7)$ \\
2. COPD & $20(11,6)$ \\
3. Chronische Herzinsuffizienz & $18(10,4)$ \\
4. Anämie & $15(8,7)$ \\
5. Multisystematrophie & $15(8,7)$ \\
6. Leberzirrhose & $9(5,2)$ \\
7. KHK & $8(4,6)$ \\
8. Niereninsuffizienz & $7(4,0)$ \\
9. chronisches Schmerzsyndrom & $6(3,5)$ \\
10. Demenz & $5(2,9)$ \\
\hline
\end{tabular}

Tabelle 25: Die häufigsten Nicht-Krebs-Diagnosen von Patienten mit Atemnot

Patienten ohne Atemnot hatten in 4,7\% der Fälle eine Nicht-Tumorerkrankung. Sowohl eine COPD $(11,6 \%$ vs. $1,7 \%)$ als auch eine Herzinsuffizienz (10,4\% vs. 5,2 \%) wurden häufiger im Atemnotkollektiv gefunden, signifikant war der Unterscheid jedoch nur für die COPD $(p=0,002)$ (Tabelle 26). 


\begin{tabular}{|l|l|l|l|}
\hline & Atemnot & Keine Atemnot & p \\
\hline Anzahl & 173 & 116 & \\
\hline $\begin{array}{l}\text { COPD (\%) } \\
\text { Ja } \\
\text { Nein }\end{array}$ & $\begin{array}{l}20(11,6) \\
153(88,4)\end{array}$ & $\begin{array}{l}2(1,7) \\
114(98,3)\end{array}$ & $\mathbf{0 , 0 0 2}$ \\
\hline $\begin{array}{l}\text { Herzinsuffizienz(\%) } \\
\text { Ja } \\
\text { Nein }\end{array}$ & $18(10,4)$ & $\begin{array}{l}6(5,2) \\
110(94,8)\end{array}$ & 0,11 \\
\hline
\end{tabular}

Tabelle 26: Häufigkeit von COPD und chronischer Herzinsuffizienz bei Patienten ohne Krebserkrankung mit und ohne Atemnot

Sowohl Patienten mit COPD als auch Patienten mit chronischer Herzinsuffizienz hatten eine hohe Atemnotprävalenz (Tabelle 27).

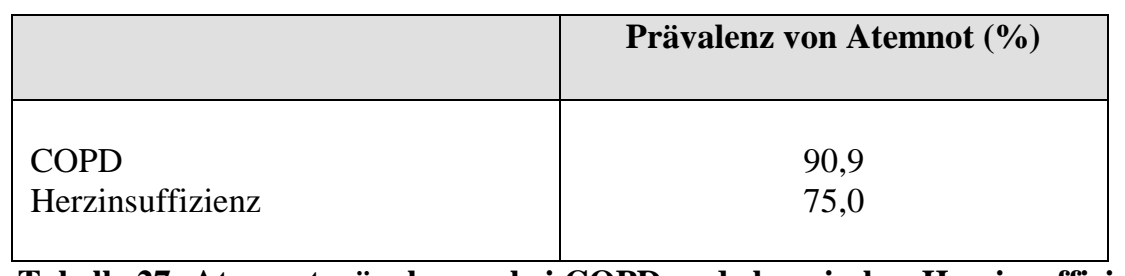

Tabelle 27: Atemnotprävalenzen bei COPD und chronischer Herzinsuffizienz

Für 52,8 \% aller Patienten mit Atemnot konnte entweder eine maligne Erkrankung der Lunge oder eine sonstige chronische pulmonale Vorerkrankung wie Herzinsuffizienz oder COPD gefunden werden. Umgekehrt fehlt dieser Bezug für 47,2 \% aller Patienten mit Atemnot. Lungenerkrankungen fanden sich signifikant häufiger bei Patienten mit Atemnot als bei Patienten ohne Atemnot (52,8\% vs. $28,5 \%, \mathrm{p}<0,001$ ) (Tabelle 28).

\begin{tabular}{|l|l|l|l|}
\hline & Atemnot & Keine Atemnot & $\mathbf{p}$ \\
\hline $\begin{array}{l}\text { Lungenerkrankung } \\
\left(\begin{array}{l}\text { \% }) \\
\text { Ja } \\
\text { Nein }\end{array}\right.\end{array}$ & $1511(52,8)$ & $702(28,5)$ & \\
& $1349(47,2)$ & $1758(71,5)$ & $<\mathbf{0 , 0 0 1}$ \\
\hline
\end{tabular}

Tabelle 28: Häufigkeit von Lungenerkrankungen bei Patienten mit und ohne Atemnot. 


\subsubsection{Funktionsstatus}

Der Funktionsstatus (ECOG 0-4) von Patienten mit Atemnot im Vergleich zu Patienten ohne Atemnot ist Tabelle 29 und Abbildung 6 zu entnehmen. Auch wenn der mediane ECOG in beiden Gruppen bei 3 lag, zeigten die Patienten mit Atemnot einen signifikant schlechteren Funktionsstatus als Patienten ohne Atemnot ( $\mathrm{p}<0,001) .78,4 \%$ aller Patienten mit Atemnot hatten einen niedrigen Funktionsstatus (ECOG 3 oder 4), dies galt jedoch nur für 70,8\% aller Patienten ohne Atemnot ( $<<0,001)$.

\begin{tabular}{|l|l|l|l|}
\hline & Atemnot & Keine Atemnot & $\mathbf{p}$ \\
\hline $\begin{array}{l}\text { Funktionsstatus } \\
(\boldsymbol{\%})\end{array}$ & & & \\
0 & $5(0,2)$ & $15(0,6)$ & $<\mathbf{0 , 0 0 1}$ \\
1 & $76(2,7)$ & $122(5,0)$ & \\
2 & $357(12,5)$ & $425(17,3)$ & \\
3 & $973(34,0)$ & $786(31,2)$ & \\
4 & $1271(44,4)$ & $956(38,9)$ & \\
Fehldaten & $178(6,2)$ & $156(6,3)$ & \\
& & & \\
Median & 3 & 3 & \\
\hline Funktionsstatus- & & & \\
Gruppen (\%) & $438(15,4)$ & $562(22,9)$ & \\
$0-2$ & $2244(78,4)$ & $1742(70,8)$ & \\
3 oder 4 & $178(6,2)$ & $156(6,3)$ & \\
Fehldaten & & & \\
\hline
\end{tabular}

Tabelle 29: Funktionsstatus von Patienten mit und ohne Atemnot

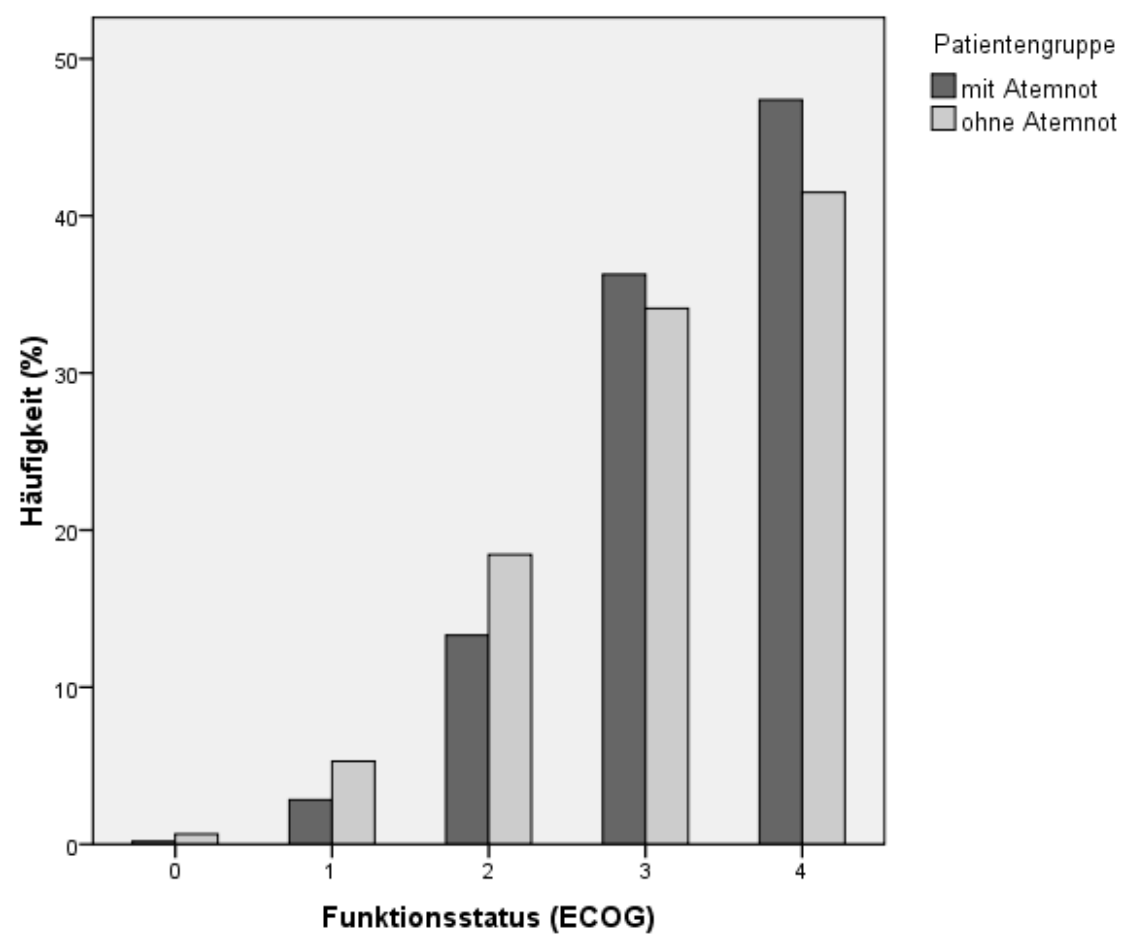

Abbildung 6: Funktionsstatus von Patienten mit und ohne Atemnot 
Dies zeigt sich auch im Vergleich der Atemnotprävalenzen in den Patientengruppen mit unterschiedlichem Funktionsstatus (Tabelle 30).

\begin{tabular}{|l|c|}
\hline & Prävalenz von Atemnot (\%) \\
\hline Funktionsstatus (ECOG) & \\
0 & 25,0 \\
1 & 38,4 \\
2 & 45,7 \\
3 & 55,3 \\
4 & 57,1 \\
Funktionsstatus-Gruppen & \\
(ECOG) & \\
$0-2$ & \\
3 oder 4 & 43,8 \\
& 56,3 \\
Fehldaten $(6,3 \%)$ & 53,3 \\
\hline
\end{tabular}

Tabelle 30: Atemnotprävalenzen in Abhängigkeit vom Funktionsstatus

\subsubsection{Patientenverfügung und Pflegestufe}

Patientenverfügungen lagen für $21,9 \%$ aller Patienten mit Atemnot und $19,4 \%$ aller Nichtatemnotpatienten vor. Der Unterschied war nicht signifikant.

Eine Pflegestufe existierte für 27,5\% aller Patienten mit Atemnot; $14,0 \%$ hatten eine Pflegestufe > I. $31,0 \%$ der Patienten ohne Atemnot hatten eine Pflegestufe; 17,6 \% besaßen eine Pflegestufe > I. Die Unterschiede waren mit $\mathrm{p}=0,002$ bzw. $\mathrm{p}<0,001$ signifikant (Tabelle 31).

\begin{tabular}{|l|l|l|l|}
\hline & Atemnot & Keine Atemnot & $\mathbf{p}$ \\
\hline Pflegestufe (\%) & & & \\
Keine & $1937(67,7)$ & $1562(63,5)$ & $<\mathbf{0 , 0 0 1}$ \\
I & $385(13,5)$ & $330(13,4)$ & \\
II & $318(11,1)$ & $316(12,8)$ & \\
III & $77(2,7)$ & $112(4,6)$ & \\
III+ & $5(0,2)$ & $6(0,2)$ & \\
Fehldaten & $138(4,8)$ & $134(5,5)$ & \\
Ja & $1937(67,7)$ & $1562(63,5)$ & $\mathbf{0 , 0 0 2}$ \\
Nein & $785(27,5)$ & $764(31,0)$ & \\
Keine/ I & $2322(81,2)$ & $1892(76,9)$ & $<\mathbf{0 , 0 0 1}$ \\
$>$ I & $400(14,0)$ & $434(17,6)$ & \\
\hline
\end{tabular}

Tabelle 31: Pflegestufen bei Patienten mit und ohne Atemnot 


\subsubsection{Weitere Symptome und Probleme}

Die meisten Patienten mit Atemnot zeigten neben Atemnot weitere Symptome. Besonders häufig fanden sich zusätzlich Schwäche (97,8 \%), Müdigkeit (93,0 \%), Appetitlosigkeit (87,4\%), Schmerz $(78,9 \%)$ und Anspannung (77,4 \%). Bei der Aufsummierung aller Symptome zur mittleren Symptomlast ergab sich ein Wert von 8,5 (Spannweite 0-12, SD 2,1). Entsprechend fand sich nach Addition aller Symptomintensitäten eine mittlere Intensitätslast von 17,1 (Spannweite 0-36, SD 5,7).

Patienten ohne Atemnot litten ebenfalls am häufigsten an Schwäche (96,3\%), Müdigkeit (90,4 \%), Appetitlosigkeit (82,2 \%), Schmerz (75,7\%) und Anspannung (69,0\%). Für die mittlere Symptomlast ergab sich ein Wert von 7,0 (SD 2,2), für die mittlere Intensitätslast 13,7 (SD 5,4).

In 11 der 12 Symptomkategorien hatten Patienten mit Atemnot größere Häufigkeiten und stärkere Intensitäten als Patienten ohne Atemnot. Entsprechend lagen die mittlere Symptomlast und die mittlere Intensitätslast von Patienten mit Atemnot signifikant über denen der Patienten ohne Atemnot. Das Symptom Desorientiertheit bildete eine Ausnahme. Für 33,4 \% aller Patienten mit Atemnot und für 34,3\% aller Patienten ohne Atemnot wurde „Desorientiertheit“ dokumentiert. Der Unterschied war nicht signifikant (Abbildung 7, Tabelle 32, Abbildung 8, Abbildung 9, Tabelle 53 im Anhang).

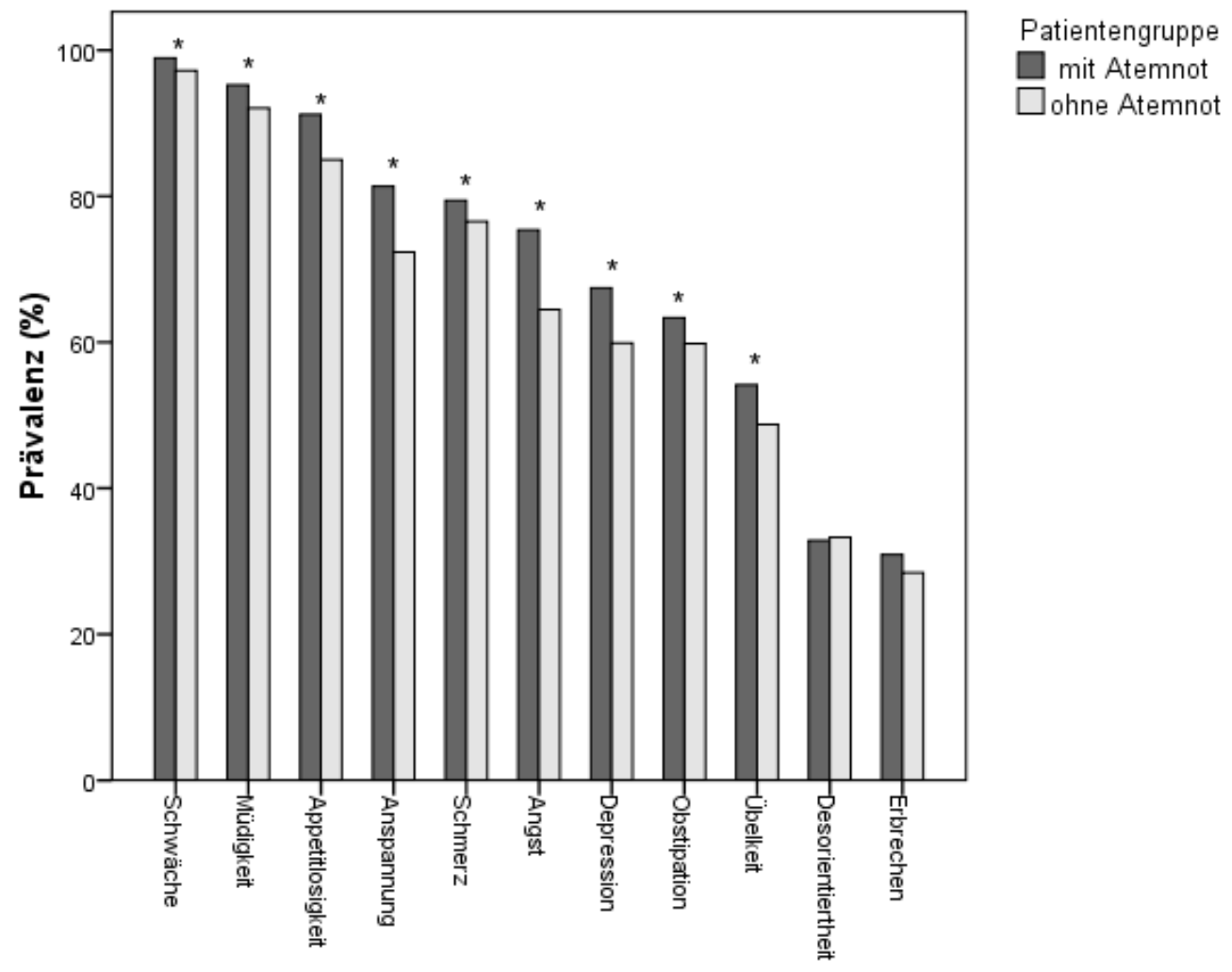

Abbildung 7: Symptomprävalenzen bei Patienten mit und ohne Atemnot (*: signifikanter Unterschied) 


\begin{tabular}{|l|l|l|l|}
\hline & Atemnot & Keine Atemnot & $\mathbf{p}$ \\
\hline Symptomlast & & & \\
(max. 12 Symptome) & & & \\
Mittelwert & 8,5 & 7,0 & $<\mathbf{0 , 0 0 1}$ \\
SD & 2,1 & 2,2 & \\
& & \\
Intensitätslast & & & \\
(max. 36 Punkte) & & 13,7 & \\
Mittelwert & 17,1 & 5,4 & $\mathbf{0 , 0 0 1}$ \\
SD & 5,7 & & \\
\hline
\end{tabular}

Tabelle 32: Symptom- und Intensitätslasten von Patienten mit und ohne Atemnot

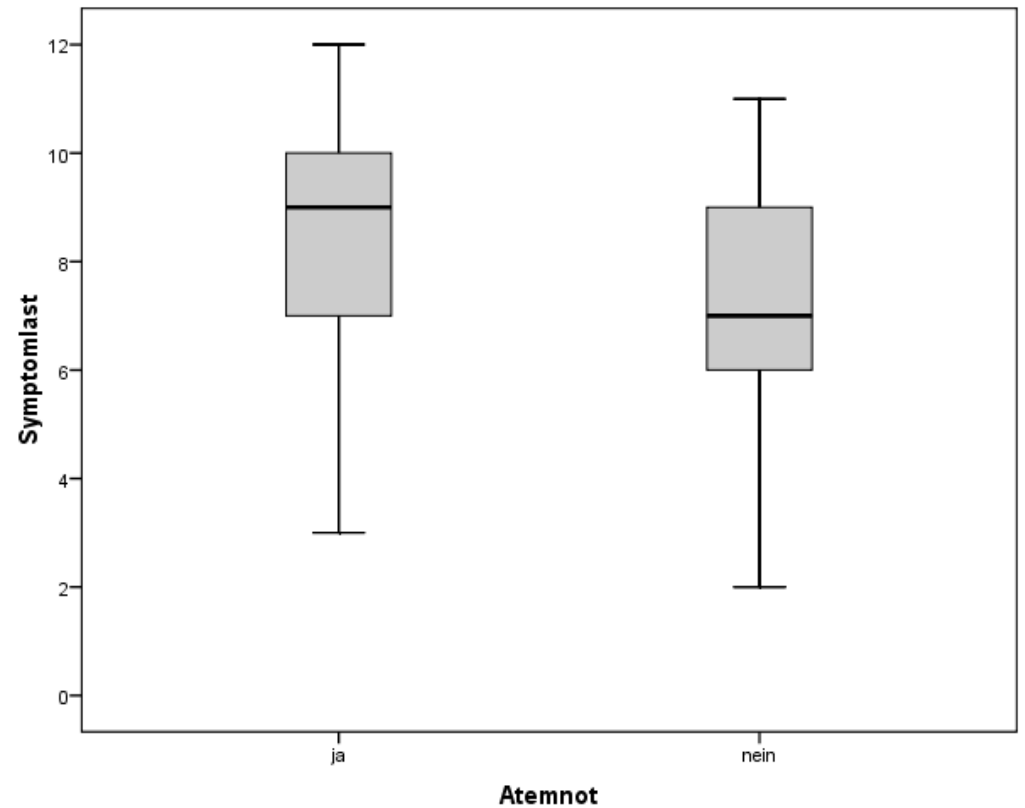

Abbildung 8: Vergleich der Symptomlast von Patienten mit und ohne Atemnot

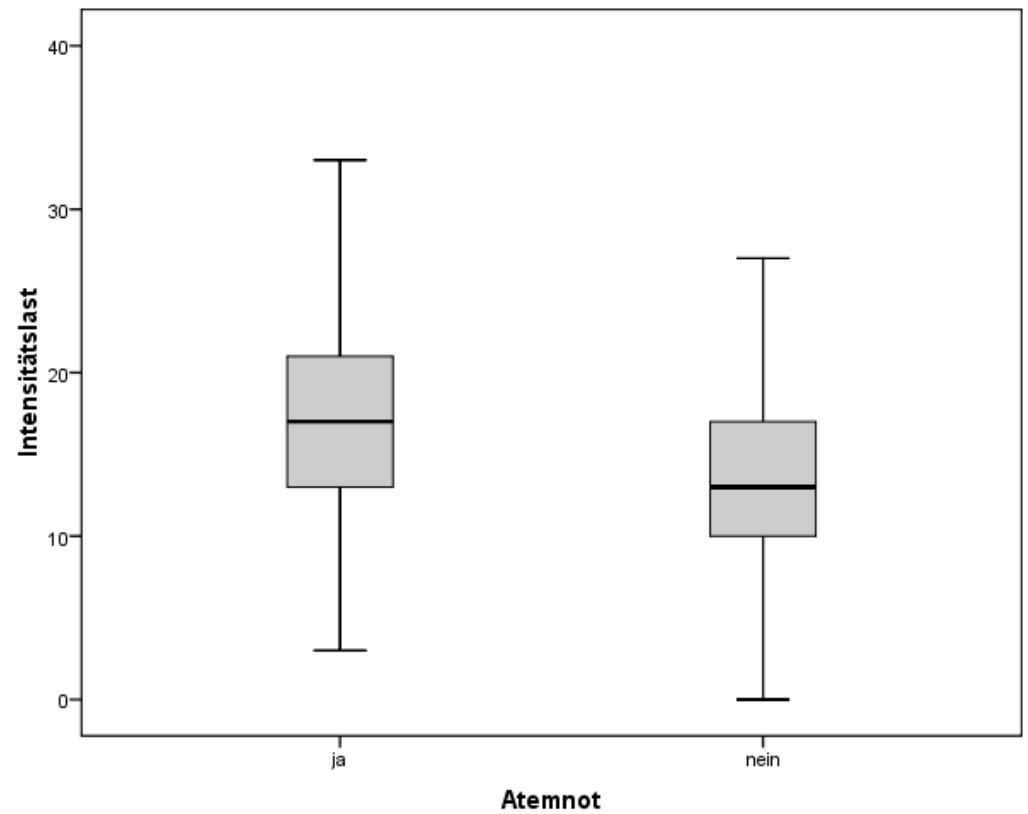

Abbildung 9: Vergleich der Intensitätslast von Patienten mit und ohne Atemnot 


\section{Weitere Probleme:}

Patienten mit Atemnot benötigten in 92,9 \% der Fälle Hilfe bei den Aktivitäten des täglichen Lebens, 74,0 \% zeigten eine Überforderung der Familie, 64,8 \% benötigten Unterstützung bei der Organisation der Versorgung und bei 37,0 \% war eine Wundversorgung notwendig. Der Vergleich mit den Patienten ohne Atemnot ist Abbildung 10 und Tabelle 54 im Anhang zu entnehmen. In allen vier Problemkategorien hatten Patienten mit Atemnot signifikant größere Häufigkeiten und signifikant höhere Intensitäten.

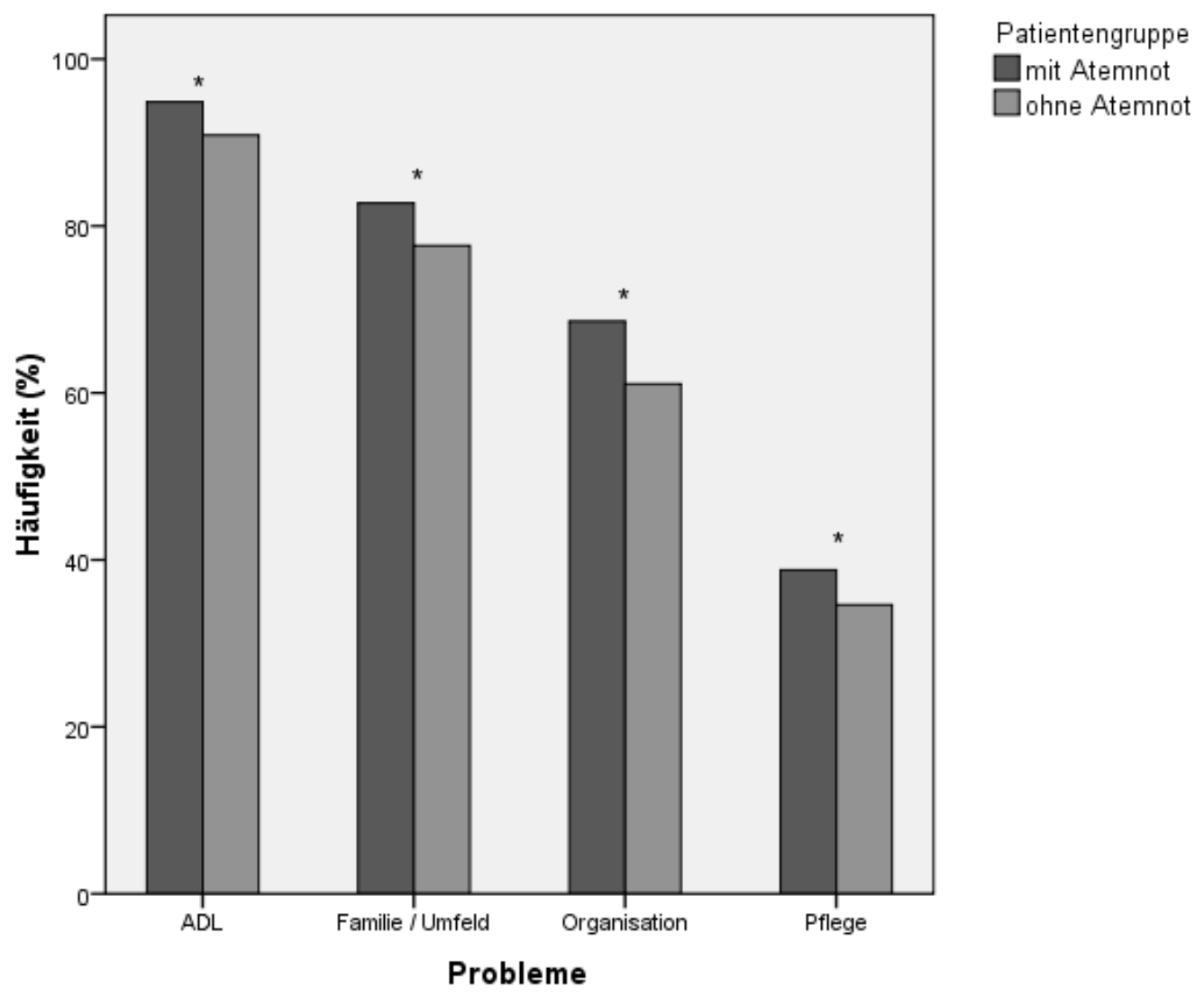

Abbildung 10: Häufigkeit von Problemen bei Patienten mit und ohne Atemnot (*: signifikanter Unterschied)

\subsubsection{Medikamente und Therapien}

\section{Medikamente:}

Als Medikation erhielten Patienten mit Atemnot bei Aufnahme in eine spezialisierte stationäre Palliativeinrichtung am häufigsten Opioide der WHO-Stufe III (62,9\%), gefolgt von Schmerzmitteln der WHO-Stufe I (51,2 \%) und Magenschutzpräparaten (46,3\%). Gleiches fand sich bei den Patienten ohne Atemnot mit nahezu identischen Häufigkeiten (Tabelle 55 im Anhang). 
Signifikant häufiger erfolgte bei Patienten mit Atemnot eine Verordnung von Antibiotika, Diuretika und Kardiaka/ Antihypertensiva. Auch Sedativa/ Anxiolytika wurden häufiger gegeben; der Unterschied war jedoch nicht signifikant.

Neuroleptika wurden dagegen signifikant seltener bei Patienten mit Atemnot verordnet (Abbildung 11).

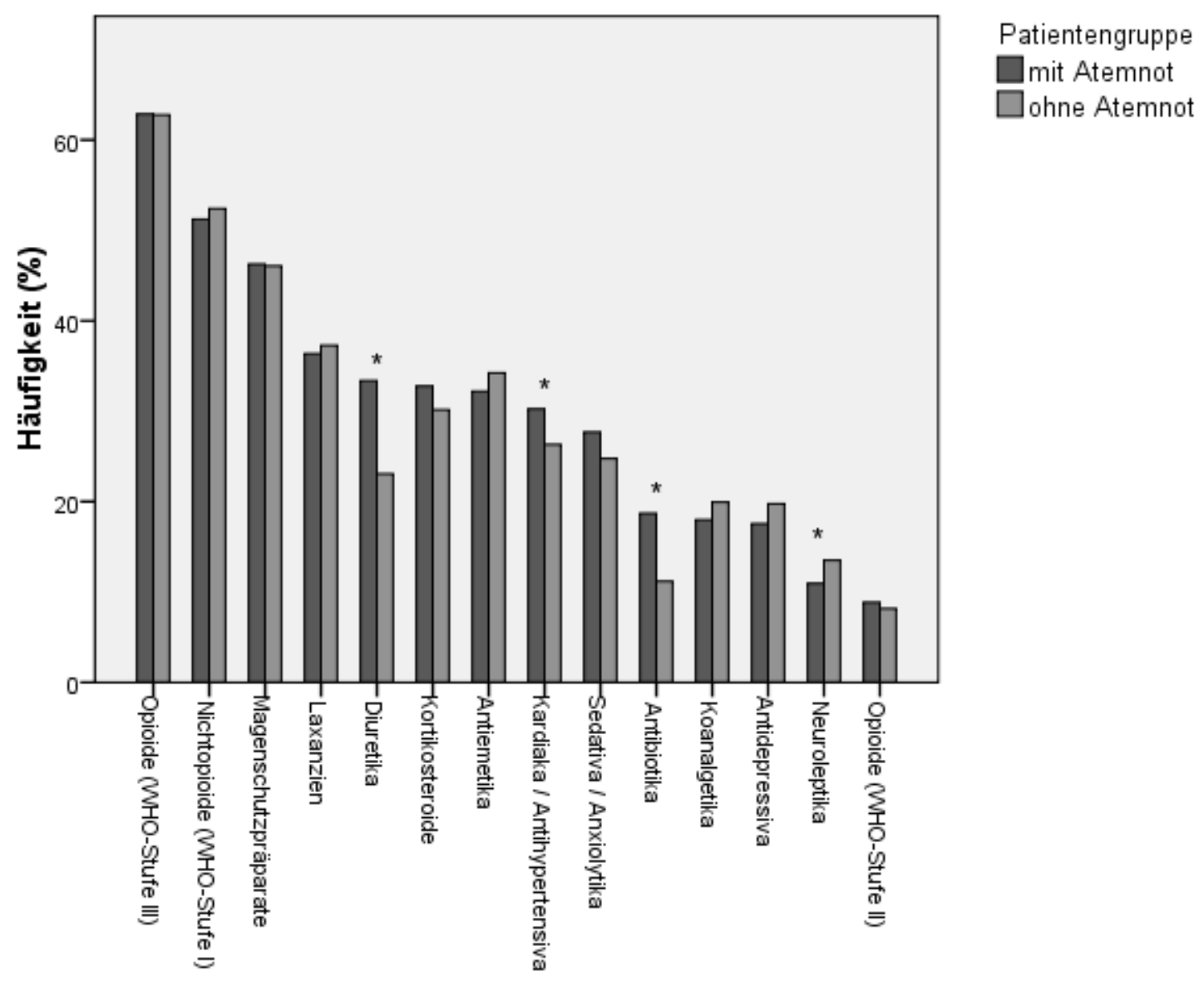

Abbildung 11: Häufigkeit der Verordnung von Medikamenten bei Patienten mit und ohne Atemnot bei Aufnahme in eine spezialisierte stationäre Palliativeinrichtung (*: signifikanter Unterschied)

\section{Maßnahmen und Therapien:}

Häufig durchgeführte Maßnahmen bzw. Therapien bei Patienten mit Atemnot waren Angehörigenbetreuung (45,1\%), intravenöse $(35,9 \%)$ und subkutane $(28,9 \%)$ Medikamentengabe und multimodale Schmerztherapie (20,2\%). Bei den Patienten ohne Atemnot ergab sich die gleiche Reihenfolge mit Angehörigenbetreuung (44,4\%), intravenöser (27,6\%) und subkutaner (26,7 \%) Medikamentengabe und multimodaler Schmerztherapie (20,5\%).

Signifikant häufiger wurden bei Patienten mit Atemnot eine intravenöse Medikamentengabe sowie Pleura- und Aszitespunktionen durchgeführt (Tabelle 56 im Anhang, Abbildung 12). 


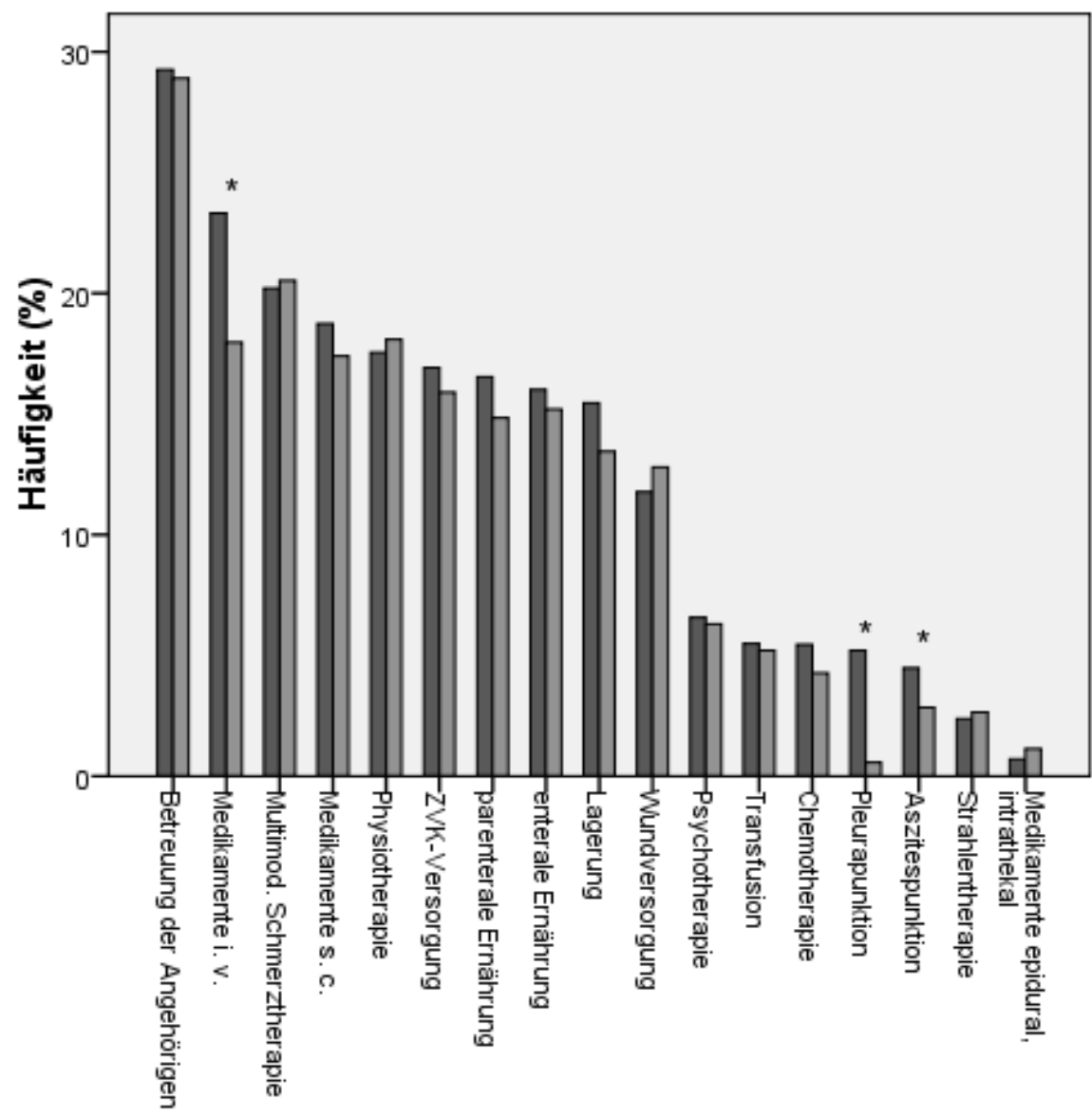

Patientengruppe

$\square$ mit Atemnot

$\square$ ohne Atemnot

Abbildung 12: Häufigkeit von Maßnahmen und Therapien bei Patienten mit und ohne Atemnot (*: signifikanter Unterschied)

\subsubsection{Liegedauer}

Die mittlere Liegedauer in der stationären Einrichtung lag für Patienten mit Atemnot bei 11,7 Tagen (SD: 10,9 Tage). Für Patienten ohne Atemnot fand sich eine mittlere Liegedauer von 13,6 Tagen (SD: 11,7). Der Unterschied war signifikant.

Unterteilt man die Patienten weiter in Verstorbene und Nicht-Verstorbene, wird deutlich, dass der Unterschied in der Liegedauer nur in der Gruppe der Verstorbenen besteht (9,9 Tage bei Patienten mit Atemnot vs. 12,8 Tage bei Patienten ohne Atemnot). Die Liegedauer der Nicht-Verstorbenen war in den Gruppen mit und ohne Atemnot annähernd gleich (14,2 Tage vs. 14,5 Tage) (Tabelle 33, Abbildung 13). 


\begin{tabular}{|c|c|c|c|}
\hline & Atemnot & Keine Atemnot & $\mathbf{p}$ \\
\hline $\begin{array}{l}\text { Liegedauer (d) } \\
\text { Mittelwert } \\
\text { Median } \\
\text { Spannweite } \\
\text { SD } \\
\text { Fehldaten (\%) }\end{array}$ & $\begin{array}{l}11,7 \\
9 \\
0-90 \\
10,9 \\
409(14,3 \%)\end{array}$ & $\begin{array}{l}13,6 \\
11 \\
0-87 \\
11,7 \\
423(17,2)\end{array}$ & $<0,001$ \\
\hline $\begin{array}{l}\text { Nicht-Verstorbene } \\
\text { (N=1966) } \\
\text { Mittelwert } \\
\text { Median } \\
\text { Spannweite } \\
\text { SD } \\
\text { Fehldaten (\%) }\end{array}$ & $\begin{array}{l}14,2 \\
12 \\
0-90 \\
10,9 \\
0\end{array}$ & $\begin{array}{l}14,5 \\
12 \\
0-87 \\
11,5 \\
0\end{array}$ & 0,55 \\
\hline $\begin{array}{l}\text { Verstorbene } \\
\text { (N=2042) } \\
\text { Mittelwert } \\
\text { Median } \\
\text { Spannweite } \\
\text { SD } \\
\text { Fehldaten (\%) }\end{array}$ & $\begin{array}{l}9,9 \\
7 \\
0-85 \\
10,7 \\
0\end{array}$ & $\begin{array}{l}12,8 \\
9 \\
0-81 \\
11,5 \\
1(0,1)\end{array}$ & $<0,001$ \\
\hline
\end{tabular}

Tabelle 33: Liegedauer von Patienten mit und ohne Atemnot, auch in Abhängigkeit vom Verlauf

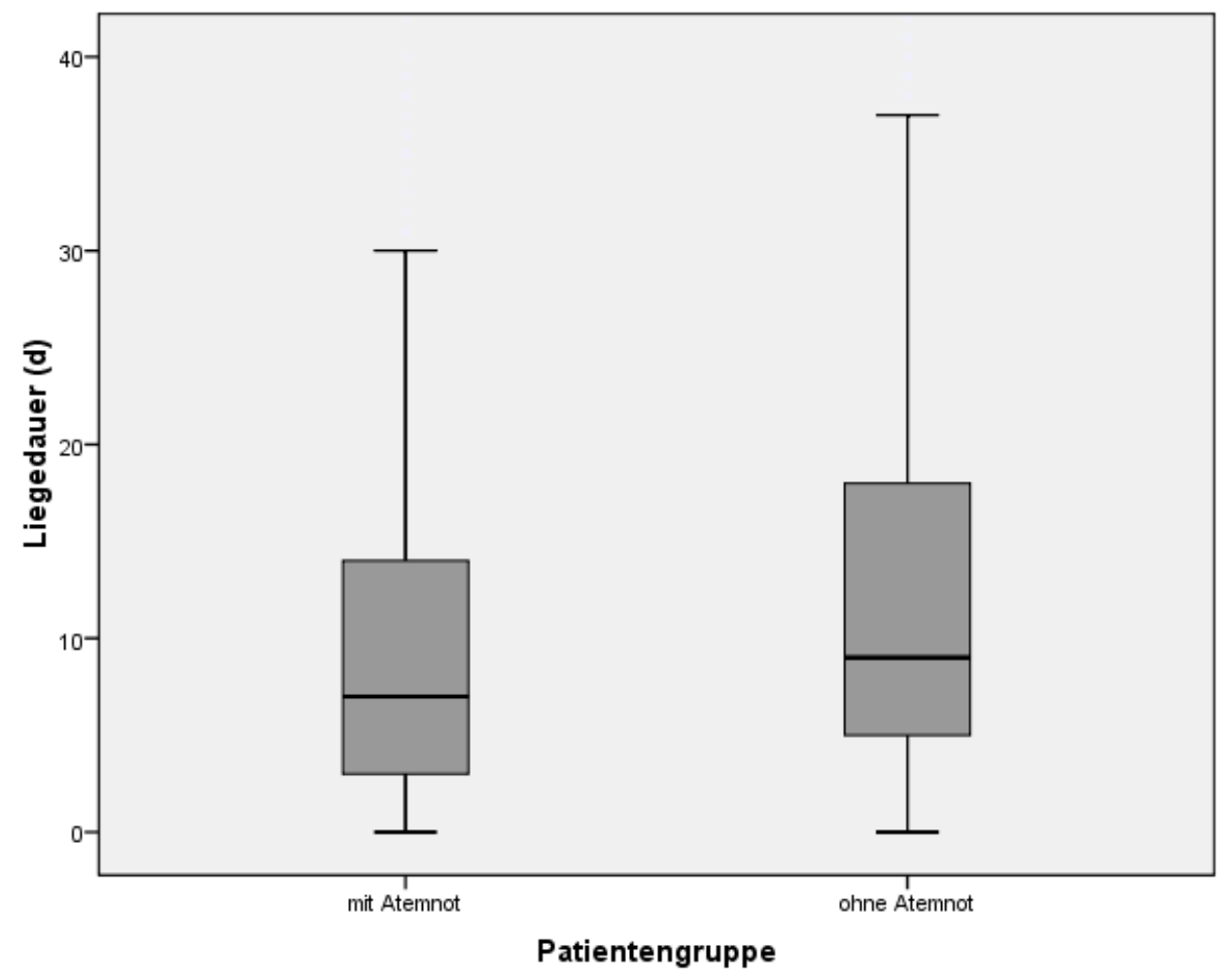

Abbildung 13: Liegedauer verstorbener Patienten mit und ohne Atemnot 
Bei der Betrachtung der Atemnotintensitäten zeigte sich eine signifikant abnehmende Liegedauer mit zunehmender Atemnotintensität. Nach weiterer Aufteilung der Patienten in Verstorbene und NichtVerstorbene ergab sich ein signifikanter Unterschied in der Liegedauer bei unterschiedlichen Atemnotintensitäten nur für die Gruppe der Verstorbenen. In der Gruppe der Nicht-Verstorbenen war die Liegedauer für Patienten mit unterschiedlichen Atemnotintensitäten annähernd gleich (Tabelle 34, Abbildung 14).

\begin{tabular}{|c|c|c|c|c|c|}
\hline & $\begin{array}{l}\text { Keine } \\
\text { Atemnot }\end{array}$ & Leicht & Mittel & Schwer & $\mathbf{p}$ \\
\hline Anzahl (\%) & $2460(46,2)$ & $1165(21,9)$ & $936(17,6)$ & $759(14,3)$ & \\
\hline $\begin{array}{l}\begin{array}{l}\text { Liegedauer } \\
\text { (d) }\end{array} \\
\text { Mittelwert } \\
\text { Median } \\
\text { Spannweite } \\
\text { SD } \\
\text { Fehldaten (\%) }\end{array}$ & $\begin{array}{l}13,6 \\
11 \\
0-87 \\
11,7 \\
423(17,2)\end{array}$ & $\begin{array}{l}12,7 \\
10 \\
0-88 \\
11,5 \\
153(13,1)\end{array}$ & $\begin{array}{l}11,6 \\
9 \\
0-90 \\
10,6 \\
146(15,6)\end{array}$ & $\begin{array}{l}10,0 \\
8 \\
0-81 \\
10,1 \\
110(14,5)\end{array}$ & $<0,001$ \\
\hline $\begin{array}{l}\text { Nicht- } \\
\text { Verstorbene } \\
\text { (N=1966) } \\
\text { Mittelwert } \\
\text { Median } \\
\text { Spannweite } \\
\text { SD } \\
\text { Fehldaten (\%) }\end{array}$ & $\begin{array}{l}14,5 \\
12 \\
0-87 \\
11,5 \\
0\end{array}$ & $\begin{array}{l}14,8 \\
13 \\
0-88 \\
11,9 \\
0\end{array}$ & $\begin{array}{l}13,8 \\
12 \\
0-90 \\
10,7 \\
0\end{array}$ & $\begin{array}{l}13,5 \\
12 \\
0-62 \\
8,8 \\
0\end{array}$ & 0,79 \\
\hline $\begin{array}{l}\text { Verstorbene } \\
\text { (N=2042) } \\
\text { Mittelwert } \\
\text { Median } \\
\text { Spannweite } \\
\text { SD } \\
\text { Fehldaten (\%) }\end{array}$ & $\begin{array}{l}12,8 \\
9 \\
0-81 \\
11,5 \\
1(0,1)\end{array}$ & $\begin{array}{l}11,3 \\
8 \\
0-85 \\
11,2 \\
0\end{array}$ & $\begin{array}{l}10,1 \\
7 \\
0-69 \\
10,4 \\
0\end{array}$ & $\begin{array}{l}8,1 \\
4 \\
0-81 \\
10,2 \\
0\end{array}$ & $<0,001$ \\
\hline
\end{tabular}

Tabelle 34: Liegedauer von Patienten mit unterschiedlichen Atemnotintensitäten, auch in Abhängigkeit vom Verlauf 


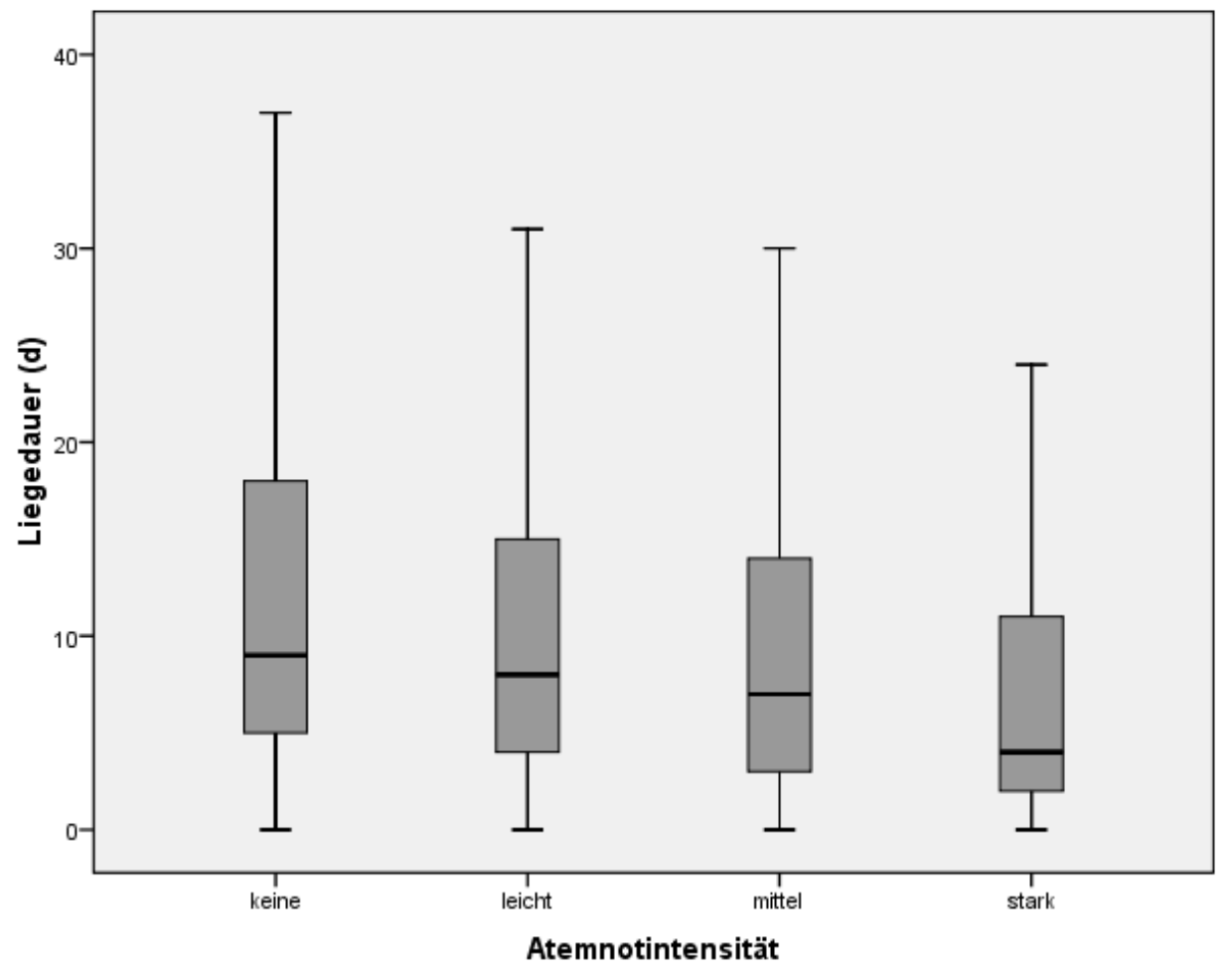

Abbildung 14: Liegedauer verstorbener Patienten mit unterschiedlichen Atemnotintensitäten

\subsubsection{Sterbefälle während des stationären Aufenthaltes}

43,5 \% aller Patienten mit Atemnot verstarben während des stationären Aufenthaltes, 33,8 \% wurden aus den Einrichtungen entlassen beziehungsweise lebten am Ende der Dokumentationsphase (22,7 \% Fehldaten). Bei den Patienten ohne Atemnot verstarben 32,4\%, 40,7 \% wurden entlassen beziehungsweise lebten am Ende der Dokumentationsphase (26,9 \% Fehldaten). Der Anteil Verstorbener war im Atemnotkollektiv signifikant höher als im Nicht-Atemnotkollektiv (Tabelle 35).

\begin{tabular}{|l|l|l|l|}
\hline & Atemnot & Keine Atemnot & p \\
\hline Verlauf (\%) & & & \\
Nicht-Verstorbene & $966(33,8)$ & $1000(40,7)$ & $<\mathbf{0 , 0 0 1}$ \\
Verstorbene & $1244(43,5)$ & $798(32,4)$ & \\
Fehldaten & $650(22,7)$ & $662(26,9)$ & \\
\hline
\end{tabular}

Tabelle 35: Verlauf bei Patienten mit und ohne Atemnot 
Beim Vergleich der Gruppen mit unterschiedlichen Atemnotintensitäten ergab sich ein signifikant zunehmender Anteil Verstorbener mit steigender Atemnotintensität (Tabelle 36, Abbildung 15).

\begin{tabular}{|l|c|c|c|c|c|}
\hline & \multicolumn{1}{|c|}{$\begin{array}{c}\text { Keine } \\
\text { Atemnot }\end{array}$} & \multicolumn{1}{|c|}{ Leicht } & Mittel & Schwer & p \\
\hline Anzahl (\%) & $2460(46,2)$ & $1165(21,9)$ & $936(17,6)$ & $759(14,3)$ & \\
\hline $\begin{array}{l}\text { Verlauf (\%) } \\
\text { Nicht- }\end{array}$ & $1000(40,7)$ & $439(37,7)$ & $311(33,2)$ & $216(28,5)$ & $<\mathbf{0 , 0 0 1}$ \\
$\begin{array}{l}\text { Verstorbene } \\
\text { Verstorbene } \\
\text { Fehldaten }\end{array}$ & $798(32,4)$ & $467(40,1)$ & $406(43,4)$ & $371(48,9)$ & \\
\hline
\end{tabular}

Tabelle 36: Verlauf bei Patienten mit unterschiedlichen Atemnotintensitäten

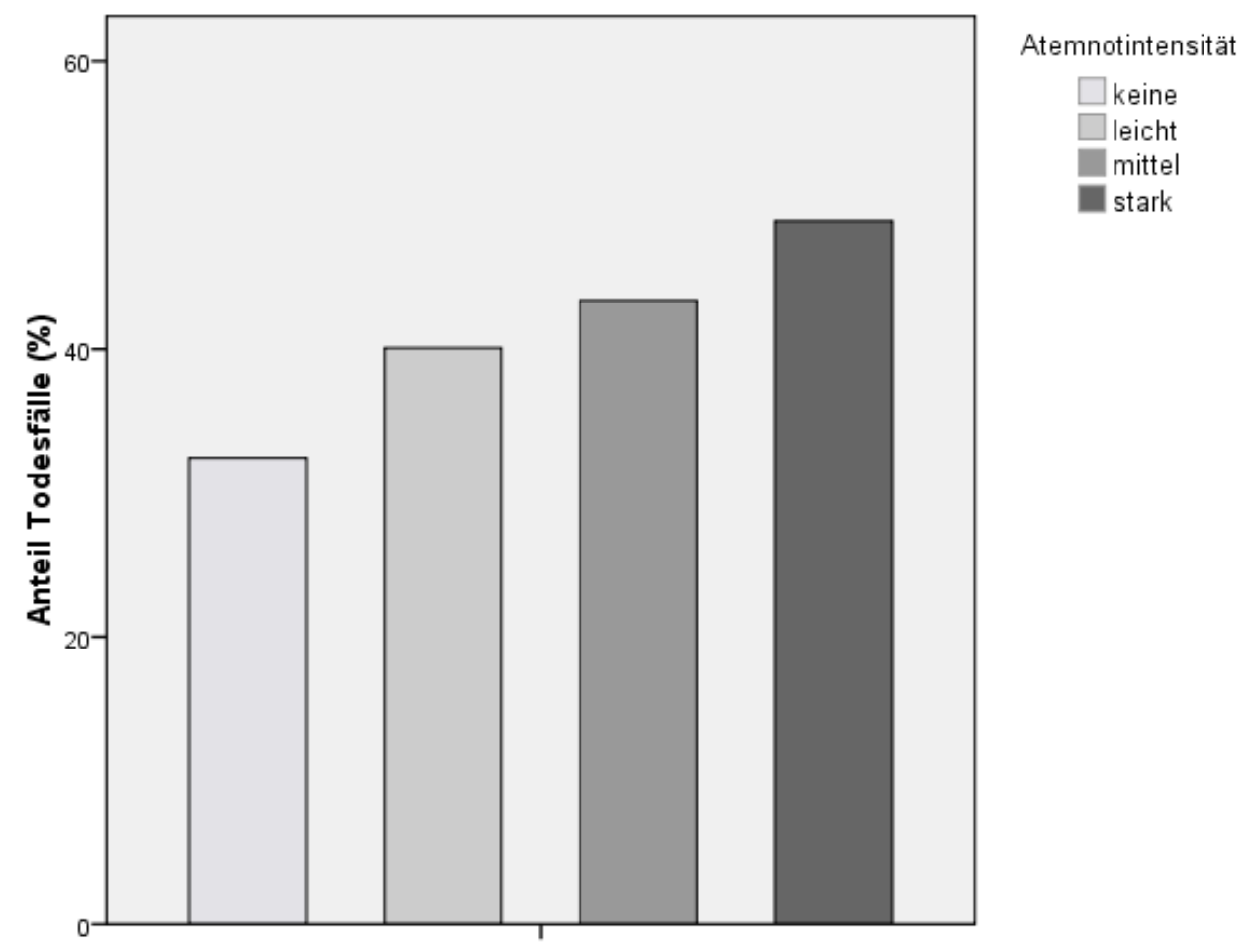

Abbildung 15: Anteil der Todesfälle in den Patientengruppen mit unterschiedlichen Atemnotintensitäten 
Die Unterschiede in der Überlebenszeit zeigten sich auch im Kaplan-Meier-Modell. Die mittlere Überlebenszeit für Patienten mit Atemnot betrug 22,5 Tage, für Patienten ohne Atemnot 28,5 Tage. Der Unterschied war mit einem p log Rank <0,001 signifikant (Abbildung 16).

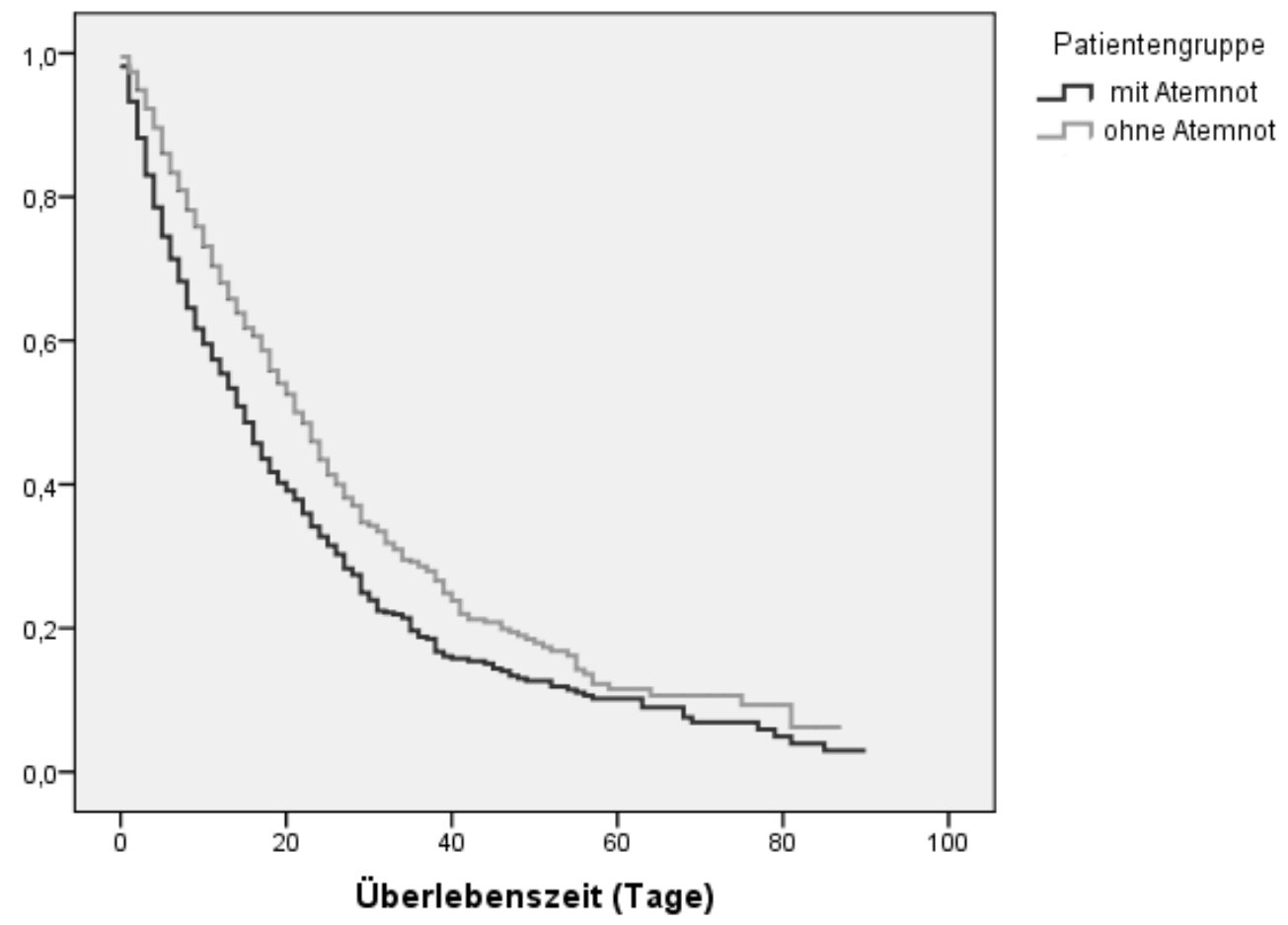

Abbildung 16: Kaplan-Meier-Modell für die Überlebenszeit von Patienten mit und ohne Atemnot

Entsprechendes zeigte sich im Vergleich der unterschiedlichen Atemnotintensitäten. Patienten mit leichter Atemnot hatten eine mittlere Überlebenszeit von 26,1 Tagen, Patienten mit mittlerer Atemnot eine Überlebenszeit von 21,5 Tagen und Patienten mit starker Atemnot eine von 17,8 Tagen. Auch dieser Unterschied war mit p log Rank <0,001 signifikant (Abbildung 17). 


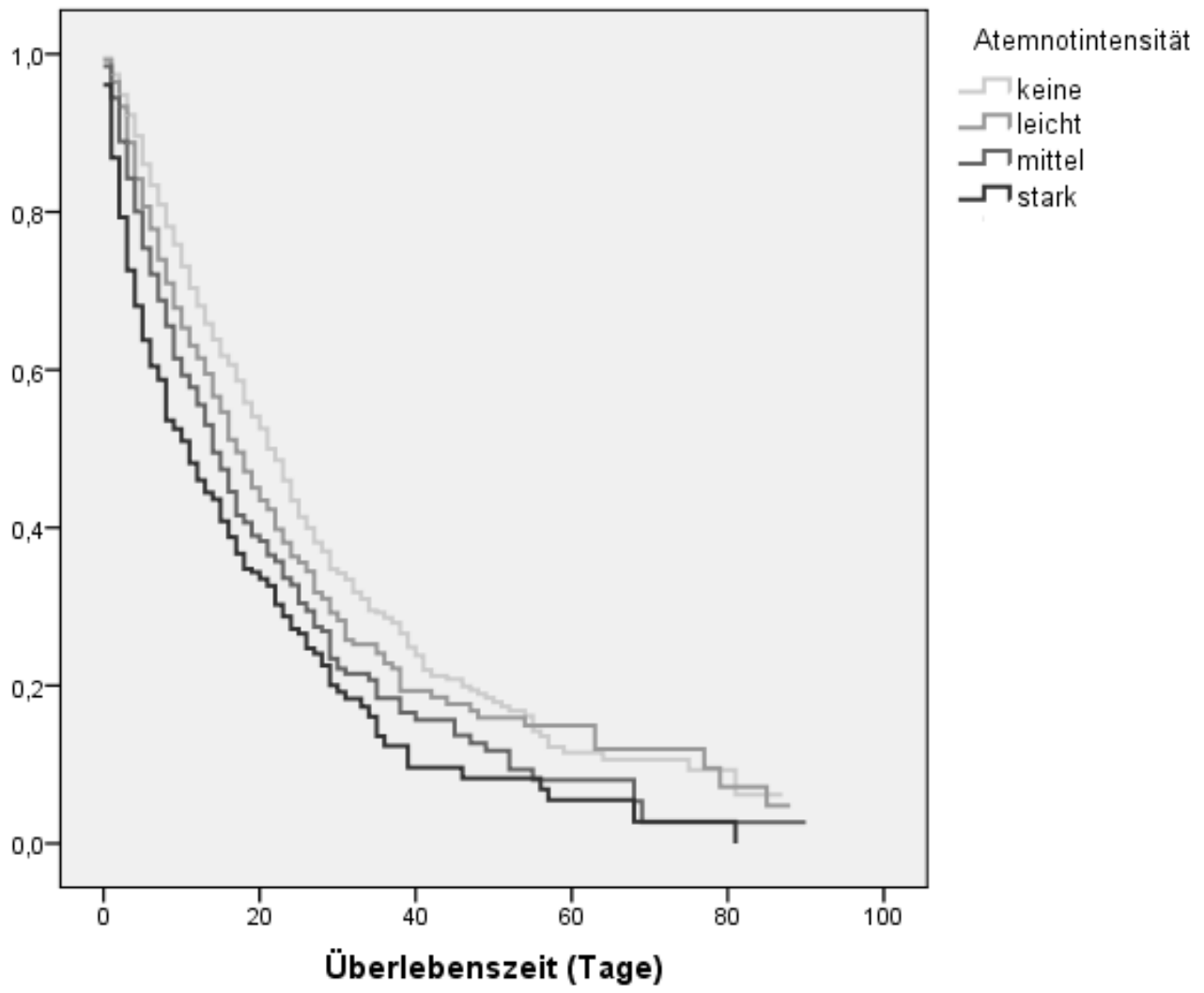

Abbildung 17: Kaplan-Meier-Modell für die Überlebenszeit von Patienten mit unterschiedlichen Atemnotintensitäten

Die Atemnotprävalenz bei den verstorbenen Patienten betrug 60,9\%, bei den Nicht-Verstorbenen lag sie bei $49,1 \%$. Der Unterschied war signifikant.

Es fanden sich im Verstorbenenkollektiv signifikant häufiger Patienten mit stärkerer Atemnot als bei den Nicht-Verstorbenen. (Tabelle 37).

\begin{tabular}{|l|l|l|l|}
\hline & Nicht-Verstorbene & Verstorbene & $\mathbf{p}$ \\
\hline Anzahl & 1966 & 2042 & \\
\hline $\begin{array}{l}\text { Atemnot (\%) } \\
\text { Ja }\end{array}$ & $966(49,1)$ & $1244(60,9)$ & $<\mathbf{0 , 0 0 1}$ \\
Nein & $1000(50,9)$ & $798(39,1)$ & \\
\hline Atemnotintensität & & & \\
(\%) & & $798(39,1)$ & $<\mathbf{0 0 0 1}$ \\
Keine & $1000(50,9)$ & $467(22,9)$ & \\
Leicht & $439(22,3)$ & $406(19,9)$ & \\
Mittel & $311(15,8)$ & $371(18,2)$ & \\
Schwer & $216(11,0)$ & & \\
\hline
\end{tabular}

Tabelle 37: Vorkommen und Intensität von Atemnot in Abhängigkeit vom Verlauf 
Für Patienten mit Atemnot zeigte sich mit einer Odds Ratio (OR) von 1,61 ein signifikant erhöhtes Risiko zu versterben. Dabei stieg die Wahrscheinlichkeit mit zunehmender Atemnotintensität (Tabelle $38)$.

\begin{tabular}{|c|c|c|c|c|}
\hline Variable & $\mathbf{N}$ & OR & CI 95 \% & p \\
\hline $\begin{array}{c}\text { Atemnot } \\
\text { Ja }\end{array}$ & 2210 & 1,61 & $1,42-1,83$ & $<\mathbf{0 , 0 0 1}$ \\
Nein & 1798 & 1 & & \\
& & & & \\
Atemnotintensität & & 1 & & \\
0 & 1798 & 1,33 & $1,14-1,57$ & $<\mathbf{0 , 0 0 1}$ \\
1 & 906 & 1,64 & $1,37-1,95$ & $<\mathbf{0 , 0 0 1}$ \\
2 & 717 & 2,15 & $1,78-2,61$ & $<\mathbf{0 , 0 0 1}$ \\
3 & 587 & & & \\
\hline
\end{tabular}

Tabelle 38: Risiko zu versterben in Abhängigkeit vom Vorhandensein bzw. der Intensität von Atemnot 


\subsection{Assoziierte Faktoren mit Atemnot}

\subsubsection{Korrelation ausgewählter Merkmale mit Atemnot}

Die Stärke und Richtung der Korrelation zwischen dem Vorhandensein bzw. der Intensität von Atemnot und ausgewählten Merkmalen sind Tabelle 57 im Anhang zu entnehmen.

Ein schwacher, aber signifikant positiver Zusammenhang konnte für das männliche Geschlecht, einen schlechteren Funktionsstatus, eine maligne Neubildung (Karzinom oder Metastasen) der Lunge, das Lungenkarzinom, Metastasen, Lungen-, Pleura- und Lymphknotenmetastasen, COPD, Schmerz, Übelkeit, Obstipation, Schwäche, Appetitlosigkeit, Müdigkeit, Depression, Angst, Anspannung und die Intensitätslast gezeigt werden.

Ein mittelstarker Zusammenhang mit Atemnot ergab sich für die Symptomlast.

Ein schwacher signifikant negativer Zusammenhang fand sich für das kolorektale Karzinom, das Pankreaskarzinom, das Prostatakarzinom, das Magenkarzinom sowie für die Liegedauer und das Überleben.

Bei der Analyse der Korrelationen von ausgewählten Merkmalen mit der Atemnotintensität ergab sich ein sehr ähnliches Bild bzgl. Richtung, Stärke und Signifikanz des Zusammenhangs. Unterschiede zeigten sich bei der Peritonealkarzinose. Hier fand sich ein schwach signifikant negativer Zusammenhang mit der Atemnotintensität. Ein schwacher signifikant positiver Zusammenhang ergab sich für die Herzinsuffizienz und die Symptomlast. Mittelstark positiv war der Zusammenhang mit der Intensitätslast.

Kein Zusammenhang ließ sich hingegen bei Schmerz und Übelkeit finden.

\subsubsection{Einzelfaktoranalyse}

Bei der Ermittlung des Risikos (prediction), beim Vorhandensein einzelner Faktoren auch unter dem Symptom Atemnot zu leiden, wurde jeweils das Odds Ratio (OR) berechnet. Die Ergebnisse sind Tabelle 39 und Tabelle 40 zu entnehmen.

Kein signifikantes Risiko ergab sich für sämtliche Altersgruppen, Wohnsituation: allein Lebende, ECOG 1 und 2 sowie für leichte Schwäche.

Signifikant erhöht war das Risiko für das männliche Geschlecht (OR 1,2), für Patienten mit einer nicht-malignen Grunderkrankung (OR 1,3), für ein malignes Tumorgeschehen in der Lunge (OR 2,71), für die ECOG Stufen 3 (OR 3,7) und 4 (OR 4,0) sowie für Patienten mit vorhandener Schwäche (OR 2,7), Depression (OR 1,4) und Angst (OR 1,7). Insbesondere zeigten sich in den Symptomkategorien für höhere Symptomintensitäten größere Risiken, an Atemnot zu leiden. 


\begin{tabular}{|c|c|c|c|c|}
\hline Variable & $\mathbf{N}$ & OR & CI $95 \%$ & $\mathbf{p}$ \\
\hline $\begin{array}{c}\text { Geschlecht } \\
\text { weiblich } \\
\text { männlich } \\
\end{array}$ & $\begin{array}{l}2839 \\
2420 \\
\end{array}$ & $\begin{array}{c}1 \\
1,20 \\
\end{array}$ & $1,07-1,33$ & 0,001 \\
\hline $\begin{array}{c}\text { Altersgruppen } \\
<65 \\
65-74 \\
75-84 \\
>84 \\
\end{array}$ & $\begin{array}{c}1910 \\
1679 \\
1233 \\
382 \\
\end{array}$ & $\begin{array}{c}1,22 \\
1,21 \\
1,10 \\
1 \\
\end{array}$ & $\begin{array}{l}0,98-1,52 \\
0,97-1,51 \\
0,88-1,39\end{array}$ & $\begin{array}{l}0,07 \\
0,09 \\
0,40\end{array}$ \\
\hline $\begin{array}{c}\text { Allein lebend } \\
\mathrm{Ja} \\
\mathrm{Nein} \\
\end{array}$ & $\begin{array}{c}814 \\
2428 \\
\end{array}$ & $\begin{array}{c}1 \\
1,08 \\
\end{array}$ & $0,92-1,27$ & 0,34 \\
\hline $\begin{array}{c}\text { Krebs } \\
\text { Ja } \\
\text { Nein } \\
\end{array}$ & $\begin{array}{c}5014 \\
289 \\
\end{array}$ & $\begin{array}{c}1 \\
1,30 \\
\end{array}$ & $1,02-1,66$ & $\mathbf{0 , 0 3}$ \\
\hline $\begin{array}{c}\text { Bösartige } \\
\text { Neubildung in } \\
\text { der Lunge } \\
\text { Ja } \\
\text { Nein } \\
\end{array}$ & $\begin{array}{l}2169 \\
3151 \\
\end{array}$ & $\begin{array}{c}2,71 \\
1 \\
\end{array}$ & $2,42-3,04$ & $<0,001$ \\
\hline $\begin{array}{c}\text { ECOG } \\
0 \\
1 \\
2 \\
3 \\
4\end{array}$ & $\begin{array}{c}20 \\
198 \\
782 \\
1759 \\
2227\end{array}$ & $\begin{array}{c}1 \\
1,87 \\
2,52 \\
3,71 \\
3,99\end{array}$ & $\begin{array}{c}0,65-5,35 \\
0,91-7,00 \\
1,34-10,26 \\
1,45-11,01\end{array}$ & $\begin{array}{c}0,24 \\
0,08 \\
\mathbf{0 , 0 1 1} \\
\mathbf{0 , 0 0 8}\end{array}$ \\
\hline
\end{tabular}

Tabelle 39: Einzelfaktoranalyse ausgewählter demographischer und klinischer Faktoren bzgl. des Risikos, an Atemnot zu leiden

\begin{tabular}{|c|c|c|c|c|}
\hline Variable & $\mathbf{N}$ & OR & CI $95 \%$ & $\mathbf{p}$ \\
\hline $\begin{array}{c}\text { Schwäche } \\
\text { Ja } \\
\text { Nein } \\
\\
0 \\
1 \\
2 \\
3 \\
\end{array}$ & $\begin{array}{c}5166 \\
95 \\
\\
95 \\
496 \\
1694 \\
2976\end{array}$ & $\begin{array}{c}2,69 \\
1 \\
1 \\
1,31 \\
2,17 \\
3,44 \\
\end{array}$ & $\begin{array}{l}0,82-2,10 \\
1,39-3,39 \\
2,21-5,35\end{array}$ & $\begin{array}{c}0,27 \\
\mathbf{0 , 0 0 1} \\
<\mathbf{0 , 0 0 1} \\
\end{array}$ \\
\hline $\begin{array}{c}\text { Depression } \\
\text { Ja } \\
\text { Nein } \\
\\
0 \\
1 \\
2 \\
3 \\
\end{array}$ & $\begin{array}{c}3187 \\
1784 \\
\\
1784 \\
1537 \\
1080 \\
570 \\
\end{array}$ & $\begin{array}{c}1,39 \\
1 \\
1 \\
1,33 \\
1,32 \\
1,72 \\
\end{array}$ & $\begin{array}{l}1,16-1,53 \\
1,14-1,54 \\
1,42-2,09 \\
\end{array}$ & $\begin{array}{l}<0,001 \\
<0,001 \\
<0,001\end{array}$ \\
\hline $\begin{array}{c}\text { Angst } \\
\text { Ja } \\
\text { Nein } \\
\\
0 \\
1 \\
2 \\
3 \\
\end{array}$ & $\begin{array}{c}3554 \\
1475 \\
\\
1475 \\
1643 \\
1238 \\
673\end{array}$ & $\begin{array}{c}1,69 \\
1 \\
1 \\
1,30 \\
1,94 \\
2,56 \\
\end{array}$ & $\begin{array}{c}1,123-1,50 \\
1,67-2,26 \\
2,11-3,10\end{array}$ & $\begin{array}{l}<0,001 \\
<0,001 \\
<0,001\end{array}$ \\
\hline
\end{tabular}

Tabelle 40: Einzelfaktoranalyse ausgewählter Symptome bzgl. des Risikos, an Atemnot zu leiden 


\subsubsection{Multiregressionsanalyse}

Als ein Ergebnis der Einzelfaktoranalyse wurde für die Kategorien „,malignes Tumorwachstum in der Lunge“, „Funktionsstatus“, „Schwäche“ und „Angst“ eine Multiregressionsanalyse durchgeführt (Tabelle 41). Im Vergleich zur Einzelfaktoranalyse zeigte sich in diesem Modell mit kombinierten Einflussfaktoren ein etwas höheres Risiko für das Vorhandensein von Atemnot bei malignem Tumorwachstum in der Lunge. Geringer fiel das Risiko für Funktionsstatus, Schwäche und Angst aus.

\begin{tabular}{|c|c|c|c|c|}
\hline Variable & $\mathbf{N}$ & OR & CI 95 \% & $\mathbf{p}$ \\
\hline $\begin{array}{c}\text { Malignom der } \\
\text { Lunge } \\
\text { Ja }\end{array}$ & 1944 & 2,72 & $2,40-3,07$ & $<\mathbf{0 , 0 0 1}$ \\
Nein & 2518 & 1 & & \\
\hline ECOG & & 1 & $0,50-4,42$ & 0,48 \\
0 & 20 & 1,48 & $0,69-5,77$ & 0,21 \\
1 & 190 & 1,99 & $0,97-8,11$ & 0,06 \\
2 & 768 & 2,81 & $1,11-9,27$ & $\mathbf{0 , 0 3}$ \\
3 & 1699 & 3,22 & $1,19-3,22$ & $\mathbf{0 , 0 0 8}$ \\
4 & 2007 & 1,96 & & \\
\hline $\begin{array}{c}\text { Schwäche } \\
\text { Ja }\end{array}$ & 4599 & 1 & $1,39-1,81$ & $<\mathbf{0 , 0 0 1}$ \\
Nein & 85 & 1,58 & & \\
\hline Angst & 3316 & 1 & & \\
Ja & 1368 & Nein &
\end{tabular}

Tabelle 41: Multiregressionsanalyse mit ausgewählten Faktoren bzgl. des Risikos, an Atemnot zu leiden

Bei der Bewertung der Güte der Multiregressionsanalyse ergaben sich die folgenden Ergebnisse (Tabelle 42):

\begin{tabular}{|l|c|c|c|}
\hline \multicolumn{1}{|c|}{ Parameter } & Test & Wert & p \\
\hline $\begin{array}{l}\text { Model performance } \\
\text { (Güte) }\end{array}$ & $\begin{array}{c}\text { Omnibus-Test der } \\
\text { Modellkoeffizienten }\end{array}$ & Chi-Quadrat 286,4 & $<\mathbf{0 0 1}$ \\
\hline $\begin{array}{l}\text { Goodness of fit } \\
\text { (Passung, Ist das Modell } \\
\text { lohnenswert?) }\end{array}$ & $\begin{array}{c}\text { Hosmer-Lemeshow- } \\
\text { Test }\end{array}$ & Chi-Quadrat 6,3 & 0,39 \\
\hline $\begin{array}{l}\text { Usefulness of the model } \\
\text { (Nützlichkeit, Wie viel } \\
\text { der Varianz wird durch } \\
\text { das Modell erklärt? }\end{array}$ & $\begin{array}{c}\text { Cox-\&-Snell- / } \\
\text { Nagelkerke-Test }\end{array}$ & $5,9-7,9 \%$ & \\
\hline
\end{tabular}

Tabelle 42: Tests zur Bewertung der Multiregressionsanalyse

Es zeigte sich zwar eine hohe Güte und eine gute Passung des Modells, eine Nützlichkeit war allerdings nicht gegeben: Durch das Modell konnten nur 5,9 bis 7,9\% der Varianz von Atemnot erklärt werden. 


\subsection{Fremd- und Eigeneinschätzung}

\subsubsection{Charakteristika von Patienten mit auswertbarem MIDOS-Bogen}

Von den 5.320 Patienten die im Rahmen der vorliegenden Studie ausgewertet wurden, lag für 2.655 (49,9 \%) zusätzlich ein auswertbarer MIDOS-Bogen mit einer Selbsteinschätzung von Symptomen vor. Nur diese Patientengruppe ging in die folgende Analyse ein. Sie wurde als „MIDOS-Gruppe“ bezeichnet.

Das mittlere Alter der Patienten der MIDOS-Gruppe betrug 66,9 Jahre (SD: 12,8 Jahre), 54,4 \% waren weiblich. Während des stationären Aufenthaltes lagen 87,4 \% der Patienten auf Palliativstationen, 12,6 \% lagen in einem stationären Hospiz. 95,8 \% litten an einer malignen Grunderkrankung, 15,4 \% hatten ein Lungenkarzinom. 71,9\% hatten einen niedrigen Funktionsstatus (ECOG 3 oder 4). 34,0 \% der Patienten verstarben im Verlauf des stationären Aufenthaltes, 47,9 \% konnten entlassen werden bzw. lebten am Ende der Dokumentationsphase (18,1\% Fehldaten).

Im Vergleich zu den Patienten ohne MIDOS-Bogen zeigten sich keine Unterschiede bei Alter, Geschlecht und im Auftreten eines Bronchialkarzinoms.

Patienten der MIDOS-Gruppe lagen jedoch signifikant häufiger auf einer Palliativstation $(87,4 \%$ vs. 71,6 \%, p<0,001), hatten einen signifikant besseren Funktionsstatus (ECOG 3/4 71,9 \% vs. 78,0 \%, $\mathrm{p}<0,001)$, waren signifikant häufiger an einem Malignom erkrankt $(95,8 \%$ vs. 92,7 \%, p<0,001), hatten eine signifikant längere mittlere Liegedauer (13,2 Tage vs. 11,8 Tage, p<0,001) und ein signifikant geringeres Risiko während des stationären Aufenthaltes zu versterben (34,0 \% vs. 42,7 \%, $\mathrm{p}<0,001)$ (Tabelle 43). 


\begin{tabular}{|c|c|c|c|c|}
\hline & GG & $\begin{array}{l}\text { MIDOS- } \\
\text { Gruppe }\end{array}$ & Ohne MIDOS & $\mathbf{p}$ \\
\hline Anzahl (\%) & $5320(100)$ & $2655(49,9)$ & $2665(50,1)$ & \\
\hline $\begin{array}{rr}\text { Anzahl (\%) } 2006 \\
2007 \\
2008\end{array}$ & $\begin{array}{l}1861(35,0) \\
2066(38,8) \\
1393(26,2)\end{array}$ & $\begin{array}{l}1025(38,6) \\
1019(38,4) \\
611(23,0)\end{array}$ & $\begin{array}{l}836(31,4) \\
1047(39,3) \\
782(29,3)\end{array}$ & \\
\hline $\begin{array}{l}\text { Alter } \\
\text { Mittelwert } \\
\text { Spannweite } \\
\text { SD } \\
\text { Fehldaten }(\%)\end{array}$ & $\begin{array}{l}67,2 \\
6-104 \\
13,0 \\
116(2,2)\end{array}$ & $\begin{array}{l}66,9 \\
14-100 \\
12,8 \\
57(2,1)\end{array}$ & $\begin{array}{l}67,6 \\
6-104 \\
13,1 \\
59(2,2)\end{array}$ & 0,06 \\
\hline $\begin{array}{l}\text { Geschlecht (\%) } \\
\text { weiblich } \\
\text { männlich } \\
\text { Fehldaten }\end{array}$ & $\begin{array}{l}2839(53,4) \\
2420(45,5) \\
61(1,1)\end{array}$ & $\begin{array}{l}1443(54,4) \\
1192(44,9) \\
20(0,8)\end{array}$ & $\begin{array}{l}1396(52,4) \\
1228(46,1) \\
41(1,5)\end{array}$ & 0,26 \\
\hline $\begin{array}{l}\text { Institution (\%) } \\
\text { PS } \\
\text { SH }\end{array}$ & $\begin{array}{l}4228(79,5) \\
1092(20,5)\end{array}$ & $\begin{array}{l}2320(87,4) \\
335(12,6)\end{array}$ & $\begin{array}{l}1908(71,6) \\
757(28,4)\end{array}$ & $<0,001$ \\
\hline $\begin{array}{l}\text { ECOG }(\%) \\
0 \\
1 \\
2 \\
3 \\
4 \\
\text { Fehldaten }\end{array}$ & $\begin{array}{l}20(0,4) \\
198(3,7) \\
782(14,7) \\
1759(33,1) \\
2227(41,9) \\
334(6,3)\end{array}$ & $\begin{array}{l}13(0,5) \\
117(4,4) \\
484(18,2) \\
919(34,6) \\
989(37,3) \\
133(5,0)\end{array}$ & $\begin{array}{l}7(0,3) \\
81(3,0) \\
298(11,2) \\
770(28,9) \\
1308(49,1) \\
201(7,5)\end{array}$ & $<0,001$ \\
\hline Median & 3 & 3 & 4 & \\
\hline $\begin{array}{l}\text { FS-Gruppen } \\
0-2 \\
3 / 4 \\
\text { Fehldaten }\end{array}$ & $\begin{array}{l}1000(18,8) \\
3986(74,9) \\
334(6,3))\end{array}$ & $\begin{array}{l}614(23,1) \\
1908(71,9) \\
133(5,0) \\
\end{array}$ & $\begin{array}{l}386(14,5) \\
2078(78,0) \\
201(7,5) \\
\end{array}$ & $<0,001$ \\
\hline $\begin{array}{l}\text { Maligne } \\
\text { Grunderkrankung } \\
(\%) \\
\text { Ja } \\
\text { Nein } \\
\text { Fehldaten }\end{array}$ & $\begin{array}{l}5014(94,3) \\
289(5,4) \\
17(0,3)\end{array}$ & $\begin{array}{l}2544(95,8) \\
105(4,0) \\
6(0,2)\end{array}$ & $\begin{array}{l}2470(92,7) \\
184(6,9) \\
11(0,4)\end{array}$ & $<0,001$ \\
\hline $\begin{array}{l}\text { Bronchialkarzinom } \\
(\%) \\
\text { Ja } \\
\text { Nein } \\
\text { Fehldaten }\end{array}$ & $\begin{array}{l}864(16,2) \\
4439(83,4) \\
17(0,3)\end{array}$ & $\begin{array}{l}408(15,4) \\
2241(84,4) \\
6(0,2)\end{array}$ & $\begin{array}{l}456(17,1) \\
2198(82,5) \\
11(0,4)\end{array}$ & 0,08 \\
\hline $\begin{array}{l}\text { Desorientiertheit } \\
\mathrm{Ja} \\
\text { Nein } \\
\text { Fehldaten }\end{array}$ & $\begin{array}{l}1802(33,9) \\
3303(62,1) \\
215(4,0)\end{array}$ & $\begin{array}{l}694(26,1) \\
1913(72,1) \\
48(1,8)\end{array}$ & $\begin{array}{l}1108(41,6) \\
1390(52,2) \\
167(6,3)\end{array}$ & $<0,001$ \\
\hline $\begin{array}{l}\text { Liegedauer (d) } \\
\text { Mittelwert } \\
\text { Median } \\
\text { Spannweite } \\
\text { SD } \\
\text { Fehldaten (\%) }\end{array}$ & $\begin{array}{l}12,5 \\
10 \\
0-90 \\
11,3 \\
832(15,6)\end{array}$ & $\begin{array}{l}13,2 \\
11 \\
0-90 \\
11,0 \\
321(12,1)\end{array}$ & $\begin{array}{l}11,8 \\
9 \\
0-88 \\
11,6 \\
511(19,2)\end{array}$ & $<0,001$ \\
\hline $\begin{array}{l}\text { Verlauf }(\%) \\
\text { Nicht-Verstorbene } \\
\text { Verstorbene } \\
\text { Fehldaten }\end{array}$ & $\begin{array}{l}1966(37,0) \\
2042(38,4) \\
1312(24,7)\end{array}$ & $\begin{array}{l}1271(47,9) \\
904(34,0) \\
480(18,1)\end{array}$ & $\begin{array}{l}695(26,1) \\
1138(42,7) \\
832(31,2)\end{array}$ & $<0,001$ \\
\hline
\end{tabular}

Tabelle 43: Charakteristika von Patienten mit und ohne MIDOS-Bogen 
Die mittlere Symptomlast der Patienten der MIDOS-Gruppe lag bei 7,8 Punkten (SD 2,2) von insgesamt 12 möglichen in der Fremdeinschätzung, die mittlere Intensitätslast betrug 15,2 (SD 5,6) von 36. In der Symptomlast ergab sich kein wesentlicher Unterschied zur Patientengruppe ohne MIDOS-Bogen (7,8 (SD 2,4)). Die Intensitätslast war jedoch in der MIDOS-Gruppe signifikant niedriger (Patientengruppe ohne MIDOS: 15,8, SD 5,9, p<0,001) (Tabelle 44).

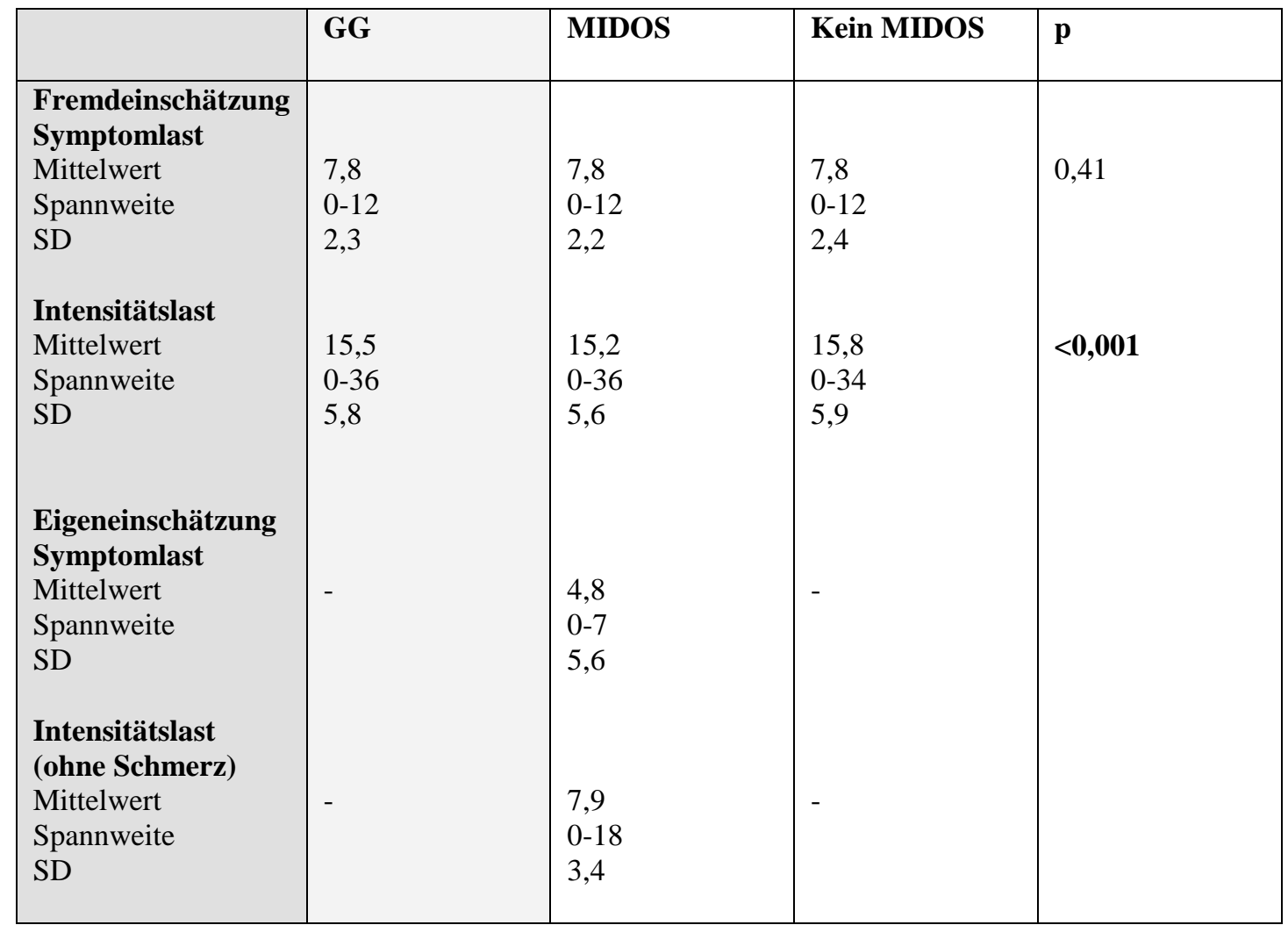

Tabelle 44: Symptom- und Intensitätslast in Fremd- bzw. Eigeneinschätzung bei Patienten mit und ohne MIDOS-Bogen

\subsubsection{Fremd- und Eigeneinschätzung von Atemnot}

Von den 2.655 Patienten der MIDOS-Gruppe äußerten sich 2.623 (98,8 \%) in der Selbsteinschätzung über das Symptom Atemnot (1,2\% Fehldaten).

Die Prävalenz von Atemnot betrug in der Fremdeinschätzung 53,4 \% im Vergleich zu 53,1 \% in der Eigeneinschätzung (Tabelle 45).

\begin{tabular}{|l|c|c|}
\hline Prävalenz von (\%) & $\begin{array}{c}\text { Fremdeinschätzung } \\
\text { (MIDOS) }\end{array}$ & Eigeneinschätzung \\
\hline Atemnot & 53,4 & 53,1 \\
\hline Leichter Atemnot & 23,2 & 22,8 \\
Mittlerer Atemnot & 16,7 & 17,5 \\
Schwerer Atemnot & 13,5 & 12,8 \\
\hline
\end{tabular}

Tabelle 45: Atemnotprävalenzen in Fremd- und Eigeneinschätzung 
80,9 \% der Patienten wurden bezüglich des Vorhandenseins beziehungsweise Fehlens von Atemnot durch das Fachpersonal richtig eingeschätzt (Sensitivität 81,8 \%, Spezifität 79,8 \%) (Tabelle 46).

\begin{tabular}{|l|l|c|c|c|}
\hline \multirow{2}{*}{\multicolumn{2}{|c|}{}} & \multicolumn{3}{|c|}{ Luftnot Eigeneinschätzung } \\
\cline { 2 - 5 } & Ja & Ja & Nein & Gesamt \\
\hline \begin{tabular}{l} 
Luftnot $\begin{array}{l}\text { Fremdeinschätzung } \\
\text { (MIDOS) }\end{array}$ \\
\cline { 2 - 4 }
\end{tabular} & Nein & 1153 & 968 & 1398 \\
\cline { 2 - 5 } & Gesamt & 1410 & 1213 & 1225 \\
\hline
\end{tabular}

Tabelle 46: Vergleich von Fremd- und Eigeneinschätzung für das Vorkommen von Atemnot

Bei der Beurteilung der Atemnotintensität wurden 34,3\% aller Patienten falsch eingeschätzt. 17,3\% der Patienten wurden in der Fremdeinschätzung überschätzt, 17,0 \% wurden unterschätzt (Tabelle 47).

\begin{tabular}{|l|l|c|c|c|c|c|}
\hline \multicolumn{2}{|c|}{} & \multicolumn{5}{|c|}{ Luftnotintensität Eigeneinschätzung } \\
\cline { 3 - 7 } & Keine & Leicht & Mittel & Schwer & Gesamt \\
\hline $\begin{array}{l}\text { Luftnotintensität } \\
\text { Fremdeinschätzung } \\
\text { (MIDOS) }\end{array}$ & Keine & 968 & 169 & 74 & 14 & 1225 \\
\cline { 2 - 7 } & Leicht & 188 & 305 & 86 & 30 & 609 \\
\cline { 2 - 7 } & Mittel & 42 & 98 & 227 & 72 & 439 \\
\cline { 2 - 7 } & Schwer & 15 & 34 & 77 & 224 & 350 \\
\cline { 2 - 7 } & Gesamt & 1213 & 606 & 464 & 340 & 2623 \\
\hline
\end{tabular}

Tabelle 47: Vergleich von Fremd- und Eigeneinschätzung für die Atemnotintensität

Die Übereinstimmung von Fremd- und Eigeneinschätzung konnte für das Vorhandensein von Atemnot (ja / nein) als „gut“ bewertet werden $(\kappa=0,62)$. Die Übereinstimmung in der Atemnotintensität ergab ein befriedigendes Ergebnis $(\kappa=0,50)($ Tabelle 48).

\begin{tabular}{|c|c|c|c|}
\hline \multirow{2}{*}{ Symptom } & \multirow{2}{*}{$\mathbf{N}$} & \multicolumn{2}{|c|}{$\begin{array}{c}\text { Grad der Übereinstimmung } \\
\text { (Cohen's } \mathbf{~})\end{array}$} \\
\cline { 3 - 4 } & & $\begin{array}{c}\text { Vorhandensein des } \\
\text { Symptoms }\end{array}$ & $\begin{array}{c}\text { Intensität des } \\
\text { Symptoms }\end{array}$ \\
\hline Atemnot & 2623 & 0,62 & 0,50 \\
\hline Schmerz & 2531 & 0,56 & n. a. \\
\hline Müdigkeit & 2606 & 0,37 & 0,33 \\
\hline Übelkeit & 2610 & 0,57 & 0,45 \\
\hline Verstopfung & 2557 & 0,56 & 0,46 \\
\hline Schwäche & 2601 & 0,26 & 0,36 \\
\hline Angst & 2521 & 0,38 & 0,32 \\
\hline
\end{tabular}

Tabelle 48: Grad der Übereinstimmung (Cohen's к) zwischen Selbst- und Fremdeinschätzung für das Vorhandensein und die Intensität der Symptome des MIDOS-Bogens 


\subsubsection{Fremd- und Eigeneinschätzung von weiteren Symptomen}

Auf dem MIDOS-Bogen wurden neben Atemnot die Symptome Schmerz, Müdigkeit, Übelkeit, Verstopfung, Schwäche, Angst erfasst. Der Grad der Übereinstimmung zwischen Selbst- und Fremdeinschätzung zum Vorhandensein des Symptoms sowie der Symptomintensität sind ebenfalls in Tabelle 48 aufgeführt.

Im Vergleich zu allen anderen Symptomen fanden sich sowohl für das Vorhandensein als auch für die Intensität des Symptoms bei Atemnot die größten Übereinstimmungen. Für Schmerz, Übelkeit und Verstopfung zeigten sich befriedigende Ergebnisse. Bei Müdigkeit, Schwäche und Angst gab es nur geringe Übereinstimmungen (,ausreichend“).

\subsubsection{Fremd- und Eigeneinschätzung von Atemnot bei Patienten mit unterschiedlichem Funktionsstatus}

Von den 2.655 Patienten der MIDOS-Gruppe lag für $2.495(98,9 \%)$ eine Einschätzung des Funktionsstatus (ECOG) vor. Nur diese Teilgruppe ging in die folgende Auswertung ein.

Der Grad der Übereinstimmung zwischen Selbst- und Fremdeinschätzung bzgl. des Symptoms Atemnot bei Patienten mit unterschiedlichem Funktionsstatus ist in Tabelle 49 und Abbildung 18 aufgeführt. Für alle ECOG-Stufen (bis auf ECOG 0) zeigte sich für die Prävalenz von Atemnot ein guter und für den Schweregrad von Atemnot ein befriedigender Grad der Übereinstimmung.

\begin{tabular}{|l|c|c|c|}
\hline \multirow{2}{*}{} & \multicolumn{2}{|c|}{$\begin{array}{c}\text { Grad der Übereinstimmung } \\
\text { (Cohen's к) }\end{array}$} & \multirow{2}{*}{ N (\%) } \\
\cline { 2 - 4 } & $\begin{array}{c}\text { Vorhandensein } \\
\text { von Atemnot }\end{array}$ & $\begin{array}{c}\text { Intensität } \\
\text { von Atemnot }\end{array}$ & \\
\hline Gesamt & 0,62 & 0,50 & $13(0,5)$ \\
\hline ECOG 0 & 0,16 & n. a. & $117(4,7)$ \\
\hline ECOG 1 & 0,71 & 0,65 & $482(19,3)$ \\
\hline ECOG 2 & 0,64 & 0,47 & $981(39,3)$ \\
\hline ECOG 3 & 0,61 & 0,49 & $902(36,2)$ \\
\hline ECOG 4 & 0,61 & 0,51 & \\
\hline
\end{tabular}

Tabelle 49: Grad der Übereinstimmung (Cohen's к) zwischen Selbst- und Fremdeinschätzung für das Vorhandensein und die Intensität von Atemnot in Abhängigkeit vom Funktionsstatus 


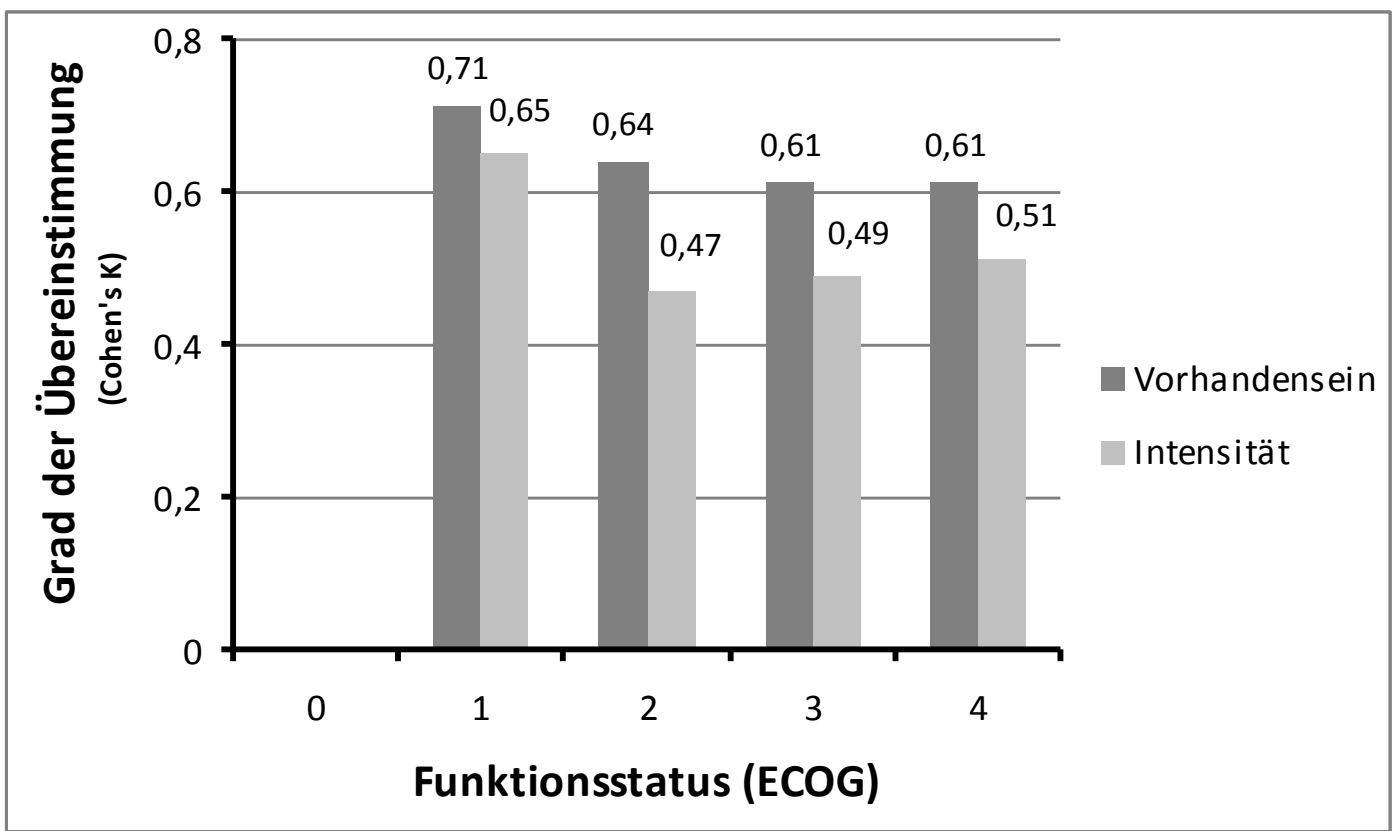

Abbildung 18: Grad der Übereinstimmung (Cohen's к) zwischen Selbst- und Fremdeinschätzung für das Vorhandensein und die Intensität von Atemnot in Abhängigkeit vom Funktionsstatus

\subsubsection{Fremd- und Eigeneinschätzung von Atemnot durch unterschiedliche Professionen}

Von den 2.655 Patienten mit MIDOS-Bogen konnten 2.091 (78,8 \%) bzgl. des Ausfüllens der Fremdeinschätzung einer einzelnen Profession zugeordnet werden. Nur diese Teilgruppe ging in die folgende Auswertung ein.

$79,6 \%$ dieser Patienten wurden durch einen ärztlichen Mitarbeiter beurteilt, 18,2 \% durch das Pflegepersonal. Weitere professionelle Betreuer wie Psychologen, Sozialarbeiter oder Seelsorger waren nur selten $(<2 \%)$ für die Fremdeinschätzung zuständig.

Der Grad der Übereinstimmung zwischen Selbst- und Fremdeinschätzung bzgl. des Symptoms Atemnot ist in Tabelle 50 aufgeführt. Sowohl für das Vorhandensein von Atemnot als auch für die Atemnotintensität ergaben sich keine wesentlichen Unterschiede im Grad der Übereinstimmung zwischen ärztlichen Mitarbeitern und dem Pflegepersonal. 


\begin{tabular}{|l|c|c|c|}
\hline \multirow{2}{*}{} & \multicolumn{2}{|c|}{$\begin{array}{c}\text { Grad der Übereinstimmung } \\
\text { (Cohen's к) }\end{array}$} & \multirow{2}{*}{ N (\%) } \\
\cline { 2 - 3 } & $\begin{array}{c}\text { Vorkommen } \\
\text { von Atemnot }\end{array}$ & $\begin{array}{c}\text { Intensität } \\
\text { von Atemnot }\end{array}$ & \multirow{2}{*}{$2091(100)$} \\
\hline Alle & 0,62 & 0,49 & $1665(79,6)$ \\
\hline Arzt & 0,62 & 0,48 & $380(18,2)$ \\
\hline Pflegepersonal & 0,61 & 0,49 & $9(0,4)$ \\
\hline Psychologe & 0,78 & 0,67 & $31(1,5)$ \\
\hline Sozialarbeiter & 0,80 & 0,61 & $6(0,3)$ \\
\hline Sonstige & 0,57 & n. a. & \\
\hline
\end{tabular}

Tabelle 50: Grad der Übereinstimmung zwischen Selbst- und Fremdeinschätzung für das Vorhandensein und die Intensität von Atemnot in Abhängigkeit von der Profession. 


\section{Diskussion}

\subsection{Kernaussagen}

Atemnot ist ein häufiges Symptom von schwer kranken Patienten. Diese Studie analysiert erstmalig deutschlandweit das Symptom Atemnot bei Palliativpatienten, die sich in der Behandlung und Betreuung einer spezialisierten stationären Palliativeinrichtung (Palliativstation oder stationäres Hospiz) befanden.

Die Ergebnisse der Analyse sind zahlreich; fünf Kernaussagen stehen dabei im Mittelpunkt:

1. Mehr als die Hälfte aller Palliativpatienten $(53,8 \%)$ litten bei Aufnahme in eine Einrichtung der spezialisierten Palliativversorgung in Deutschland unter Atemnot.

2. Palliativpatienten mit Atemnot zeigten sich als eine stark belastete und deutlich eingeschränkte Patientengruppe. Im Vergleich zu Patienten ohne Atemnot besaßen sie einen geringeren Funktionsstatus. Neben Atemnot beklagten sie eine signifikant größere Anzahl weiterer Symptome in einer signifikant stärkeren Symptomausprägung.

3. Patienten mit Atemnot hatten eine höhere Wahrscheinlichkeit, während des stationären Aufenthaltes zu versterben. Das Risiko stieg mit zunehmender Atemnotintensität. Auch die Zeit bis zum Versterben korrelierte mit der Atemnotintensität: Patienten mit schwerer Atemnot verstarben im Durchschnitt früher als Patienten mit leichter Atemnot.

4. Es ließen sich bestimmte Faktoren ausmachen, die mit einem höheren Risiko für das gleichzeitige Bestehen von Atemnot einhergingen. Ein konkretes Modell zur Erklärung der Varianz von Atemnot konnte jedoch nicht entwickelt werden.

5. Für die Prävalenz von Atemnot gab es eine gute Übereinstimmung zwischen der Selbsteinschätzung durch den Patienten und der Fremdeinschätzung durch einen professionellen Betreuer. Allerdings waren die Ergebnisse bei der Einschätzung der Atemnotintensität nur befriedigend. 


\subsection{Diskussion der Ergebnisse}

\subsubsection{Prävalenz von Atemnot}

\subsubsection{Prävalenz von Atemnot allgemein}

Atemnot ist ein häufiges Symptom von schwer kranken Patienten. In der durchgeführten Analyse litten mit 53,8 \% mehr als die Hälfte aller Patienten bei Aufnahme in eine Einrichtung der spezialisierten Palliativversorgung in Deutschland unter dem Symptom in einer durchschnittlich mittelstarken Ausprägung.

In der Literatur, die sich mit Symptomen schwer kranker Patienten beschäftigt, findet sich bei der Beschreibung der Prävalenz des Symptoms Atemnot eine große Variabilität. Ein systematischer Review von Linsenmeier (2008) beschreibt eine schwierige Vergleichbarkeit von unterschiedlichen Studien über Atemnotprävalenzen bei Patienten mit fortgeschrittenen Krebserkrankungen aufgrund von uneinheitlichen Messinstrumenten und -zeitpunkten, Umgebungsbedingungen, und fehlendem Konsens über die Definition von Atemnot. Insgesamt aber handelt es sich bei Atemnot, auch wenn verschiedene fortgeschrittene Grundkrankheiten betrachtet werden, um ein häufig vorkommendes Symptom (Solano et al. 2006). In einer großen Untersuchung $(\mathrm{N}=194.017)$ über Ursachen, warum Krebspatienten in den letzten sechs Monaten ihres Lebens eine Notfallambulanz aufsuchten, zählten Bronchialkarzinom, Pneumonie und Atemnot zu den häufigsten fünf (Barbera et al. 2010).

Die Ergebnisse der vorliegenden Arbeit stehen im Einklang mit den Daten vergleichbarer großer internationaler Studien aus der Palliativmedizin. In einer großen Analyse hatten bereits Reuben und Mor (1986) über 1.754 Krebspatienten eine Atemnotprävalenz von 49,3\% 5 bis 6 Wochen vor Eintritt des Todes beschrieben. Walsh et al. (2000) fanden in ihrer Arbeit über 1.000 Patienten mit fortgeschrittenen Krebserkrankungen eine Atemnotprävalenz von $50 \%$. Eine aktuelle Arbeit von Currow et al. (2010) über 5.862 Palliativpatienten in Australien ergab eine Atemnotprävalenz von $50 \% 3$ Monate vor Lebensende mit einem Anstieg auf $65 \%$ in der letzten Lebenswoche.

\subsubsection{Prävalenz von Atemnot in ausgewählten Subgruppen der Grundgesamtheit}

Bei der Betrachtung der Atemnotprävalenz in ausgewählten Subgruppen der Grundgesamtheit konnten folgende nennenswerte Unterschiede festgestellt werden.

\section{Atemnotprävalenz in Abhängigkeit vom Alter:}

In der Untersuchung zeigte sich tendenziell eine sinkende Atemnotprävalenz mit steigendem Alter. Bei den unter 65-Jährigen hatten 54,8 \% aller Patienten Atemnot, jedoch nur noch 49,7 \% der über 84Jährigen. Ursächlich hierfür könnte eine größere Immobilität der Älteren sein, bei der das Vorliegen von Atemnot nicht mehr so stark empfunden wird bzw. auffällt wie bei Jüngeren, die sich noch stärker 
belasten. Ältere Patienten könnten zudem zurückhaltender bei der Artikulation von Beschwerden sein, um nicht zur Last zu fallen. Eventuell adaptiert sich der ältere Mensch auch leichter an eine eingeschränkte Atmung. Im klinischen Alltag werden bei älteren Menschen z. B. geringere Sauerstoffpartialdrücke als noch physiologisch angesehen (Herold 2009). Möglich wäre auch, dass Atemnot von den zuständigen Betreuern mit zunehmendem Alter als nicht ungewöhnlich und damit nicht bemerkenswert angesehen wird.

Letztere Hypothese kann mit den vorliegenden Daten jedoch nicht belegt werden. Wird nämlich bei der Einschätzung von Atemnot die Selbsteinschätzung vom MIDOS-Bogen zugrunde gelegt, findet sich eine gleichgerichtete Tendenz mit abnehmender Atemnotprävalenz bei steigendem Alter $(<65$ Jahre: 55,1 \%, 65-74 Jahre: 52,0 \%, 75-84 Jahre: 51,5 \%, >84 Jahre: 49,7 \% (Ergebnisse im Ergebnisteil nicht genannt.)).

Ganz im Gegensatz zu der vorliegenden Arbeit fanden Currow et al. (2009 b) in ihrer Studie zur Prävalenz chronischer Atemnot eine zunehmende Atemnotprävalenz mit steigendem Alter. Allerdings liegen ihrer Analyse Daten aus der Allgemeinbevölkerung und nicht von schwerkranken Patienten zugrunde.

\section{Atemnotprävalenz in Abhängigkeit vom Geschlecht:}

Beim männlichen Geschlecht fand sich mit 56,2 \% eine signifikant höhere Atemnotprävalenz als bei Frauen mit 51,7 \%. Es liegt nahe, als Ursache zunächst die Tatsache heranzuführen, dass fast doppelt so viele Männer wie Frauen als Grunderkrankung ein Bronchialkarzinom aufwiesen. Tatsächlich zeigte sich bei reiner Betrachtung der Bronchialkarzinom-Gruppe eine höhere, wenn auch nicht signifikante $(\mathrm{p}=0,12)$ Atemnotprävalenz bei den Männern $(76,1 \%)$ als bei den Frauen $(71,3 \%)$. In der Nicht-Bronchialkarzinom-Gruppe waren die Atemnotprävalenzen bei den Geschlechtern annähernd gleich. (Männer: 48,9 \%, Frauen: 48,7 \%, p=0,92). Es ist möglich, dass dieses vermehrte Auftreten von Atemnot bei männlichen Patienten mit Bronchialkarzinom eine Folge weiterer Faktoren wie Nikotinkonsum oder bestimmter Begleiterkrankungen sein könnte, die jedoch in dieser Arbeit nicht erhoben wurden.

Auch hier fanden Currow et al. (2009 b) ein abweichendes Ergebnis in der Allgemeinbevölkerung mit einer signifikant höheren Atemnotprävalenz bei Frauen.

Atemnotprävalenz in Abhängigkeit von der Einrichtung:

Patienten, die auf einer Palliativstation lagen, hatten mit 55,1 \% eine signifikant höhere Atemnotprävalenz als Patienten im Hospiz (48,4\%).

Palliativstationen und Hospize unterscheiden sich in Aufgabenbereich und Zielsetzung, was zur Folge hat, dass jeweils Patienten mit anderen Bedürfnissen und Problemen aufgenommen und betreut werden. Auf einer Palliativstation werden als einem Akutkrankenhaus zugehörige Einheit eher Patienten mit plötzlicher Zustandsverschlechterung oder Symptomexazerbation im Rahmen einer 
Krisensituation aufgenommen. Im Vordergrund der Bemühungen steht die Symptomkontrolle, so dass Patienten nach Möglichkeit die Einrichtung in gebessertem Zustand wieder verlassen können.

Im Gegensatz dazu ist die Hauptaufgabe des Hospizes die Begleitung Sterbender. Patienten werden im weit fortgeschrittenen Stadium ihrer Erkrankung, zumeist aber in stabilem Zustand, hierher verlegt, weil eine mitunter auch längerfristige Betreuung bis zum Tod zuhause nicht gewährleistet ist.

Vor diesem Hintergrund scheint das Ergebnis der Analyse erklärbar. Atemnot wird bei Auftreten oder Verschlimmerung sowohl vom Patienten als auch von den Angehörigen meist als höchst akut und lebensbedrohlich empfunden (Gysels et al. 2007). Das macht eine unter Umständen auch notfallmäßige Aufnahme der Patienten mit Atemnot auf eine Palliativstation wahrscheinlicher. Patienten mit akuten Zuständen werden eher nicht im Hospiz betreut.

\section{Atemnotprävalenz in Abhängigkeit von Diagnosen:}

Nicht unerwartet ergaben sich bei der Betrachtung von Patientengruppen mit unterschiedlichen Diagnosen die höchsten Atemnotprävalenzen beim Bronchialkarzinom (74,3\%) sowie bei Vorliegen von Pleura- (75,2\%) oder Lungenmetastasen $(67,9 \%)$. Diese Ergebnisse sind vergleichbar mit den Daten internationaler Studien. So bezifferten Reuben und Mor (1986) die Atemnotprävalenz von Krebspatienten mit primärer oder sekundärer Lungenbeteiligung auf $75 \%$. Dudgeon et al. (2001) fanden in ihrer Analyse von 923 Krebspatienten mit 84 \% eine deutlich höhere Atemnotprävalenz bei Patienten mit Bronchialkarzinom als bei Patienten mit anderen Krebsdiagnosen. Einschränkend muss hier jedoch erwähnt werden, dass nur $4 \%(\mathrm{~N}=37)$ aller untersuchten Patienten an einem Bronchialkarzinom erkrankt waren, im Gegensatz zu 22,4\% (N=642) in der hier vorliegenden Arbeit. Eine Erwähnung verdienen Patienten mit einem kolorektalen Karzinom als Grunderkrankung. Obwohl das kolorektale Karzinom mit 9,5\% zu den häufigsten Krebserkrankungen bei Patienten mit Atemnot zählt, wurde es bei ihnen signifikant seltener als bei Patienten ohne Atemnot gefunden. Auch Reuben und Mor (1986) hatten in ihrer Studie weniger Patienten mit kolorektalem Karzinom in der Atemnotgruppe (13,7\%) als in der Nicht-Atemnotgruppe (21,3\%) gezählt.

Ein Erklärungsansatz, dass Patienten mit kolorektalem Karzinom seltener eine pulmonale oder pleurale Metastasierung aufweisen könnten, konnte anhand der vorliegenden Daten nicht erhärtet werden. Krebspatienten mit einem kolorektalen Karzinom zeigten in 39,5\% der Fälle pulmonale oder pleurale Metastasen. Dieser Wert liegt noch über dem Durchschnitt aller Malignompatienten (36,6 \%). Anders stellte sich das Ergebnis bei Patienten mit den Tumorentitäten Pankreas-, Magen- und Prostatakarzinom dar, die ebenfalls signifikant seltener in der Atemnotgruppe zu finden waren. Hier zeigte ich mit 20,5\%,20,0 \% und 14,9\% tatsächlich eine deutlich seltenere pulmonale oder pleurale Metastasierung als im Durchschnitt.

Bemerkenswerterweise ergaben die vorliegenden Daten sogar eine niedrigere Atemnotprävalenz für Patienten mit pulmonalen und pleuralen Metastasen, wenn ein Kolonkarzinom vorlag. Ob dieses Ergebnis bedeuten kann, dass das kolorektale Karzinom oder seine pulmonalen bzw. pleuralen 
Metastasen protektive Faktoren für das Auftreten von Atemnot darstellen oder pulmonale oder pleurale Metastasen eines kolorektalen Karzinoms weniger aggressiv Atemnot erzeugen, ist rein spekulativ und sollte Gegenstand zukünftiger Forschungsprojekte sein. Eine schlüssige Begründung oder tragfähige Hypothese kann somit hier nicht gegeben werden und konnte auch in der Literatur nicht gefunden werden.

Nur 5,4\% aller Patienten hatten keine Tumorerkrankung als Hauptdiagnose. Dieser Anteil von Patienten ohne Tumorerkrankung in Palliativeinrichtungen ist vergleichbar mit früheren Arbeiten (Potter et al. 2003). Die häufigsten Diagnosen in dieser Patientengruppe waren die Folgen eines apoplektischen Insultes, Anämie, chronische Herzinsuffizienz, Multisystematrophie und COPD. Patienten ohne Tumorerkrankung litten häufiger an Atemnot (59,9\%) als Patienten mit einer Krebsdiagnose $(53,4 \%)$. Unter den Nicht-Krebsdiagnosen zeigten sich besonders hohe Atemnotprävalenzen für die COPD (90,9\%) und die chronische Herzinsuffizienz (75,0\%).

Eine Erklärung für die höhere Atemnotprävalenz bei den Nicht-Tumorpatienten könnte sein, dass diese häufig unter internistischen und neurologischen Erkrankungen leiden, die zum einen mit einer hohen Prävalenz und Inzidenz von Atemnot einhergehen (chronische Herzinsuffizienz, Anämie, COPD, ALS) und zum anderen aufgrund einer schlechteren Einschätzbarkeit ihrer Prognose sehr weit fortgeschritten sind (Bausewein et al. 2010 b).

Eine aktuelle Arbeit von Ostgathe et al. (2011) bestätigt das Ergebnis der vorliegenden Arbeit. In einer Analyse der HOPE-Daten von 2002 bis 2005 war Atemnot signifikant häufiger bei Patienten ohne eine Tumorerkrankung (40\%) als bei Patienten mit einer Tumorerkrankung (29\%) zu finden.

Burt et al. (2010) kamen in ihrer Arbeit über Unterschiede zwischen den Erfahrungen von 1.266 Patienten mit und ohne Tumorerkrankung in den letzten Lebensmonaten zu einem ähnlichen Ergebnis. Von den Patienten, die im Verlauf an einer Nicht-Tumorerkrankung verstarben, litten $73,9 \%$ an Atemnot. Im Vergleich dazu hatten aber nur 64,7 \% aller Tumorpatienten Atemnot. Der Unterschied war signifikant. Ein Erklärungsansatz der Arbeit war, dass Patienten ohne Tumorerkrankung, begründet durch ihren begrenzteren Zugang zu palliativmedizinischen Diensten, eine schlechtere Symptomkontrolle erfahren könnten. Ein weiteres und bemerkenswertes Ergebnis der Arbeit war, dass Nicht-Tumorpatienten Atemnot als weniger stark einschränkend empfanden (89,5\%) als Tumorpatienten $(94,0 \%)$.

Auch Currow et al. (2010) beschrieben bei 5.862 Palliativpatienten mit und ohne Tumorerkrankung eine signifikant höhere Atemnotintensität bei Patienten mit Nicht-Krebserkrankungen in den letzten drei Lebensmonaten. Patienten ohne Tumorerkrankung erfuhren Atemnot über eine länger anhaltende Periode, die Intensität des Symptoms änderte sich bis zum Tod jedoch nicht wesentlich. Dagegen zeigten Krebspatienten initial eine niedrigere Atemnotintensität mit einem deutlichen Anstieg in den letzten 10 Lebenstagen. Die Studie unterstreicht, dass die Einschätzung der Belastung durch Atemnot 
und die Erwartung bzgl. der Dynamik des Symptoms immer im Zusammenhang mit der ursächlichen Diagnose gesehen werden muss.

Ähnliches fanden Bausewein et al. (2010 a) in einer Arbeit zu Verläufen von Atemnot bei Patienten mit fortgeschrittenen Krebserkrankungen bzw. COPD über 6 Monate. Sie beschrieben für COPDPatienten eine Zunahme der Atemnot von einem Punkt auf der Borg-Skala in 6 Monaten. Bei Patienten mit fortgeschrittenen Krebserkrankungen ergab sich eine Zunahme der Atemnot im letzten Lebensmonat und kurz vor dem Tod. Zusätzlich fanden sie für beide Patientengruppen bei individuellerer Betrachtung vier verschiedene Verlaufsformen: fluktuierende, zunehmende, stabile und abnehmende Atemnot im Verlauf.

Eine aktuelle Studie von Stiel et al. (2010 c) ergab einen signifikanten Anstieg der NichtTumorpatienten auf deutschen Palliativstationen von 4,9 \% im Jahre 2004 auf 7,3 \% 2009. Insbesondere kam es zu einem Anstieg von Patienten mit Herz-Kreislauf-, neurologischen und pulmonologischen Erkrankungen. Dieser Trend könnte ein Hinweis dafür sein, dass NichtTumorpatienten sowohl von der Palliativmedizin als auch von anderen medizinischen Disziplinen zunehmend als bedürftige Patientengruppe wahrgenommen werden, die eine ähnlich intensive, wenn auch anders gewichtete, palliativmedizinische Betreuung benötigen, wie die Gruppe der Krebspatienten. Gleichzeitig hieße dies aber auch, dass Konzepte für die Palliativversorgung überdacht werden müssen, um der anders gearteten Symptomlast, -intensität und -dynamik adäquat zu begegnen. Insbesondere bei Zunahme von kardiovaskulären und pulmonologischen Erkrankungen wird mit dem Symptom Atemnot häufiger zu rechnen sein, was die Entwicklung effektiver symptomspezifischer Strategien unumgänglich macht.

Bemerkenswerterweise konnte mit den vorliegenden Daten bei 47,2 \% aller Palliativpatienten mit Atemnot keine maligne primäre oder sekundäre Neubildung in der Lunge oder eine sonstige chronische die Lunge belastende Erkrankung gezeigt werden.

Ein Teil dieser Patienten könnte dennoch an einer okkulten Lungenerkrankung leiden (subklinische Thrombembolien oder Metastasierung, bislang nicht diagnostizierte pulmonal arterielle Hypertonie, Lungenfibrose als Folge einer Radio- bzw. Chemotherapie).

Auch kann Atemnot bei fortgeschrittenen Erkrankungen ihren Ursprung primär extrapulmonal nehmen. Booth et al. (2008) nennen in diesem Zusammenhang u. a. Anämie, Kachexie, eingeschränkte Mobilität und psychische Erkrankungen. Ein weiterer Erklärungsansatz beschreibt Atemnot bei Schwerstkranken als Folge einer allgemeinen Skelettmuskelschwäche. Diese führt zu einer reduzierten Kraftentwicklung und Belastbarkeit. Um eine gewohnte Muskelkraft aufzubauen, muss mehr Muskelkraft geleistet werden. Das Ergebnis ist Belastungs- und schließlich auch Ruhedyspnoe (American Thoracic Society 1999). Für die chronische Herzinsuffizienz konnte bei 
fortgeschrittenen Krankheitsstadien eine ausgeprägtere Skelettmuskelschwäche nachgewiesen werden (Sullivan et al. 1990).

Zudem stellt Atemnot bei schwer kranken Patienten ein multifaktorielles Syndrom dar, das Folge komplizierter pathophysiologischer Vorgänge ist (Dudgeon und Lertzman 1998). Abernethy und Wheeler führten 2008 den Begriff der „totalen Atemnot“ (total dyspnoea) in Anlehnung an den von Dame Cicely Saunders geprägten Begriff „totaler Schmerz“ (total pain) ein. Sie beschreiben Atemnot als vierdimensionales Symptom, das sich auf physische, psychische, soziale und spirituelle Bereiche auswirkt und von diesen beeinflusst wird, was in der Therapie genutzt werden kann.

Viele Zusammenhänge sind in diesem Kontext noch unklar. Ein noch besseres Verständnis der der Atemnot zugrunde liegenden pathophysiologischen Mechanismen scheint essentiell, um die Entwicklung effektiverer Therapien zu ermöglichen. Aktuelle Forschung auf der Basis neurophysiologischer und bildgebender Verfahren (PET, MRT etc.) könnten hier weitere Erkenntnis bringen (Leupoldt et al. 2009).

Dass Atemnot häufig auch ohne direkte Beteiligung der Lunge vorkommt, findet darüber hinaus in der klinischen Praxis noch zu wenig Beachtung. Extrapulmonale Ursachen sollten bei der differentialdiagnostischen Ursachenforschung von Atemnot in die Überlegungen einbezogen werden.

Insgesamt ergab die vorliegende Untersuchung zur Atemnotprävalenz bei stationären Palliativpatienten, dass es in der Grundgesamtheit Untergruppen gibt, die ein besonders hohes Risiko für das Bestehen von Atemnot haben. Patienten mit einer höheren Atemnotprävalenz sind häufig Männer, jüngere Patienten, Patienten mit primärer oder sekundärer bösartiger Neubildung in der Lunge sowie Patienten ohne Krebserkrankungen. Medizinisches Personal sollte besonders bei diesen Risikogruppen bei Anamnese, Untersuchung und Therapie sensibler auf das Symptom Atemnot achten, um den Patienten eine noch optimalere und umfassendere Symptomkontrolle zu ermöglichen.

\subsubsection{Symptome und Funktionsstatus}

Die Analyse der Symptome demonstriert, dass Patienten mit Atemnot an einer bedeutsamen Anzahl weiterer belastender Symptome leiden. Bei Patienten mit Atemnot ergab sich sowohl bei der Anzahl der Symptome als auch bei der Symptomintensität eine signifikant höhere mittlere Gesamtsumme, was auf eine höhere Belastung durch körperliche wie seelische Symptome sowie eine höhere Gesamtbelastung dieser Patientengruppe hindeutet.

Fast alle untersuchten Symptome traten häufiger in der Atemnotgruppe auf. Eine besonders deutliche Differenz ergab sich bei den psychischen Symptomen Angst (71,6 \% vs. 61,2 \%), Anspannung (77,4 $\%$ vs. $69,0 \%)$ und Depression (63,1\% vs. $56,2 \%)$.

In der Studie von Bausewein et al. (2010 b) fanden sich ebenfalls deutliche Hinweise auf eine besonders hohe Symptombelastung bei Patienten mit Atemnot. Ein signifikanter Zusammenhang 
zwischen Atemnot und den psychischen Symptomen Angst bzw. Anspannung wurde bereits in den Arbeiten von Dudgeon und Lertzman (1998) sowie Smith et al. (2001) beschrieben.

Eine Sonderstellung nimmt das Symptom Desorientiertheit ein. Es fand sich als einziges Symptom ungefähr gleich häufig im Atemnot- wie im Nicht-Atemnotkollektiv in einer ähnlich starken Ausprägung. Nach Prüfung möglicher Confounder konnte keine wirkliche Begründung für diese Abweichung im Vergleich zu allen anderen Symptomen gefunden werden. Möglicherweise handelt es sich um einen reinen Zufallsbefund. Auch in der Literatur findet sich kein Hinweis auf eine mögliche Erklärung.

Patienten mit Atemnot zeigten im Vergleich zu Patienten ohne Atemnot zusätzlich einen signifikant schlechteren Funktionsstatus. Dieses Ergebnis unterstreicht, dass es sich bei Patienten mit Atemnot um eine besonders schwer kranke Patientengruppe handelt, die besondere Aufmerksamkeit und Zuwendung benötigt.

Ho et al. (2001) und Zimmermann et al. (2010) konnten zeigen, dass zunehmende Atemnot in einem starken und statistisch signifikanten Zusammenhang mit einem abnehmenden Funktionsstatus steht. Auch Hayes et al. (2006) hatten in einer Analyse über 100 Krebspatienten einen signifikant schlechteren Funktionsstatus bei Patienten mit Atemnot festgestellt.

Anhand der vorliegenden Daten kann keine Aussage darüber getroffen werden, ob das Vorhandensein des Symptoms Atemnot einen schlechteren Funktionsstatus bedingt oder ob umgekehrt ein schlechter Funktionsstatus für ein häufigeres Auftreten von Atemnot verantwortlich ist. Dieses ist der Methode der Querschnittsanalyse zu schulden, mit der ausschließlich Assoziationen mit Atemnot beschrieben werden können. Zur Klärung von Kausalzusammenhängen wäre die Durchführung einer randomisierten kontrollierten Studie (RCT) unabdingbar.

Es sollte nicht außer Acht gelassen werden, dass für die vorliegende Arbeit ausschließlich Daten analysiert wurden, die im stationären Umfeld erhoben wurden. Bei der Betrachtung des Funktionsstatus der ausgewerteten Palliativpatienten zeigte sich, dass es sich insgesamt um eine sehr kranke Patientengruppe handelte. 75,0 \% aller Patienten hatten einen Funktionsstatus von 3 oder 4, was eine Bettlägerigkeit von mehr als $50 \%$ der Wachzeit bedeutete. Es ist wahrscheinlich, dass der Umstand, dass sich ein Patient in einer stationären Einrichtung befand, zu einem häufigeren Aufenthalt im Bett führte oder entsprechend von Ärzten bzw. Pflegenden wahrgenommen und im HOPE-Dokumentationsbogen beurteilt wurde. Insofern muss dieses Ergebnis im Rahmen stationärer Einrichtungen interpretiert werden und kann nicht ohne weiteres auf ambulante Patienten übertragen werden. An dem schlechteren Funktionsstatus der Patienten mit Atemnot im Vergleich zu den Patienten ohne Atemnot ändert das jedoch nichts. 
Symptomlast und Funktionsstatus sind (neben Coping-Strategien und sozialer Unterstützung) zwei Faktoren, die in hohem Maße die Lebensqualität von schwer kranken Patienten beeinflussen (McMillan und Small 2002, Moody und McMillan 2003).

Smith et al. (2001) ermittelten in ihrer Arbeit über 120 ambulante Bronchialkarzinompatienten eine schlechtere Lebensqualität für Patienten mit schwerer Atemnot. Henoch et al. (2007) zeigten eine Verschlechterung von Atemnot als auch der Lebensqualität im zeitlichen Verlauf bei 106 Patienten mit unheilbarem Bronchialkarzinom. In der Multiregressionsanalyse war Atemnot zwar kein signifikant bestimmender Faktor für die Lebensqualität, jedoch war die Ausprägung von Atemnot in dieser Patientengruppe eher gering.

\subsubsection{Prognose}

Die Einschätzung der Überlebenszeit eines Patienten gehört $\mathrm{zu}$ den wesentlichen und anspruchsvollsten Aufgaben in der Palliativmedizin (Glare und Sinclair 2008). Ihr kommt eine Schlüsselstellung zu, wenn Patienten, Angehörige und medizinisches Personal realistische Ziele setzen wollen und Entscheidungen am Ende des Lebens treffen müssen. Prognostische Einschätzungen, die auf klinischer Beobachtung und Erfahrung beruhten, erwiesen sich in Studien regelmäßig als unzuverlässig und zu optimistisch (Stiel et al. 2010 a, Christakis und Lamont 2000, Gripp et al. 2007). Um die Prognoseeinschätzung zu verbessern und damit die Kommunikation mit Schwerstkranken zu erleichtern, haben mehrere Arbeitsgruppen in der Vergangenheit versucht, verlässliche Punktesysteme zur Einschätzung von Überlebenszeit in der Palliativmedizin auszuarbeiten (Morita et al. 1999, Glare und Sinclair 2008). Stiel et al. (2010 a) fanden jedoch in ihrer Arbeit über die Aussagekraft von zwei prognostischen Beurteilungsinstrumenten (Palliative Prognostic Index (PPI) und Palliative Prognostic Score (PaP-S)) lediglich eine moderate Korrelation mit der Überlebenszeit.

Die Ergebnisse der vorliegenden Arbeit lassen vermuten, dass das Symptom Atemnot bei schwer kranken Patienten in einem Zusammenhang mit geringerer Überlebenszeit steht und als ein Risikofaktor für früheres Versterben angesehen werden kann. Dabei scheint allein das Vorhandensein von Atemnot die Prognose zu verschlechtern. Noch deutlicher zeigte sich diese Tendenz jedoch bei der Betrachtung der Atemnotintensitäten: Mit zunehmender Atemnot stieg das Risiko zu versterben und sank die Überlebenszeit.

Eine systematische Literaturrecherche von Maltoni et al. (2005) erbrachte ebenfalls einen starken Anhaltspunkt für die prognostische Signifikanz des Symptoms Atemnot bei Krebspatienten. Auch Nishimura et al. (2002) fanden in einer Studie bei 227 COPD-Patienten einen engen Zusammenhang zwischen Atemnotintensität und Überlebensrate. Weitere Publikationen bestätigen dieses (Vigano et al. 2000, Pirovano et al. 1999, Maltoni et al. 1995, Heyse-Moore et al. 1991, Vitetta et al. 2001, Teunissen et al. 2006).

Es konnte keine Studie gefunden werden, in der Atemnot nicht mit einer kürzeren Überlebenszeit korrelierte. Zwar kamen Cuervo Pinna et al. (2009) zu dem Schluss, keinen signifikanten 
Zusammenhang zwischen Atemnot und Überlebensrate feststellen zu können. Bei genauerer Analyse der Studiendaten ergab jedoch auch diese Arbeit, dass Patienten mit Atemnot eine kürzere Überlebenszeit aufwiesen als Patienten ohne Atemnot. Die „fehlende Signifikanz“ erklärt sich durch eine etwas fragwürdige Erzeugung der Atemnotgruppe, die sowohl Patienten mit initialer Atemnot als auch Patienten, die erst im stationären Verlauf Atemnot entwickelten, umfasste. Bei getrennter Analyse ergab sich für letztere Patientengruppe, die mit N=23 noch dazu eher klein war, eine längere Überlebenszeit. Patienten, die bei Aufnahme Atemnot angaben, zeigten jedoch eine deutlich kürzere Überlebenszeit im Vergleich zu Patienten ohne Atemnot. Für diesen Gruppenvergleich fehlte allerdings die Angabe einer Signifikanz. Insofern bestätigt die Arbeit von Cuervo Pinna et al. (2009) letztlich das Ergebnis der hier vorliegenden.

\subsubsection{Assoziation von Atemnot mit anderen Symptomen}

Diese Arbeit konnte zeigen, dass eine maligne Neubildung der Lunge, ein schlechter Funktionsstatus sowie die Symptome Schwäche und Angst in einem statistisch signifikanten Zusammenhang mit Atemnot bei schwer kranken Patienten stehen. Trotzdem erklärt das Modell in der Multiregressionsanalyse nur 5,9 bis 7,9\% der Atemnotvariation. Insofern scheinen viele weitere Einzelfaktoren, Einfluss auf das Gesamtempfinden des komplexen Symptoms Atemnot zu haben.

$\mathrm{Zu}$ einem ähnlichen Ergebnis kamen auch Bruera et al. (2000) in einer Untersuchung über Atemnot und zusammenhängende Faktoren bei 135 Patienten mit fortgeschrittenen Krebserkrankungen. Sie fanden in seiner multivariaten Analyse, dass maligne Erkrankungen der Lunge und Angst unabhängig voneinander mit der Stärke von Atemnot korrelierten. Für Schwäche konnte jedoch keine unabhängige Korrelation gezeigt werden. Auch dieses Modell konnte nur $18 \%$ der Varianz von Atemnot erklären. Dudgeon et al. (2001) konnten in einer Multiregressionsanalyse mit Hilfe der Risikofaktoren (predictors of dyspnoea) COPD, Asthma, Bestrahlung der Lunge, mediastinale Metastasen und Baumwollstaub nur 7,4 \% der Varianz von Atemnot begründen. Allein Henoch et al. (2007) fanden ein etwas aussagekräftigeres Modell mit einer Varianzerklärung von 21-35\%. Neben Angst, Depression, Müdigkeit, Husten und dem Funktionsstatus schien hier vor allem die Variable „,coping capacity“ (Bewältigungsfähigkeit) eine starke Auswirkung zu zeigen: Je niedriger die „,coping capacity“ desto höher das Risiko für Atemnot.

Walsh et al. (2000) fanden in einer multivariaten Analyse bei 1000 Patienten mit fortgeschrittenen Krebserkrankungen keinen Zusammenhang von Atemnot mit Alter, Geschlecht oder Funktionsstatus. In einer weiteren Analyse zu Symptomclustern bei dieser Patientengruppe (Walsh und Rybicki 2006) unter anderem ein ,aerodigestives Cluster“ identifiziert werden, welches ein gemeinsames Auftreten von Atemnot mit Husten, Heiserkeit und Schluckstörungen beschreibt. Kein gehäuftes Auftreten fand sich in dieser Analyse hingegen von Atemnot mit Angst oder Depression.

Insbesondere für Angst wird jedoch seit längerem ein Zusammenhang mit Atemnot diskutiert. Delgado-Guay et al. (2009) untersuchten 216 Patienten mit fortgeschrittenen Krebserkrankungen auf 
Depression und Angst und weitere Symptome. Sie fanden sowohl bei den Patienten mit Depression als auch bei solchen mit Angst eine signifikant höhere Atemnotprävalenz. Zusätzlich zeigte er eine moderate Korrelation zwischen Angst und Atemnot sowie eine schwache Korrelation zwischen Depression und Atemnot.

Dudgeon und Lertzman (1998) fanden nur eine schwache Korrelation zwischen Atemnot und Angst. Henoch et al. (2008) sahen die „coping capacity“ (Bewältigungsfähigkeit), den Funktionsstatus sowie die Symptome Angst, Depression, Müdigkeit und Husten mit bestimmten Qualitäten von Atemnot korreliert. Currow et al. (2009 b) beschrieben signifikante Assoziationen zwischen Atemnot und dem weiblichem Geschlecht, geringem Einkommen und höherem Lebensalter.

Zusammenfassend lässt sich sagen, dass starke Zusammenhänge zwischen Atemnot und weiteren Faktoren mit keiner der erwähnten Arbeiten zu belegen sind.

Trotz der Tatsache, dass bislang wenig Eindeutiges über Assoziation und Anhäufung von Atemnot mit anderen Merkmalen und Symptomen herausgefunden werden konnte und auch diese Arbeit keine wesentlichen neuen Erkenntnisse beisteuern kann, bleibt die weitere Suche nach Zusammenhängen eine wichtige Aufgabe zukünftiger Forschung. Die Behandlung eines Symptoms könnte positive wie negative Auswirkungen auf ein anderes zusammenhängendes Symptom haben. Ebenso könnten sich für zusammenhängende Symptome gemeinsame pathophysiologische Ursprungspfade zeigen. Das ließe hoffen, dass ein Medikament zur Behandlung eines Symptoms auch das zusammenhängende lindern könnte.

\subsubsection{Fremd- und Eigeneinschätzung}

Die Eigeneinschätzung von Symptomen durch den Patienten ist der direkteste und sicherste Weg, den wahren Zustand eines Patienten zu evaluieren. Der Einsatz dieser Methode ist jedoch bei schwer kranken Patienten nicht immer möglich. Inwieweit eine Fremdeinschätzung durch Angehörige oder professionelle Betreuer in diesen Situationen die Eigeneinschätzung ersetzen kann, wird seit langem kritisch hinterfragt. Einige Studien haben sich bereits in der Vergangenheit der Thematik angenommen, die Ergebnisse sind allerdings uneinheitlich (Stroemgren et al. 2001 a und b).

Die vorliegende Arbeit fand beim Vergleich der Fremd- mit der Eigeneinschätzung von 2.623 Patienten zum Vorhandensein beziehungsweise Fehlen des Symptoms Atemnot mit einer Sensitivität von $81,8 \%$ und einer Spezifität von 79,8\% eine relativ gute Vereinbarkeit. Auch der Grad der Übereinstimmung zeigte mit einem Cohen's $\kappa$ von 0,62 einen guten Wert.

Eine prospektive Studie von Oi-Ling et al. (2005) ergab - allerdings nur bei 30 Patienten - ebenfalls eine gute bzw. befriedigende Übereinstimmung in der Einschätzung des Symptoms Atemnot zwischen Pflegepersonal $(\kappa=0,65)$ bzw. Arzt $(\kappa=0,46)$ und Patient. 
In einer größeren Erhebung über 1.119 Patienten eines Akutkrankenhauses fand sich ebenfalls mit $\kappa=$ 0,46 ein befriedigender Wert bezüglich Atemnot beim Vergleich von Patientenaussage und Krankenakte (Pakhomov et al. 2008).

Im Gegensatz dazu ergab eine Studie von Nekolaichuk et al. (1999 b) eine vergleichsweise schlechte Übereinstimmung bei der Einschätzung von Atemnot.

Bei der Beurteilung der Atemnotintensität wurden in der vorliegenden Arbeit 34,3\% der Patienten falsch eingeschätzt. Dabei kam es etwa gleich häufig zu einer Über- bzw. Unterschätzung des Symptoms. Andere Studien hatten in der Vergangenheit häufiger Unterschätzungen durch den professionellen Betreuer beschrieben (Strömgren et al. 2001 a, Hayes et al. 2006, Stephens et al. 1997, Nekolaichuk et al. 1999 b, Zloklikovits et al. 2005).

Zusätzlich kamen Hayes et al. (2006) bzgl. der Übereinstimmung bei der Einschätzung der Atemnotintensität zu einem schlechten Ergebnis mit $\kappa=0,196$. Diese Untersuchung bezog sich allerdings nur auf 34 Patienten in einem Zentrum für Krebserkrankungen.

Im Vergleich zu allen anderen erhobenen Symptomen (Schmerz, Müdigkeit, Übelkeit, Verstopfung, Schwäche, Angst) wurde Atemnot in der vorliegenden Studie zuverlässiger fremdeingeschätzt. Eine Ursache für die vergleichsweise gute Übereinstimmung könnte sein, dass Atemnot von der überwiegenden Zahl der Patienten als quälendes Symptom empfunden und im Arzt-PatientenGespräch spontan erwähnt wird. Außerdem ist es möglich, dass Atemnot als häufiges bzw. relevantes Symptom von Arzt oder Pflegekraft in der täglichen Routine regelmäßiger abgefragt wird.

Auch die Studie von Oi-Ling et al. (2005) zeigte eine deutlich bessere Übereinstimmung in der Einschätzung von Atemnot (und Schmerz) im Vergleich zu Müdigkeit, Appetitlosigkeit und Kachexie. Ewing et al. (2006) beschrieben bei 109 Palliativpatienten insgesamt eine bessere Übereinstimmung bei der Einschätzung von körperlichen Symptomen im Vergleich zu psychischen. Dieses führten sie darauf zurück, dass sich körperliche Symptome deutlicher und damit beobachtbarer zeigten.

Die Untersuchung der Übereinstimmung bei Patienten mit unterschiedlichem Funktionsstatus zeigte in allen ECOG-Gruppen (außer ECOG 0, der wegen geringer Anzahl aber nicht aussagekräftig war) mit einem $\kappa>0,6$ eine gute Übereinstimmung zwischen der Fremd- und der Eigeneinschätzung bei der Einschätzung zum Vorhandensein des Symptoms Atemnot.

Dieses Ergebnis lässt hoffen, dass die Fremdeinschätzung durch einen professionellen Betreuer zumindest für das Symptom Atemnot auch bei Patienten mit schlechtem Funktionsstatus eine zuverlässige Alternative darstellen kann.

An dieser Stelle muss noch einmal darauf hingewiesen werden, dass die beschriebenen Daten, die dem Vergleich zu Grunde lagen, nur 49,9\% der Patienten des Ausgangskollektivs ausmachten, da nur für diese ein Selbsteinschätzungsbogen (MIDOS) vorlag. Die MIDOS-Gruppe umfasste Patienten, die 
tendenziell weniger morbide waren als das Ausgangskollektiv. Patienten der MIDOS-Gruppe hatten einen signifikant besseren Funktionsstatus, lagen seltener im Hospiz und der Anteil der Verstorbenen war geringer. Insbesondere aber waren MIDOS-Patienten seltener desorientiert als Patienten, bei denen keine Eigeneinschätzung vorlag. Diese Tatsache verzerrt die Daten insofern, als dass wache, ansprechbare und insgesamt weniger eingeschränkte Patienten leichter einschätzbar zu sein scheinen als schwerer kranke Patienten (Epstein et al. 1989).

Sneeuw et al. (1999) hatten hingegen in ihrer Arbeit bei 1.879 Vergleichen mit Hilfe der Dartmouth COOP Functional Health Assessment charts/WONCA unterschiedliche Grade der Übereinstimmung in Abhängigkeit vom Funktionsstatus gefunden. Seinen Ergebnissen zufolge ergaben sich die größten Unterschiede bei Patienten mit leicht- bzw. mittelgradig reduziertem Funktionsstatus.

Beim Vergleich der Einschätzung durch unterschiedliche Berufsgruppen erbrachte die vorliegende Arbeit insbesondere für Ärzte bzw. das Pflegepersonal keine Unterschiede. Beide Berufsgruppen zeigten mit $\kappa=0,62$ bzw. 0,61 eine vergleichbar gute Übereinstimmung mit der Eigeneinschätzung für das Vorhandensein von Atemnot und mit $\kappa=0,48$ bzw. 0,49 eine vergleichbar befriedigende Übereinstimmung für die Atemnotintensität.

Dieses Ergebnis steht nicht im Einklang mit früheren Arbeiten, die eine bessere Übereinstimmung bei der Fremdeinschätzung durch Pflegekräfte als bei Ärzten gezeigt hatten (Ewing et al. 2006, Nekolaichuk et al. 1999 a).

Zusammenfassend lässt sich sagen, dass die Eigeneinschätzung durch den Patienten der Goldstandard zur Beurteilung von Symptomen bei schwer kranken Patienten bleibt. Trotzdem deutet diese Analyse darauf hin, dass gerade für das Symptom Atemnot Fremdeinschätzungen durch einen professionellen Betreuer unabhängig von der Profession ein akzeptabler Ersatz bei mangelnder Möglichkeit der Eigeneinschätzung sein könnten - zumindest bei wachen Patienten. Die klinisch relevante Frage - ob eine Fremdeinschätzung des Symptoms Atemnot bei desorientierten oder komatösen Patienten valide ist - kann mit den Ergebnissen dieser Arbeit letztendlich auch nicht beantwortet werden, da diese Patientengruppe hier nicht untersucht wurde. Die Ergebnisse geben aber immerhin einen indirekten Hinweis (gute Übereinstimmung auch bei schlechtem Funktionsstatus) darauf, dass die Fremdeinschätzung bei fehlender Möglichkeit zur Selbsteinschätzung verlässlich ist. 


\subsection{Diskussion der Methodik}

\subsubsection{Registererfassung}

Die Datenerhebung für die vorliegende Arbeit erfolgte durch eine Registererfassung. Auf diese Art und Weise wurden zwischen 2006 und 2008 5.320 Patienten bei der Aufnahme in eine Einrichtung der spezialisierten Palliativversorgung in Deutschland dokumentiert und konnten der statistischen Datenanalyse zugeführt werden. So ergab sich eine ausreichend große Stichprobe, die eine hohe Aussagekraft über Charakteristika von stationären Palliativpatienten mit Atemnot zulässt.

Die Patienten stammten aus 26,1\% aller Einrichtungen der spezialisierten Palliativversorgung in Deutschland, was einen recht guten Überblick ermöglicht. Da allerdings sämtliche Palliativeinrichtungen in Deutschland zur Teilnahme an HOPE aufgerufen waren, kann bei dieser Antwortquote (response rate) ein systematischer Fehler (bias) nicht ausgeschlossen werden.

\subsubsection{Querschnittsanalyse}

Die vorliegende Arbeit benutzt außer bei der Betrachtung der Überlebenszeit ausschließlich Querschnittsdaten. Durch diese einzeitige Betrachtung erhält man einen guten Überblick über die Charakteristika von Palliativpatienten mit Atemnot zum Aufnahmezeitpunkt in eine Einrichtung der spezialisierten Palliativversorgung. Aussagen über den Verlauf dieser Patienten und insbesondere die Entwicklung der Atemnotsymptomatik können jedoch nicht getroffen werden und sollten Gegenstand zukünftiger Studien sein.

Mit der Methode der Querschnittsanalyse konnten lediglich Assoziationen mit Atemnot beschrieben werden. Zur Klärung von Kausalzusammenhängen wäre die Durchführung einer randomisierten kontrollierten Studie (RCT) unabdingbar. Realistisch gesehen wäre jedoch eine RCT mit ähnlich hohen Fallzahlen (N=5.320) kaum durchführbar.

Aufgrund der einzeitigen Erfassung und der Tatsache, dass Prädiktoren im Sinne der Kausalerfassung eine zumindest zweizeitige Erhebung benötigen, wurden die Multiregressionsanalyse sowie die Suche nach Prädiktoren zum Bestehen von Atemnot mit Vorsicht interpretiert.

\subsubsection{Spezialisierte Einrichtungen}

In die vorliegende Analyse wurden ausschließlich Patienten aus der stationären Palliativversorgung eingeschlossen. Dadurch war es möglich, sich auf ein relativ einheitliches Patientenkollektiv zu fokussieren, das in einer strukturell sehr ähnlichen Umgebung versorgt wurde, und eine bessere Vergleichbarkeit zu erreichen. Alle weiteren an HOPE teilnehmenden Einrichtungen (Onkologische Stationen, ambulante Dienste, ambulante Ärzte) wiesen große Unterschiede auf. 
Nachteil dieses Vorgehens ist allerdings, dass die gewonnenen Ergebnisse lediglich für den spezialisierten stationären Palliativbereich gelten und nicht ohne weiteres auf Patienten in der ambulanten Palliativversorgung übertragen werden dürfen.

\subsubsection{Fremdeinschätzung als Grundlage der Erfassung von Atemnot}

Bei der Einschätzung der Symptome insbesondere auch des Symptoms Atemnot stützt sich die vorliegende Analyse auf eine Fremdeinschätzung durch einen professionellen Betreuer. Dies ermöglichte den Einschluss aller Patienten aus der stationären Palliativversorgung aus dem Gesamtdatensatz von HOPE der Jahrgänge 2006 bis 2008. Wie man den Ergebnissen entnehmen kann, lagen nur für 49,9 \% aller Patienten ein MIDOS-Bogen und damit eine Selbsteinschätzung vor.

Da aber die Selbsteinschätzung durch den Patienten als der Gold-Standard in der Symptomeinschätzung gilt (Chan et al. 2004), limitiert dieses Vorgehen die Aussagekraft der Ergebnisse. Jedoch konnte in dieser Arbeit gezeigt werden, dass bei der Beurteilung des Symptoms Atemnot eine relativ gute Übereinstimmung zwischen Fremd- und Selbsteinschätzung vorliegt. Insofern scheint das Vorgehen, die Fremdeinschätzung eines Symptoms als Grundlage dieser Auswertung zuzulassen, zumindest für das Symptom Atemnot gerechtfertigt zu sein.

Dieses Vorgehen ermöglichte darüber hinaus den Einschluss von Patienten, die sich nicht mehr selbst einschätzen konnten, z.B. durch Desorientiertheit oder bei progredienter Vigilanzminderung in der Sterbephase. Denn häufig bleiben in Arbeiten, die sich ausschließlich auf eine Selbsteinschätzung stützen, Patienten mit verminderter kognitiver Leistungsfähigkeit unberücksichtigt. Die Folge ist eine eingeschränkte Wahrnehmung dieser Patienten mit Bewusstseinseinschränkungen und damit vor allem auch von schwerstkranken und sterbenden Patienten (Nauck 2003). Anhand der vorliegenden Daten ließ sich zeigen, dass Patienten mit einem schlechten Funktionsstatus in der Gruppe mit vorhandener Selbsteinschätzung (MIDOS) unterrepräsentiert waren. Insofern konnte man bei ausschließlicher Betrachtung der MIDOS-Gruppe mit einer Verzerrung der Daten durch insgesamt weniger stark eingeschränkte Patienten rechnen.

\subsubsection{Fragebogenerhebung}

Die Arbeit basiert auf einer Fragebogenerhebung, die in verschiedenen Institutionen von unterschiedlichen Personen vorgenommen wurde. In einer solchen Untersuchung fehlt die Möglichkeit, die Qualität der Datenerhebung zu kontrollieren. Das hatte zur Folge, dass Fehler oder Regelverstöße nicht ausgeschlossen werden konnten. Zusätzlich fand sich bei einigen Variablen ein nennenswerter Anteil an Fehldaten. Eine Verzerrung der Ergebnisse ist dadurch möglich. Konkret muss für die vorliegende Arbeit in diesem Zusammenhang die Variable „Verlauf“ mit einem Fehldatenanteil von $24,7 \%$ genannt werden. Für den überwiegenden Teil der Variablen lag der Fehldatenanteil jedoch erfreulicherweise immer $<5 \%$. 
Die mangelnde Qualitätskontrolle ist ein generelles Problem jeder Fragebogenerhebung, die von einer größeren Anzahl Personen vorgenommen wird. Nur auf diese Art und Weise konnte jedoch im Rahmen von HOPE ein solch großer Datensatz generiert werden. Zur Qualitätssicherung gab es neben Informationsveranstaltungen für die teilnehmenden Zentren für jeden Dokumentierenden ein Anleitungsschreiben mit detaillierten Instruktionen zum Ausfüllen der Fragebögen. Positiv könnte sich auch ausgewirkt haben, dass viele der partizipierenden Einrichtungen oftmals bereits seit Jahren an der HOPE-Dokumentation und -Qualitätssicherung teilgenommen hatten. Zusätzlich ist davon auszugehen, dass die große Fallzahl zumindest kleinere Ausreißer nivelliert. 


\subsection{Bedeutung}

\subsubsection{Bedeutung für die Praxis}

Atemnot ist ein häufiges Symptom bei Palliativpatienten. Die Ergebnisse dieser Arbeit zeigen, dass Patienten mit Atemnot im Vergleich zu anderen Patienten eine stärker belastete Patientengruppe darstellen. In der täglichen Praxis sollte Atemnot als Warnsignal für eine verstärkte Belastung verstanden werden, bei dem mit einem erhöhten Bedarf an palliativmedizinischen Maßnahmen zu rechnen ist.

Schwer kranke Patienten sollten aufgrund der hohen Prävalenz von Atemnot und der nicht immer eindeutigen Erkennbarkeit aktiv nach Vorhandensein und Schwere des Symptoms befragt werden. Dabei ist die subjektive Selbsteinschätzung durch den Patienten als Goldstandard anzusehen und sollte, wann immer möglich, durchgeführt werden. In den Fällen, in denen im klinischen Alltag eine Selbsteinschätzung von Atemnot nicht realisiert werden kann, ist der Fremdeinschätzung eine gewisse Zuverlässigkeit zuzuschreiben und ihre Anwendung sinnvoll.

Bei Vorliegen relevanter Atemnot ist aufgrund der zu erwartenden erhöhten Symptomlast die Erhebung und gegebenenfalls Behandlung weiterer körperlicher und psychischer Symptome unerlässlich. Ein besonderer Schwerpunkt sollte dabei auf der gezielten Suche nach häufig begleitenden psychischen Symptomen wie Angst und Depression liegen.

Atemnot muss als Risikofaktor für frühzeitiges Versterben angesehen werden. Dieser Punkt sollte Ärzten in der Therapieplanung und bei der Kommunikation mit Patienten und Angehörigen präsent sein, um realistische Ziele setzen zu können und gegebenenfalls rechtzeitige Entscheidungen am Lebensende für den Patienten und dessen Familie zu ermöglichen.

Der geringe Anteil an Patienten ohne Krebserkrankung in der vorliegenden Grundgesamtheit spiegelt die weltweite Situation in der stationären Palliativversorgung wider. Gerade aber diese Patienten litten häufiger unter Atemnot. Nicht-Tumor-Patienten einen uneingeschränkten Zugang zu palliativmedizinischen Diensten zu ermöglichen, bleibt somit eine wichtige Aufgabe für die Zukunft. Insgesamt stellen Diagnostik und Therapie des multifaktoriellen Symptoms Atemnot für Kliniker eine enorme Herausforderung dar. Jedoch brauchen gerade Patienten mit Atemnot aufgrund der offensichtlichen Schwere und Komplexität ihres Leidens eine effektive, umfassende Symptomkontrolle. Um diesem Anspruch gerecht werden zu können, ist der Einsatz eines multiprofessionellen Teams notwendig. Eine konsequente palliativmedizinische Fort- und Weiterbildung für alle Ärzte und Pflegekräfte auch außerhalb von Palliativeinrichtungen ist zusätzlich zu fordern. 


\subsubsection{Bedeutung für die Forschung}

Diese Arbeit beschreibt eine ausgeprägte Symptomlast von und damit eine große Anzahl von unkontrollierten Symptomen bei Patienten mit Atemnot. Eine effektive Symptomkontrolle zur Linderung ihres Leidens ist somit notwendig. Die derzeitigen Möglichkeiten hierfür sind jedoch begrenzt, da an pharmakologischen Maßnahmen bislang lediglich Opioide eine Evidenz für eine wirksame Linderung von Atemnot gezeigt haben (Jennings et al. 2001, Abernethy et al. 2003). Das Ziel zukünftiger Forschung muss die Entwicklung neuer Therapieverfahren auf dem pharmakologischen wie nicht-pharmakologischen Sektor sein. Zusätzlich ist die vermehrte Durchführung von randomisierten kontrollierten Studien zur Überprüfung der Wirkung von bereits bekannten und neuen Substanzen auf das Symptom Atemnot zu fordern. Um daneben das Verständnis der komplexen Pathophysiologie von Atemnot zu erweitern, ist intensivere Grundlagenforschung, unter anderem auf dem Gebiet des Neuroimagings notwendig (Leupoldt et al. 2009).

Trotz der Tatsache, dass sich bestimmte Faktoren ausmachen lassen, die mit dem gleichzeitigen Bestehen von Atemnot einhergehen, konnte mit der vorliegenden Arbeit kein konkretes Modell zur Erklärung der Varianz von Atemnot gefunden werden. Weitere Studien, die den Zusammenhang von Einflussfaktoren auf das komplexe Symptom Atemnot noch intensiver beleuchten und mögliche Therapieoptionen dieser Einzelfaktoren in ihrer Auswirkung auf das Symptom überprüfen, sollten durchgeführt werden. Diese Studien sollten prospektive Kohortenstudien sein, um einen zeitlich gerichteten Einfluss evaluieren zu können. Als wichtiger Einflussfaktor sollte hierbei u. a. auch die „,coping capacity“ (Bewältigunsfähigkeit) berücksichtigt werden.

Da die Selbsteinschätzung zwar weiterhin den Goldstandard bei der Einschätzung von Atemnot darstellt, bei Palliativpatienten aber häufig nicht durchführbar ist, besteht ein großer Bedarf an weiterer Erforschung und Entwicklung zuverlässiger Einschätzungsinstrumente für Schwerstkranke, um Falschbeurteilungen und damit Über- oder Unterbehandlungen zu verhindern. 


\section{Zusammenfassung}

Atemnot ist ein häufiges Symptom schwer kranker Patienten. Die hier vorgelegte Arbeit ist die erste deutschlandweite Erhebung, die Patienten mit dem Symptom Atemnot detailliert analysiert und beschreibt, und somit Grundlage für ein besseres Verständnis dieses komplexen und belastenden Symptoms.

In der vorliegenden Analyse litten 53,8 \% aller Patienten bei Aufnahme in eine Einrichtung der spezialisierten Palliativversorgung in Deutschland unter Atemnot in einer durchschnittlich mittelstarken Ausprägung. Diese Patienten hatten im Vergleich zu Patienten ohne Atemnot einen signifikant schlechteren Funktionsstatus und beklagten signifikant häufiger weitere körperliche und psychische Symptome in einer stärkeren Ausprägung. Zusätzlich zeigte diese Patientengruppe ein höheres Risiko früher zu versterben.

Die vorliegende Arbeit steht im Einklang mit den Ergebnissen großer internationaler Studien, die übereinstimmend feststellen, dass Patienten mit Atemnot ein besonders krankes und belastetes Patientenkollektiv mit einer schlechten Prognose darstellen.

Bestimmte Faktoren scheinen mit einem höheren Risiko für das gleichzeitige Bestehen von Atemnot einherzugehen. Ein konkretes Modell zur Erklärung der Varianz von Atemnot konnte jedoch mit den Daten dieser Arbeit nicht einwickelt werden.

Für die Prävalenz des Symptoms Atemnot gab es eine gute Übereinstimmung zwischen der Eigeneinschätzung durch den Patienten und der Fremdeinschätzung durch einen professionellen Betreuer. Allerdings waren die Ergebnisse bei der Einschätzung der Atemnotintensität nur befriedigend. Insgesamt bleibt die Eigeneinschätzung bei der Beurteilung von Symptomen weiterhin das Instrument der „Ersten Wahl“. Zumindest für das Symptom Atemnot kann aber auf eine Fremdeinschätzung durch einen Therapeuten zurückgegriffen werden, falls eine Eigeneinschätzung nicht durchführbar ist.

Eine wesentliche Stärke dieser Arbeit ist die große Anzahl von Patienten, die in die Analyse eingeschlossen werden konnte. Dieses ermöglicht eine gewisse Verallgemeinerung der Ergebnisse. Durch die Benutzung einer Fremdeinschätzung bei der Erfassung des Symptoms Atemnot konnten auch schwerst eingeschränkte und sterbende Patienten ausgewertet werden. Da jedoch bei einer Fremdeinschätzung die Kongruenz mit der Eigeneinschätzung je nach Symptom variiert, müssen die Ergebnisse mit einem gewissen Grad an Vorsicht interpretiert werden.

Diese Arbeit belegt, dass Patienten mit Atemnot ein besonders belastetes Patientenkollektiv darstellen, das besondere Aufmerksamkeit benötigt. Ziel einer palliativmedizinischen Betreuung muss eine effektive und umfassende Symptomkontrolle sein. Ein multiprofessionelles Team in der Versorgung sowie eine andauernde Fort- und Weiterbildung der betreuenden Kliniker scheinen dafür unabdingbar. Ziel zukünftiger Forschung muss die Entwicklung weiterer pharmakologischer und nichtpharmakologischer Therapien zur Linderung des Symptoms Atemnot sein. 


\section{Anhang}

\subsection{Basisbogen}

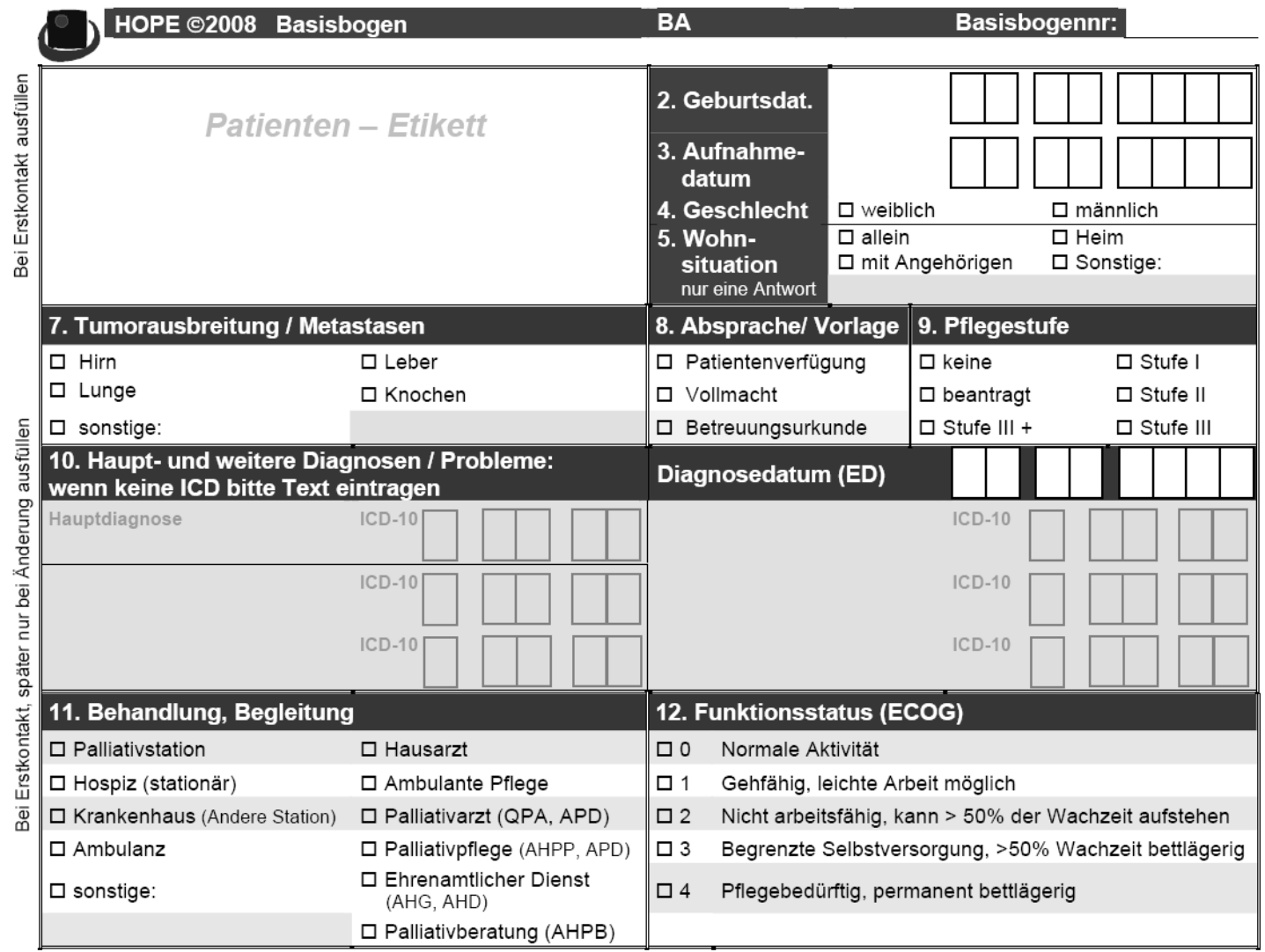

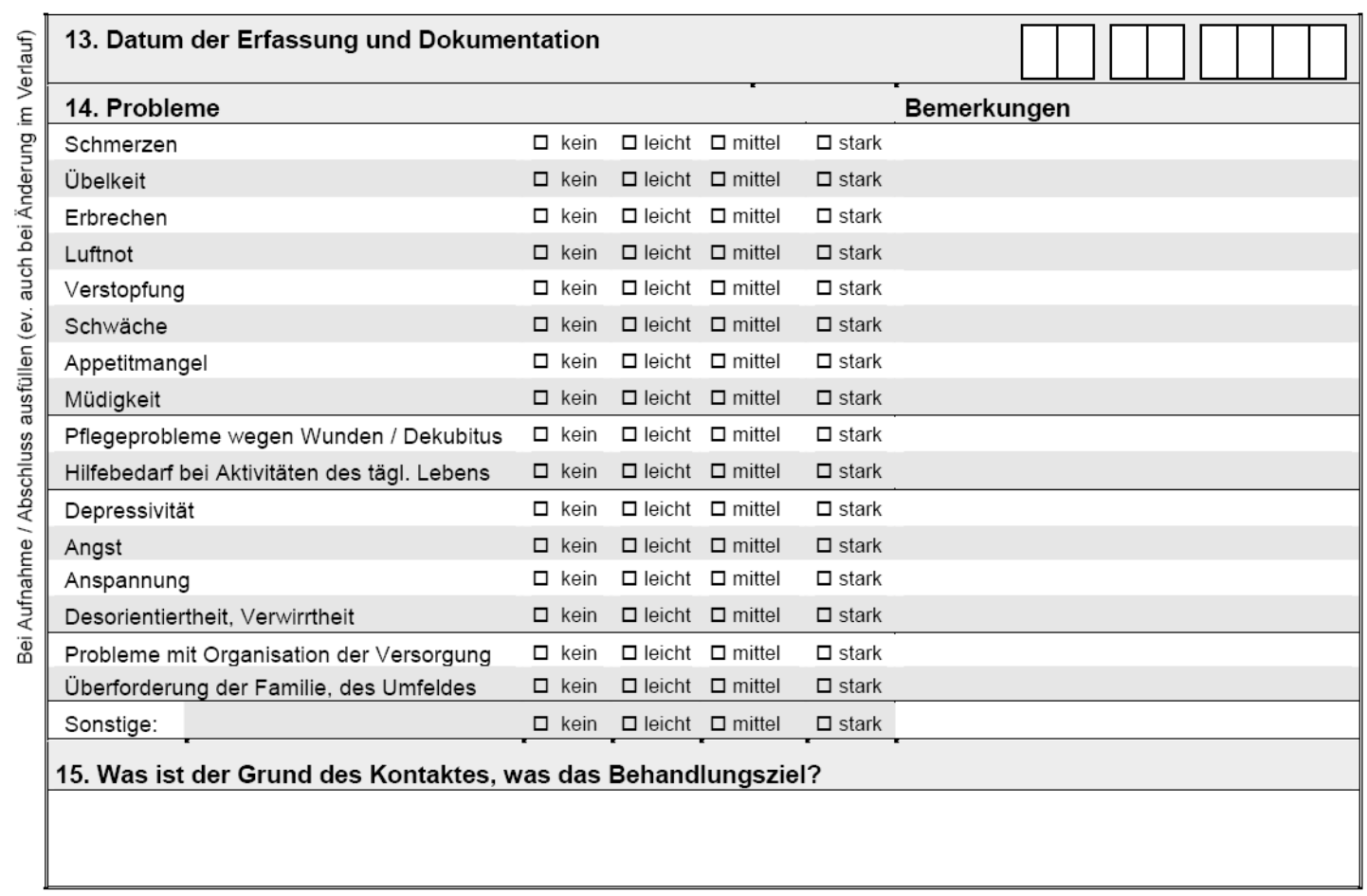




\begin{tabular}{|c|c|c|c|c|}
\hline \multicolumn{2}{|l|}{ 16. Medikation bis heute } & \multicolumn{3}{|l|}{ Medikamente } \\
\hline$\square$ Nichtopioide & $\square$ Sedativa / Anxiolytika & \multicolumn{3}{|l|}{ 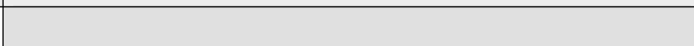 } \\
\hline$\square$ Opioide WHO-Stufe 2 & \multicolumn{4}{|l|}{$\square$ Magenschutz } \\
\hline$\square$ Opioide WHO-Stufe 3 & \multicolumn{4}{|l|}{$\square$ Laxanzien } \\
\hline$\square$ Koanalgetika & \multicolumn{4}{|l|}{$\square$ Antibiotika } \\
\hline$\square$ Kortikosteroide & \multicolumn{4}{|l|}{$\square$ Diuretika } \\
\hline$\square$ Antidepressiva & \multicolumn{4}{|l|}{$\square$ Kardiaka / Antihyperten. } \\
\hline$\square$ Antiemetika & \multicolumn{4}{|l|}{$\square$ Sonstige: } \\
\hline \multicolumn{5}{|l|}{$\square$ Neuroleptika } \\
\hline \multicolumn{5}{|c|}{ 17. Maßnahmen / Prozeduren Begleitung (bei Aufnahme bestehend und Abschluss) } \\
\hline Ehrenamtliche & \multicolumn{4}{|c|}{ Koordinatoren / Pflege / Arzt } \\
\hline$\square$ Unterstützung für Sterbende & $\square$ Palliativberatung & $\square$ enterale Ernährung & $\square$ & Chemotherapie \\
\hline$\square$ Unterstützung für Angehörige & $\square$ Koordination & $\square$ parenterale Ernährung & $\square$ & Strahlentherapie \\
\hline$\square$ Sozialanwaltschaftl. Handeln & $\square$ Psychische Stützung & $\square$ Medikamente sc & $\square$ & Transfusion \\
\hline $\begin{array}{l}\text { Auseinandersetzung in } \\
\text { Glaubens- und Lebensfragen }\end{array}$ & $\square$ Sozialrechtliche Beratung & $\square$ Medikamente iv: & $\square$ & Pleurapunktion \\
\hline $\begin{array}{l}\square \text { Hilfe bei Organisation } \\
\text { letzter Dinge }\end{array}$ & $\begin{array}{l}\square \text { Beratung in } \\
\text { ethischen Fragen. }\end{array}$ & $\begin{array}{l}\text { Medikamente epidural, } \\
\text { intrathekal }\end{array}$ & $\square$ & Aszitespunktion \\
\hline $\begin{array}{l}\square \text { Unterstützung in akuten } \\
\text { Notsituationen }\end{array}$ & $\square$ Angehörigen-Anleitung & $\square$ Anlage / Wechsel ZVK & & $\begin{array}{l}\text { Versorgung zentraler } \\
\text { Zugänge (ZVK, Port...) }\end{array}$ \\
\hline $\begin{array}{l}\square \text { Dasein } \\
\text { (Erleben von Gemeinsamkeit) }\end{array}$ & $\square$ Angehörigenbegleitung & $\square$ Anlage / Wechsel DK & $\square$ & $\begin{array}{l}\text { Versorgung Stomata } \\
\text { (Tracheo, Uro, Ileo, Colo...) }\end{array}$ \\
\hline $\begin{array}{l}\square \text { Sitzwachen } \\
\text { (z.B. im Sterbeprozess) }\end{array}$ & $\square$ Trauerbegleitung & $\begin{array}{l}\square \text { Multimodale } \\
\text { Schmerztherapie }\end{array}$ & $\square$ & Darmspülung \\
\hline \multirow[t]{4}{*}{$\square$ Sonstiges: } & $\square$ Case Management & $\square$ Physiotherapie & & $\begin{array}{l}\text { Lagerungsbehandlung } \\
\text { (path. Fraktur, Spezialbett...) }\end{array}$ \\
\hline & $\square$ Musiktherapie & $\square$ Psychotherapie & & $\begin{array}{l}\text { Wundversorgung } \\
\text { (Dekubitus, Fisteln, Tumor..) }\end{array}$ \\
\hline & $\square$ Kunsttherapie & $\square$ Sonstiges: & & \\
\hline & $\square$ Sonstiges: & & & \\
\hline \multicolumn{5}{|l|}{ besonderer Aufwand mit: } \\
\hline \multicolumn{5}{|c|}{ 18. Welches Problem konnte besonders gut gelöst werden? } \\
\hline \multicolumn{5}{|c|}{ 18a. Welches Problem konnte nicht ausreichend gelöst werden? } \\
\hline \multicolumn{5}{|c|}{ 19. Wer hat den Bogen ausgefüllt? } \\
\hline$\square$ Krankenpflege $\quad \square$ Arzt & $\square$ Ehrenamtl. & $\square$ Sozialarbeiter & $\square$ & Seelsorger $\square$ Anderer \\
\hline
\end{tabular}

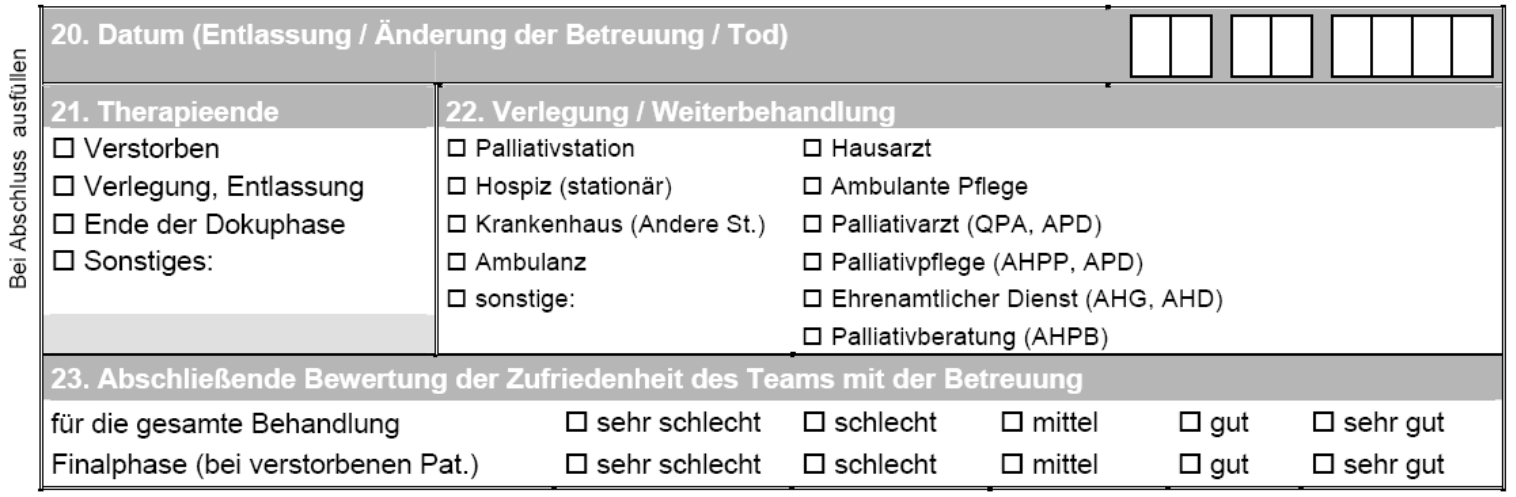

Abbildung 19: Basisbogen in der Version von 2008 


\subsection{MIDOS-Bogen}

Sehr geehrte Patientin, sehr geehrter Patient,

Sie kennen Ihre Situation selber am Besten. Darum bitten wir Sie, diesen Bogen sorgfältig auszufüllen und die Aussagen so anzukreuzen, wie Sie sie im

Augenblick bei sich selber wahrnehmen.

Vielen Dank für Ihre Mitarbeit!
1. IDNR

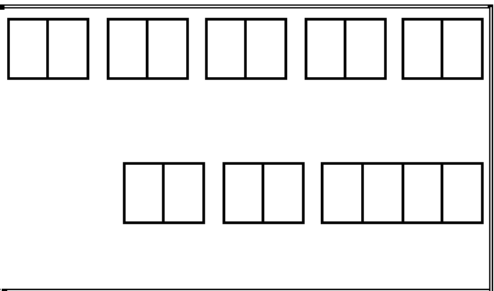

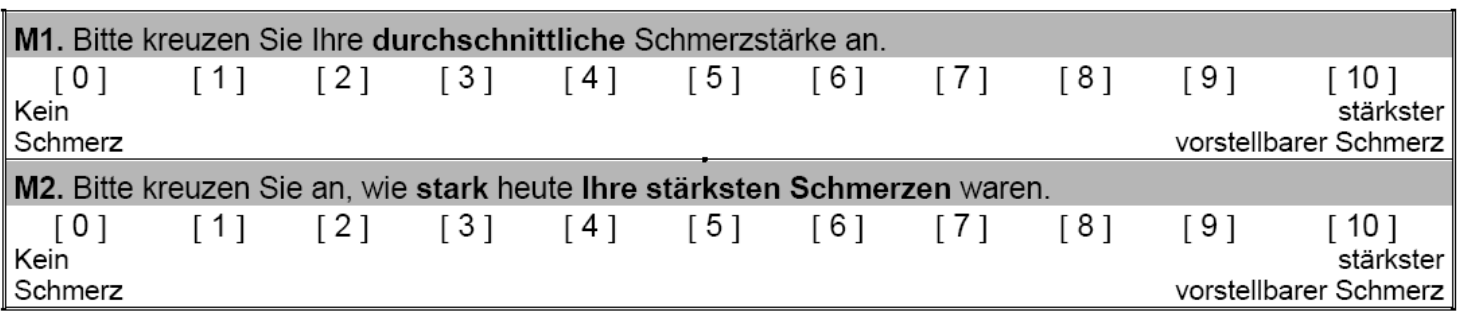

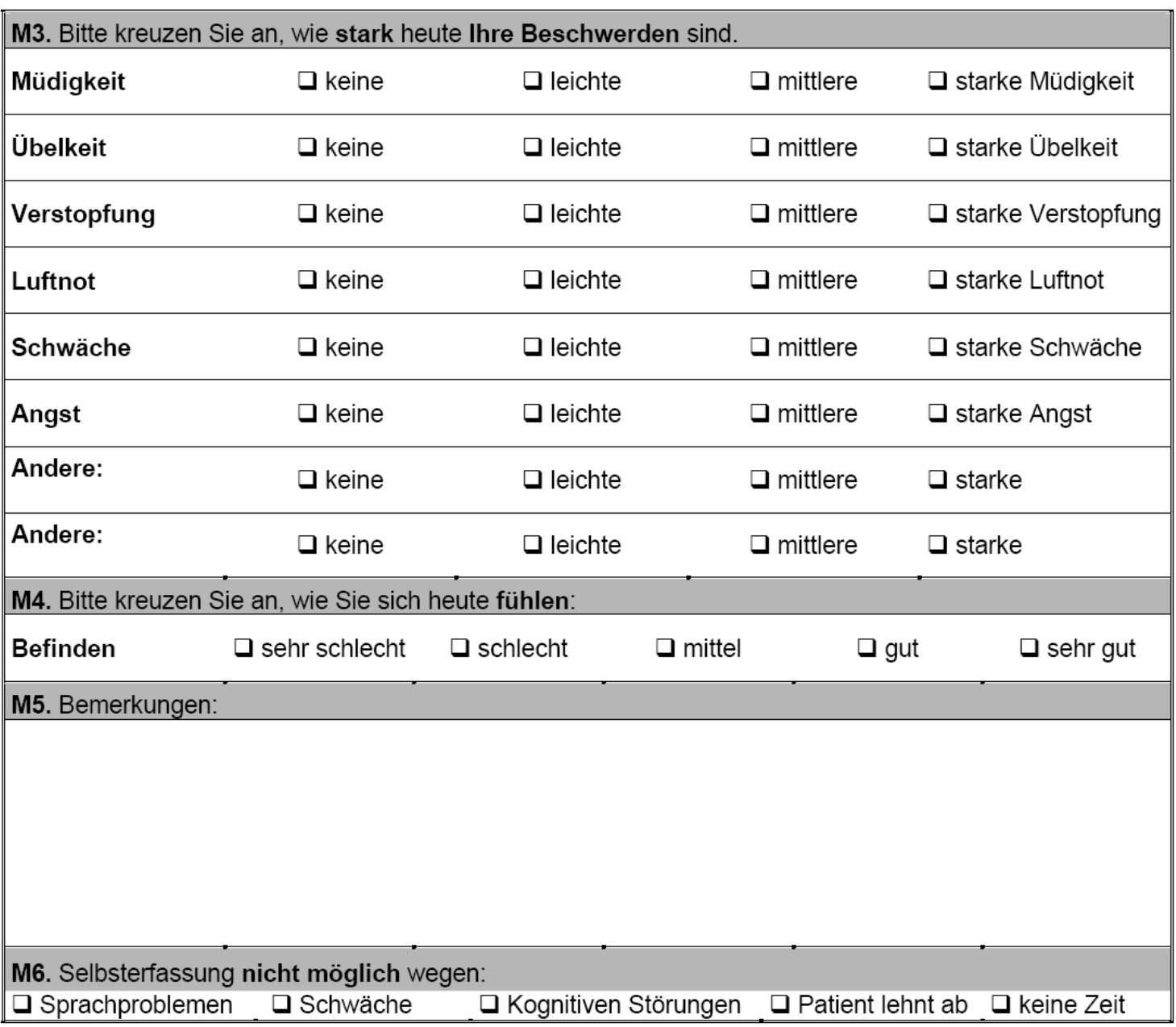

Abbildung 20: MIDOS-Bogen in der Version von 2008 


\subsection{Bestätigung der Ethikkommission}

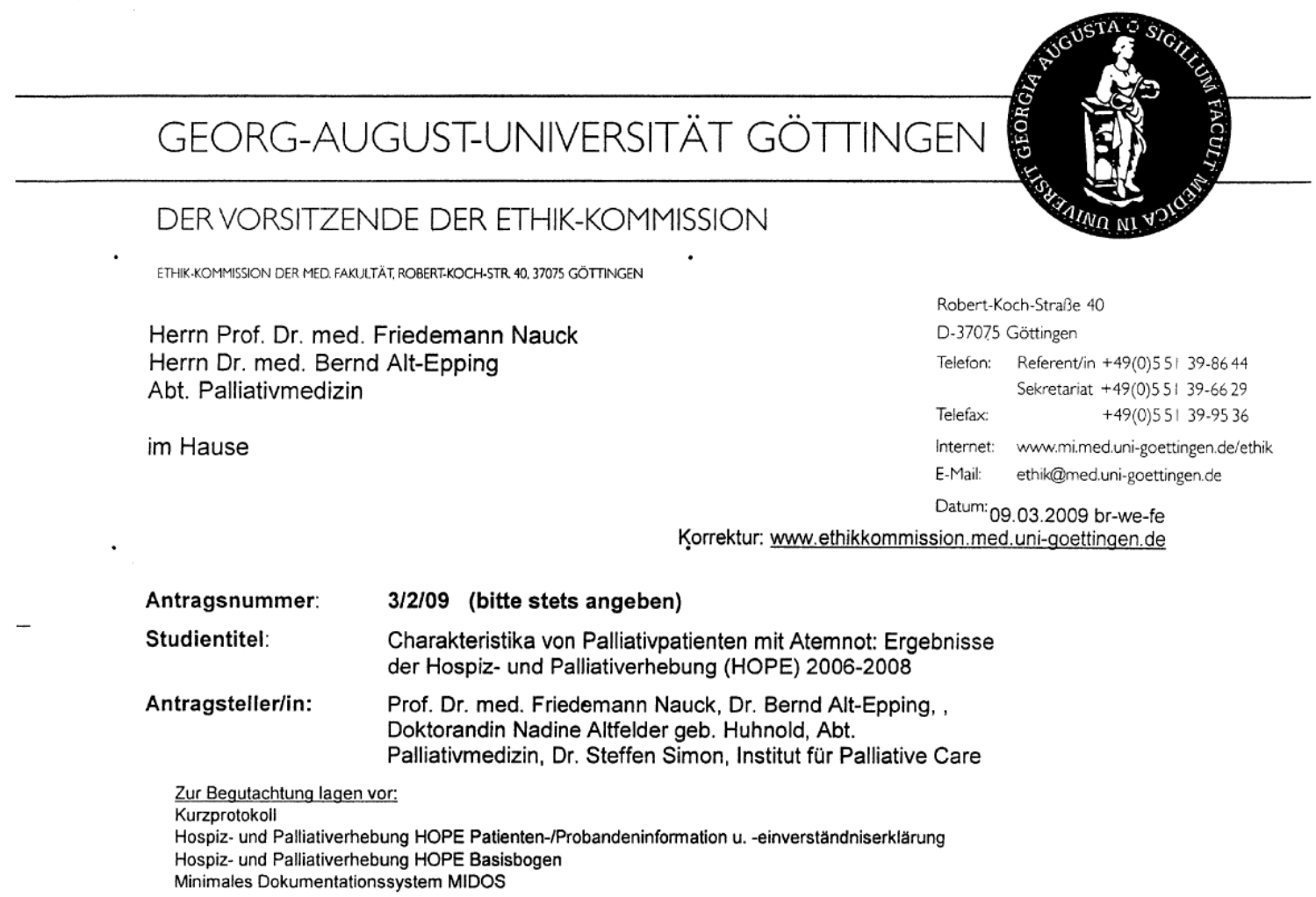

Sehr geehrter Herr Kollege Nauck, sehr geehrter Herr Kollege Alt-Epping,

die Ethik-Kommission der Medizinischen Fakultät der Universität Göttingen hat den oben genannten Antrag in der Sitzung vom 19.02.2009 beraten.

Die Ethik-Kommission hat keine ethischen oder rechtlichen Bedenken gegen das vorgelegte Studienvorhaben.

Wir möchten darauf hinweisen, dass möglicherweise das gewählte Kürzel "HOPE“ in der Hospiz- und Palliativ-Erfassung von einigen der potentiellen Beteiligten oder ihren Angehörigen als Sarkasmus verstanden werden könnte und bitten diesen Punkt noch einmal zu überdenken.

Wir wünschen Ihnen viel Erfolg bei der Durchführung Ihres Projektes.

Die Ethik-Kommission weist darauf hin, dass die ărztliche und juristische Verantwortung bei den jeweiligen Prüfärzten verbleibt. Auf die Einhaltung einschlägiger Gesetze und Rechtsvorschriften wird hingewiesen. Die nach Rechtslage notwendigen Unterrichtungen (u. A. Prüfplanänderungen, entsprechende Zwischenfallsereignisse, neue Datenlage, Nachmeldung von Prüfzentren, Abschlussbericht) sind der Ethik-Kommission unverzüglich vorzulegen. Die EthikKommission bestätigt, dass sie auf Grundlage nationaler Gesetze, Vorschriften sowie der GCP/ICH-Richtlinie arbeitet.

Mit freundlichen Grüßen

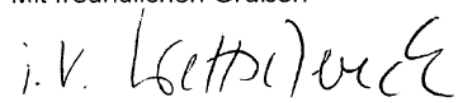

Prof. Dr. med. J. Brockmöller

Vorsitzender der Ethik-Kommission

Abbildung 21: Bestätigung der Ethikkommission 


\subsection{Weitere Ergebnistabellen}

\begin{tabular}{|l|c|}
\hline Symptome & $\begin{array}{l}\text { Prävalenz in der } \\
\text { Grundgesamtheit } \\
(\mathbf{\%})\end{array}$ \\
\hline Schmerz & 77,4 \\
Übelkeit & 49,5 \\
Erbrechen & 29,1 \\
Obstipation & 59,9 \\
Schwäche & 97,1 \\
Appetitlosigkeit & 85,0 \\
Müdigkeit & 91,8 \\
Depression & 59,9 \\
Angst & 66,8 \\
Anspannung & 73,5 \\
Desorientiertheit & 33,9 \\
\hline
\end{tabular}

Tabelle 51: Prävalenzen der Symptome des Basisbogens in der Grundgesamtheit 


\begin{tabular}{|c|c|c|c|}
\hline & Atemnot & keine Atemnot & $\mathbf{p}$ \\
\hline $\begin{array}{l}\text { Mammakarzinom } \\
(\%) \\
\text { Ja } \\
\text { Nein } \\
\text { Fehldaten } \\
\end{array}$ & $\begin{array}{l}304(10,6) \\
2242(78,4) \\
314(11,0)\end{array}$ & $\begin{array}{l}245(10,0) \\
1982(80,6) \\
233(9,5)\end{array}$ & 0,31 \\
\hline $\begin{array}{l}\text { kolorektales } \\
\text { Karzinom }(\%) \\
\text { Ja } \\
\text { Nein } \\
\text { Fehldaten } \\
\end{array}$ & $\begin{array}{l}271(9,5) \\
2275(79,5) \\
314(11,0)\end{array}$ & $\begin{array}{l}304(12,4) \\
1923(78,2) \\
233(9,5)\end{array}$ & 0,001 \\
\hline $\begin{array}{l}\text { Pankreaskarzinom } \\
(\%) \\
\mathrm{Ja} \\
\text { Nein } \\
\text { Fehldaten }\end{array}$ & $\begin{array}{l}145(5,1) \\
2401(84,0) \\
314(11,0)\end{array}$ & $\begin{array}{l}177(7,2) \\
2050(83,3) \\
233(11,0)\end{array}$ & 0,002 \\
\hline $\begin{array}{l}\text { Ovarialkarzinom } \\
(\%) \\
\text { Ja } \\
\text { Nein } \\
\text { Fehldaten } \\
\end{array}$ & $\begin{array}{l}103(3,6) \\
2443(85,4) \\
314(11,0) \\
\end{array}$ & $\begin{array}{l}89(3,6) \\
2138(86,9) \\
233(9,5) \\
\end{array}$ & 0,93 \\
\hline $\begin{array}{l}\text { Prostatakarzinom } \\
(\%) \\
\mathrm{Ja} \\
\text { Nein } \\
\text { Fehldaten }\end{array}$ & $\begin{array}{l}101(3,5) \\
2445(85,5) \\
314(11,0)\end{array}$ & $\begin{array}{l}134(5,4) \\
2093(85,1) \\
233(9,5)\end{array}$ & 0,001 \\
\hline $\begin{array}{l}\text { Karzinom des } \\
\text { MRR }(\%) \\
\text { Ja } \\
\text { Nein } \\
\text { Fehldaten } \\
\end{array}$ & $\begin{array}{l}89(3,1) \\
2457(85,9) \\
314(11,0) \\
\end{array}$ & $\begin{array}{l}72(2,9) \\
2155(87,6) \\
233(9,5) \\
\end{array}$ & 0,62 \\
\hline $\begin{array}{l}\text { Magenkarzinom } \\
(\%) \\
\mathrm{Ja} \\
\text { Nein } \\
\text { Fehldaten }\end{array}$ & $\begin{array}{l}86(3,0) \\
2460(86,0) \\
314(11,0)\end{array}$ & $\begin{array}{l}119(4,8) \\
2108(85,7) \\
233(9,5)\end{array}$ & 0,001 \\
\hline $\begin{array}{l}\text { Karzinom des LGS } \\
(\%) \\
\mathrm{Ja} \\
\text { Nein } \\
\text { Fehldaten }\end{array}$ & $\begin{array}{l}83(2,9) \\
2463(86,1) \\
314(11,0)\end{array}$ & $\begin{array}{l}92(3,7) \\
2135(86,8) \\
233(9,5)\end{array}$ & 0,11 \\
\hline $\begin{array}{l}\text { Nierenkarzinom } \\
(\%) \\
\mathrm{Ja} \\
\text { Nein } \\
\text { Fehldaten } \\
\end{array}$ & $\begin{array}{l}68(2,4) \\
2478(86,6) \\
314(11,0)\end{array}$ & $\begin{array}{l}77(3,1) \\
2150(87,4) \\
233(9,5)\end{array}$ & 0,11 \\
\hline
\end{tabular}

Tabelle 52: Vergleich der häufigsten Krebsdiagnosen von Patienten mit Atemnot bei Patienten mit und ohne Atemnot 


\begin{tabular}{|c|c|c|c|}
\hline & Atemnot & Keine Atemnot & $\mathbf{p}$ \\
\hline $\begin{array}{l}\text { Schmerz } \\
\text { Ja } \\
\text { Nein } \\
\text { Fehldaten } \\
\text { Kein } \\
\text { Leicht } \\
\text { Mittel } \\
\text { Stark } \\
\text { Fehldaten }\end{array}$ & $\begin{array}{l}2257(78,9) \\
580(20,3) \\
23(0,8) \\
580(20,3) \\
697(24,4) \\
889(31,1) \\
671(23,5) \\
23(0,8)\end{array}$ & $\begin{array}{l}1863(75,7) \\
586(23,8) \\
11(0,4) \\
586(23,8) \\
586(23,8) \\
674(27,4) \\
603(24,5) \\
11(0,4)\end{array}$ & 0,06 \\
\hline $\begin{array}{l}\text { Übelkeit } \\
\text { Ja } \\
\text { Nein } \\
\text { Fehldaten } \\
\text { Kein } \\
\text { Leicht } \\
\text { Mittel } \\
\text { Stark } \\
\text { Fehldaten }\end{array}$ & $\begin{array}{l}1476(51,6) \\
1343(47,0) \\
41(1,4) \\
1343(47,0) \\
698(24,4) \\
451(15,8) \\
327(11,4) \\
41(1,4)\end{array}$ & $\begin{array}{l}1158(47,1) \\
1282(52,1) \\
20(0,8) \\
1282(52,1) \\
525(21,3) \\
376(15,3) \\
257(10,4) \\
20(0,8)\end{array}$ & $\begin{array}{l}<0,001 \\
<0,001\end{array}$ \\
\hline $\begin{array}{l}\text { Erbrechen } \\
\text { Ja } \\
\text { Nein } \\
\text { Fehldaten } \\
\text { Kein } \\
\text { Leicht } \\
\text { Mittel } \\
\text { Stark } \\
\text { Fehldaten }\end{array}$ & $\begin{array}{l}854(29,9) \\
1963(68,6) \\
43(1,5) \\
1963(68,6) \\
356(12,4) \\
294(10,3) \\
204(7,1) \\
43(1,5)\end{array}$ & $\begin{array}{l}693(28,2) \\
1761(71,6) \\
6(0,2) \\
1761(71,6) \\
307(12,5) \\
217(8,8) \\
169(6,9) \\
6(0,2)\end{array}$ & $\mathbf{0 , 0 0 7}$ \\
\hline $\begin{array}{l}\text { Obstipation } \\
\text { Ja } \\
\text { Nein } \\
\text { Fehldaten } \\
\text { Kein } \\
\text { Leicht } \\
\text { Mittel } \\
\text { Stark } \\
\text { Fehldaten }\end{array}$ & $\begin{array}{l}1761(61,6) \\
1007(35,2) \\
92(3,2) \\
1007(35,2) \\
689(24,1) \\
650(22,7) \\
422(14,8) \\
92(3,2)\end{array}$ & $\begin{array}{l}1426(58,0) \\
971(39,5) \\
63(2,6) \\
971(39,5) \\
594(24,1) \\
515(20,9) \\
317(12,9) \\
63(2,6)\end{array}$ & $<0,001$ \\
\hline $\begin{array}{l}\text { Schwäche } \\
\text { Ja } \\
\text { Nein } \\
\text { Fehldaten } \\
\text { Kein } \\
\text { Leicht } \\
\text { Mittel } \\
\text { Stark } \\
\text { Fehldaten }\end{array}$ & $\begin{array}{l}2798(97,8) \\
29(1,0) \\
33(1,2) \\
\\
29(1,0) \\
181(6,3) \\
827(28,9) \\
1790(62,6) \\
33(1,2)\end{array}$ & $\begin{array}{l}2368(96,3) \\
66(2,7) \\
26(1,1) \\
66(2,7) \\
315(12,8) \\
867(35,2) \\
1186(48,2) \\
26(1,1)\end{array}$ & $<0,001$ \\
\hline
\end{tabular}




\begin{tabular}{|c|c|c|c|}
\hline $\begin{array}{l}\text { Appetitlosigkeit } \\
\text { Ja } \\
\text { Nein } \\
\text { Fehldaten } \\
\text { Kein } \\
\text { Leicht } \\
\text { Mittel } \\
\text { Stark } \\
\text { Fehldaten } \\
\end{array}$ & $\begin{array}{l}2500(87,4) \\
257(9,0) \\
103(3,6) \\
\\
257(9,0) \\
453(15,8) \\
728(25,5) \\
1319(46,1) \\
103(3,6) \\
\end{array}$ & $\begin{array}{l}2023(82,2) \\
349(14,2) \\
88(3,6) \\
\\
349(14,2) \\
455(18,5) \\
614(25,0) \\
954(38,8) \\
88(3,6) \\
\end{array}$ & $<0,001$ \\
\hline $\begin{array}{l}\text { Müdigkeit } \\
\text { Ja } \\
\text { Nein } \\
\text { Fehldaten } \\
\text { Kein } \\
\text { Leicht } \\
\text { Mittel } \\
\text { Stark } \\
\text { Fehldaten } \\
\end{array}$ & $\begin{array}{l}2661(93,0) \\
130(4,5) \\
69(2,4) \\
130(4,5) \\
525(18,4) \\
927(32,4) \\
1209(42,3) \\
69(2,4) \\
\end{array}$ & $\begin{array}{l}2223(90,4) \\
191(7,8) \\
46(1,9) \\
\\
191(7,8) \\
600(24,4) \\
810(32,9) \\
813(33,0) \\
46(1,9) \\
\end{array}$ & $<0,001$ \\
\hline $\begin{array}{l}\text { Depression } \\
\text { Ja } \\
\text { Nein } \\
\text { Fehldaten } \\
\text { Kein } \\
\text { Leicht } \\
\text { Mittel } \\
\text { Stark } \\
\text { Fehldaten } \\
\end{array}$ & $\begin{array}{l}1804(63,1) \\
864(30,2) \\
192(6,7) \\
\\
864(30,2) \\
854(29,9) \\
598(20,9) \\
352(12,3) \\
192(6,7) \\
\end{array}$ & $\begin{array}{l}1383(56,2) \\
920(37,4) \\
157(6,4) \\
\\
920(37,4) \\
683(27,8) \\
482(19,6) \\
218(8,9) \\
157(6,4) \\
\end{array}$ & $<0,001$ \\
\hline $\begin{array}{l}\text { Angst } \\
\text { Ja } \\
\text { Nein } \\
\text { Fehldaten } \\
\text { Kein } \\
\text { Leicht } \\
\text { Mittel } \\
\text { Stark } \\
\text { Fehldaten } \\
\end{array}$ & $\begin{array}{l}2048(71,6) \\
658(23,0) \\
154(5,4) \\
\\
658(23,0) \\
840(29,4) \\
755(26,4) \\
453(15,8) \\
154(5,4) \\
\end{array}$ & $\begin{array}{l}1506(61,2) \\
817(33,2) \\
137(5,6) \\
\\
817(33,2) \\
803(32,6) \\
483(19,6) \\
220(8,9) \\
137(5,6) \\
\end{array}$ & $<0,001$ \\
\hline $\begin{array}{l}\text { Anspannung } \\
\text { Ja } \\
\text { Nein } \\
\text { Fehldaten } \\
\text { Kein } \\
\text { Leicht } \\
\text { Mittel } \\
\text { Stark } \\
\text { Fehldaten } \\
\end{array}$ & $\begin{array}{l}2213(77,4) \\
493(17,2) \\
154(5,4) \\
\\
493(17,2) \\
877(30,7) \\
819(28,6) \\
517(18,1) \\
154(5,4) \\
\end{array}$ & $\begin{array}{l}1698(69,0) \\
637(25,9) \\
125(5,1) \\
\\
637(25,9) \\
828(33,7) \\
582(23,7) \\
288(11,7) \\
125(5,1) \\
\end{array}$ & $<0,001$ \\
\hline $\begin{array}{l}\text { Desorientiertheit } \\
\text { Ja } \\
\text { Nein } \\
\text { Fehldaten } \\
\text { Kein } \\
\text { Leicht } \\
\text { Mittel } \\
\text { Stark } \\
\text { Fehldaten } \\
\end{array}$ & $\begin{array}{l}957(33,5) \\
1777(62,1) \\
126(4,4) \\
\\
1777(62,1) \\
454(15,9) \\
299(10,5) \\
204(7,1) \\
126(4,4) \\
\end{array}$ & $\begin{array}{l}845(34,3) \\
1526(62,0) \\
89(3,6) \\
\\
1526(62,0) \\
339(13,8) \\
264(10,7) \\
242(9,8) \\
89(3,6) \\
\end{array}$ & 0,52 \\
\hline
\end{tabular}

Tabelle 53: Häufigkeiten der Symptome des Basisbogens bei Patienten mit und ohne Atemnot 


\begin{tabular}{|c|c|c|c|}
\hline & Atemnot & Keine Atemnot & $\mathbf{p}$ \\
\hline $\begin{array}{l}\text { Wundversorgung (\%) } \\
\text { Ja } \\
\text { Nein } \\
\text { Fehldaten } \\
\text { Kein } \\
\text { Leicht } \\
\text { Mittel } \\
\text { Stark } \\
\text { Fehldaten }\end{array}$ & $\begin{array}{l}1058(37,0) \\
1662(58,1) \\
140(4,9) \\
1662(58,1) \\
457(16,0) \\
364(12,7) \\
237(8,3) \\
140(4,9)\end{array}$ & $\begin{array}{l}811(33,0) \\
1532(62,3) \\
117(4,8) \\
\\
1532(62,3) \\
355(14,4) \\
253(10,3) \\
203(8,3) \\
117(4,8)\end{array}$ & 0,002 \\
\hline $\begin{array}{l}\text { Hilfe ADL (\%) } \\
\text { Ja } \\
\text { Nein } \\
\text { Fehldaten } \\
\text { Kein } \\
\text { Leicht } \\
\text { Mittel } \\
\text { Stark } \\
\text { Fehldaten }\end{array}$ & $\begin{array}{l}2657(92,9) \\
142(5,0) \\
61(2,1) \\
\\
142(5,0) \\
310(10,8) \\
733(25,6) \\
1614(56,4) \\
61(2,1)\end{array}$ & $\begin{array}{l}2190(89,0) \\
206(8,4) \\
64(2,6) \\
\\
206(8,4) \\
365(14,8) \\
631(25,7) \\
1194(48,5) \\
64(2,6)\end{array}$ & $<0,001$ \\
\hline $\begin{array}{l}\text { Versorgungsorganisation } \\
(\%) \\
\text { Ja } \\
\text { Nein } \\
\text { Fehldaten } \\
\text { Kein } \\
\text { Leicht } \\
\text { Mittel } \\
\text { Stark } \\
\text { Fehldaten }\end{array}$ & $\begin{array}{l}1853(64,8) \\
843(29,5) \\
164(5,7) \\
\\
843(29,5) \\
475(16,6) \\
629(22,0) \\
749(26,2) \\
164(5,7)\end{array}$ & $\begin{array}{l}1422(57,8) \\
883(35,9) \\
155(6,3) \\
883(35,9) \\
387(15,7) \\
473(19,2) \\
562(22,8) \\
155(6,3\end{array}$ & $<0,001$ \\
\hline $\begin{array}{l}\text { Familie (\%) } \\
\text { Ja } \\
\text { Nein } \\
\text { Fehldaten } \\
\text { Kein } \\
\text { Leicht } \\
\text { Mittel } \\
\text { Stark } \\
\text { Fehldaten }\end{array}$ & $\begin{array}{l}2115(74,0) \\
434(15,2) \\
311(10,9) \\
\\
434(15,2) \\
520(18,2) \\
688(24,1) \\
907(31,7) \\
311(10,9)\end{array}$ & $\begin{array}{l}1720(69,9) \\
478(19,4) \\
262(10,7) \\
\\
478(19,4) \\
461(18,7) \\
575(23,4) \\
684(27,8) \\
262(10,7\end{array}$ & $<0,001$ \\
\hline
\end{tabular}

Tabelle 54: Vergleich der Probleme von Patienten mit und ohne Atemnot 


\begin{tabular}{|c|c|c|c|}
\hline & Atemnot & Keine Atemnot & $\mathbf{p}$ \\
\hline $\begin{array}{l}\text { Nicht-Opioide } \\
\text { WHO I } \\
\text { Ja } \\
\text { Nein/ k. A. }\end{array}$ & $\begin{array}{l}1465(51,2) \\
1395(48,8)\end{array}$ & $\begin{array}{l}1289(52,4) \\
1171(47,6)\end{array}$ & 0,39 \\
\hline $\begin{array}{l}\text { WHO II } \\
\text { Ja } \\
\text { Nein/k. A. } \\
\end{array}$ & $\begin{array}{l}253(8,8) \\
2607(91,2) \\
\end{array}$ & $\begin{array}{l}201(8,2) \\
2259(91,8) \\
\end{array}$ & 0,38 \\
\hline $\begin{array}{l}\text { WHO III } \\
\text { Ja } \\
\text { Nein/k. A. }\end{array}$ & $\begin{array}{l}1798(62,9) \\
1062(37,1) \\
\end{array}$ & $\begin{array}{l}1544(62,8) \\
916(37,2)\end{array}$ & 0,94 \\
\hline $\begin{array}{l}\text { Koanalgetika } \\
\text { Ja } \\
\text { Nein/ k. A. }\end{array}$ & $\begin{array}{l}514(18,0) \\
2346(82,0)\end{array}$ & $\begin{array}{l}490(19,9) \\
1970(80,1)\end{array}$ & 0,07 \\
\hline $\begin{array}{l}\text { Steroide } \\
\text { Ja } \\
\text { Nein/k.A. }\end{array}$ & $\begin{array}{l}937(32,8) \\
1923(67,2) \\
\end{array}$ & $\begin{array}{l}742(30,2) \\
1718(69,8) \\
\end{array}$ & 0,04 \\
\hline $\begin{array}{l}\text { Antidepressiva } \\
\text { Ja } \\
\text { Nein/ k. A. }\end{array}$ & $\begin{array}{l}502(17,6) \\
2358(82,4)\end{array}$ & $\begin{array}{l}486(19,8) \\
1974(80,2)\end{array}$ & 0,04 \\
\hline $\begin{array}{l}\text { Antiemetika } \\
\text { Ja } \\
\text { Nein/k. A. }\end{array}$ & $\begin{array}{l}921(32,2) \\
1939(67,8) \\
\end{array}$ & $\begin{array}{l}842(34,2) \\
1618(65,8) \\
\end{array}$ & 0,12 \\
\hline $\begin{array}{l}\text { Neuroleptika } \\
\text { Ja } \\
\text { Nein/ k. A. }\end{array}$ & $\begin{array}{l}313(10,9) \\
2547(89,1)\end{array}$ & $\begin{array}{l}332(13,5) \\
2128(86,5)\end{array}$ & 0,005 \\
\hline $\begin{array}{l}\text { Sedativa/Anxiolytika } \\
\text { Ja } \\
\text { Nein/ k. A. }\end{array}$ & $\begin{array}{l}792(27,7) \\
2068(72,3) \\
\end{array}$ & $\begin{array}{l}609(24,8) \\
1851(75,2) \\
\end{array}$ & 0,02 \\
\hline $\begin{array}{l}\text { Magenschutz } \\
\text { Ja } \\
\text { Nein/k.A. }\end{array}$ & $\begin{array}{l}1323(46,3) \\
1537(53,7) \\
\end{array}$ & $\begin{array}{l}1133(46,1) \\
1327(53,9)\end{array}$ & 0,88 \\
\hline $\begin{array}{l}\text { Laxanzien } \\
\mathrm{Ja} \\
\mathrm{Nein} / \mathrm{k} \text {. A. }\end{array}$ & $\begin{array}{l}1040(36,4) \\
1820(63,6) \\
\end{array}$ & $\begin{array}{l}917(37,3) \\
1543(62,7)\end{array}$ & 0,49 \\
\hline $\begin{array}{l}\text { Antibiotika } \\
\text { Ja } \\
\text { Nein/ k. A. }\end{array}$ & $\begin{array}{l}535(18,7) \\
2325(81,3)\end{array}$ & $\begin{array}{l}275(11,2) \\
2185(88,8)\end{array}$ & $<0,001$ \\
\hline $\begin{array}{l}\text { Diuretika } \\
\text { Ja } \\
\text { Nein/k. A. }\end{array}$ & $\begin{array}{l}954(33,4) \\
1906(66,6)\end{array}$ & $\begin{array}{l}567(23,0) \\
1893(77,0)\end{array}$ & $<0,001$ \\
\hline $\begin{array}{l}\text { Kardiaka/ } \\
\text { Antihypertensiva } \\
\text { Ja } \\
\text { Nein/ k. A. }\end{array}$ & $\begin{array}{l}865(30,2) \\
1995(69,8)\end{array}$ & $\begin{array}{l}647(26,3) \\
1813(73,7)\end{array}$ & 0,002 \\
\hline
\end{tabular}

Tabelle 55: Vergleich der Medikamente von Patienten mit und ohne Atemnot bei Aufnahme in eine spezialisierte stationäre Palliativeinrichtung 


\begin{tabular}{|c|c|c|c|}
\hline & Atemnot & Keine Atemnot & $\mathbf{p}$ \\
\hline $\begin{array}{l}\text { Enterale Ernährung } \\
\text { Ja } \\
\text { Nein / k. A. }\end{array}$ & $\begin{array}{l}458(16,0) \\
2402(84,0)\end{array}$ & $\begin{array}{l}374(15,2) \\
2086(84,8)\end{array}$ & 0,42 \\
\hline $\begin{array}{l}\text { Parenterale } \\
\text { Ernährung } \\
\text { Ja } \\
\text { Nein / k. A. }\end{array}$ & $\begin{array}{l}473(16,5) \\
2387(83,5)\end{array}$ & $\begin{array}{l}365(14,8) \\
2095(85,2)\end{array}$ & 0,09 \\
\hline $\begin{array}{l}\text { Chemotherapie } \\
\text { Ja } \\
\text { Nein / k. A. }\end{array}$ & $\begin{array}{l}156(5,5) \\
2704(94,5)\end{array}$ & $\begin{array}{l}105(4,3) \\
2355(95,7)\end{array}$ & 0,05 \\
\hline $\begin{array}{l}\text { Transfusion } \\
\text { Ja } \\
\text { Nein / k. A. }\end{array}$ & $\begin{array}{l}157(5,5) \\
2703(94,5)\end{array}$ & $\begin{array}{l}128(5,2) \\
2332(94,8)\end{array}$ & 0,64 \\
\hline $\begin{array}{l}\text { Pleurapunktion } \\
\text { Ja } \\
\text { Nein / k. A. }\end{array}$ & $\begin{array}{l}149(5,2) \\
2711(94,8)\end{array}$ & $\begin{array}{l}14(0,6) \\
2446(99,4)\end{array}$ & $<0,001$ \\
\hline $\begin{array}{l}\text { Aszitespunktion } \\
\text { Ja } \\
\text { Nein / k. A. }\end{array}$ & $\begin{array}{l}128(4,5) \\
2732(95,5)\end{array}$ & $\begin{array}{l}70(2,8) \\
2390(97,2)\end{array}$ & 0,002 \\
\hline $\begin{array}{l}\text { Med. e. d./i. th. } \\
\text { Ja } \\
\text { Nein / k. A. }\end{array}$ & $\begin{array}{l}20(0,7) \\
2840(99,3)\end{array}$ & $\begin{array}{l}28(1,1) \\
2432(98,9) \\
\end{array}$ & 0,09 \\
\hline $\begin{array}{l}\text { ZVK-Versorgung } \\
\text { Ja } \\
\text { Nein / k. A. } \\
\end{array}$ & $\begin{array}{l}484(16,9) \\
2376(83,1)\end{array}$ & $\begin{array}{l}391(15,9) \\
2069(84,1)\end{array}$ & 0,31 \\
\hline $\begin{array}{l}\text { Multimodale } \\
\text { Schmerztherapie } \\
\text { Ja } \\
\text { Nein / k. A. } \\
\end{array}$ & $\begin{array}{l}578(20,2) \\
2282(79,8)\end{array}$ & $\begin{array}{l}505(20,5) \\
1955(79,5)\end{array}$ & 0,77 \\
\hline $\begin{array}{l}\text { Physiotherapie } \\
\text { Ja } \\
\text { Nein / k. A. }\end{array}$ & $\begin{array}{l}502(17,6) \\
2358(82,4)\end{array}$ & $\begin{array}{l}445(18,1) \\
2015(81,9)\end{array}$ & 0,61 \\
\hline $\begin{array}{l}\text { Lagerung } \\
\text { Ja } \\
\text { Nein / k. A. }\end{array}$ & $\begin{array}{l}442(15,5) \\
2418(84,5)\end{array}$ & $\begin{array}{l}331(13,5) \\
2129(86,5)\end{array}$ & 0,04 \\
\hline $\begin{array}{l}\text { Wundversorgung } \\
\text { Ja } \\
\text { Nein / k. A. }\end{array}$ & $\begin{array}{l}337(11,8) \\
2523(88,2)\end{array}$ & $\begin{array}{l}315(12,8) \\
2145(87,2)\end{array}$ & 0,26 \\
\hline $\begin{array}{l}\text { Psychotherapie } \\
\text { Ja } \\
\text { Nein / k. A. }\end{array}$ & $\begin{array}{l}188(6,6) \\
2672(93,4)\end{array}$ & $\begin{array}{l}155(6,3) \\
2305(93,7)\end{array}$ & 0,69 \\
\hline \multicolumn{4}{|l|}{$\begin{array}{l}\text { Nur 2007 und 2008: } \\
N=3459\end{array}$} \\
\hline $\begin{array}{l}\text { Med. s. c. } \\
\text { Ja } \\
\text { Nein / k. A. }\end{array}$ & $\begin{array}{l}536(28,9) \\
1320(71,1) \\
\end{array}$ & $\begin{array}{l}428(26,7) \\
1175(73,3) \\
\end{array}$ & 0,15 \\
\hline $\begin{array}{l}\text { Med. i. v. } \\
\text { Ja } \\
\text { Nein / k. A. }\end{array}$ & $\begin{array}{l}667(35,9) \\
1189(64,1)\end{array}$ & $\begin{array}{l}442(27,6) \\
1161(72,4)\end{array}$ & $<0,001$ \\
\hline $\begin{array}{l}\text { Strahlentherapie } \\
\text { Ja } \\
\text { Nein / k. A. }\end{array}$ & $\begin{array}{l}68(3,7) \\
1788(96,3)\end{array}$ & $\begin{array}{l}65(4,1) \\
1538(95,9)\end{array}$ & 0,55 \\
\hline $\begin{array}{l}\text { Angehörigenbetreuung } \\
\text { Ja } \\
\text { Nein / k. A. }\end{array}$ & $\begin{array}{l}837(45,1) \\
1019(54,9) \\
\end{array}$ & $\begin{array}{l}711(44,4) \\
892(55,6) \\
\end{array}$ & 0,66 \\
\hline
\end{tabular}

Tabelle 56: Vergleich der Maßnahmen und Therapien bei Patienten mit und ohne Atemnot 


\begin{tabular}{|c|c|c|c|c|c|}
\hline \multirow[t]{2}{*}{ Kategorie } & \multirow[t]{2}{*}{$\mathbf{N}$} & \multicolumn{2}{|c|}{$\begin{array}{c}\text { Korrelation mit dem } \\
\text { Vorhandensein von Atemnot }\end{array}$} & \multicolumn{2}{|c|}{$\begin{array}{c}\text { Korrelation mit der } \\
\text { Intensität von Atemnot }\end{array}$} \\
\hline & & Spearman's rho & $\mathbf{p}$ & Spearman's rho & p \\
\hline Alter & 5204 & $-0,016$ & 0,24 & $-0,023$ & 0,09 \\
\hline Altersgruppen & 5204 & $-0,027$ & 0,055 & $-0,035$ & 0,012 \\
\hline $\begin{array}{l}\text { Männliches } \\
\text { Geschlecht }\end{array}$ & 5259 & 0,044 & 0,001 & 0,038 & 0,006 \\
\hline Allein lebend & 3242 & $-0,017$ & 0,34 & $-0,013$ & 0,45 \\
\hline ECOG & 4986 & 0,088 & $<0,001$ & 0,102 & $<0,001$ \\
\hline Krebserkrankung & 5303 & $-0,030$ & 0,03 & $-0,032$ & 0,02 \\
\hline $\begin{array}{c}\text { Bösartige } \\
\text { Neubildung in der } \\
\text { Lunge }\end{array}$ & 5320 & 0,237 & $<0,001$ & 0,275 & $<0,001$ \\
\hline Bronchialkarzinom & 4773 & 0,198 & $<0,001$ & 0,227 & $<0,001$ \\
\hline Mammakarzinom & 4773 & 0,015 & 0,31 & 0,025 & 0,09 \\
\hline $\begin{array}{l}\text { kolorektales } \\
\text { Karzinom }\end{array}$ & 4773 & $-0,046$ & 0,001 & $-0,059$ & $<0,001$ \\
\hline Pankreaskarzinom & 4773 & $-0,045$ & $\mathbf{0 , 0 0 2}$ & $-0,052$ & $<0,001$ \\
\hline Ovarialkarzinom & 4773 & 0,001 & 0,93 & $-0,014$ & 0,318 \\
\hline Prostatakarzinom & 4773 & $-0,047$ & $\mathbf{0 , 0 0 1}$ & $-0,052$ & $<0,001$ \\
\hline Karzinom des MRR & 4773 & 0,007 & 0,62 & $-0,011$ & 0,44 \\
\hline Magenkarzinom & 4773 & $-0,048$ & $\mathbf{0 , 0 0 1}$ & $-0,052$ & $<0,001$ \\
\hline Karzinom des LGS & 4773 & $-0,023$ & 0,11 & $-0,030$ & 0,04 \\
\hline Nierenkarzinom & 4773 & $-0,023$ & 0,11 & $-0,021$ & 0,14 \\
\hline Metastasen & 5320 & 0,052 & $<0,001$ & 0,051 & $<0,001$ \\
\hline Hirn-Met. & 5320 & $-0,018$ & 0,20 & $-0,028$ & 0,04 \\
\hline Leber-Met. & 5320 & 0,012 & 0,36 & $-0,006$ & 0,66 \\
\hline Lungen-Met. & 5320 & 0,192 & $<0,001$ & 0,226 & $<0,001$ \\
\hline Knochen-Met. & 5320 & 0,009 & 0,53 & 0,006 & 0,65 \\
\hline Pleura-Met. & 5320 & 0,106 & $<0,001$ & 0,130 & $<0,001$ \\
\hline LK-Met. & 5320 & 0,062 & $<0,001$ & 0,076 & $<0,001$ \\
\hline Peritonealkarzinose & 5320 & $-0,022$ & 0,12 & $-0,040$ & 0,004 \\
\hline COPD & 5303 & 0,048 & $<0,001$ & 0,071 & $<0,001$ \\
\hline
\end{tabular}




\begin{tabular}{|c|c|c|c|c|c|}
\hline CHF & 5303 & 0,029 & 0,03 & 0,039 & 0,004 \\
\hline Schmerz & 5286 & 0,042 & 0,002 & 0,029 & 0,04 \\
\hline Schmerz-I & 5286 & 0,023 & 0,09 & 0,007 & 0,59 \\
\hline Übelkeit & 5259 & 0,049 & $<0,001$ & 0,029 & 0,04 \\
\hline Übelkeit-I & 5259 & 0,042 & 0,002 & 0,020 & 0,15 \\
\hline Erbrechen & 5271 & 0,023 & 0,10 & 0,000 & 0,99 \\
\hline Erbrechen-I & 5271 & 0,024 & 0,08 & $-0,001$ & 0,92 \\
\hline Obstipation & 5165 & 0,042 & 0,002 & 0,040 & 0,004 \\
\hline Obstipation-I & 5165 & 0,047 & 0,001 & 0,046 & 0,001 \\
\hline Schwäche & 5261 & 0,063 & $<0,001$ & 0,060 & $<0,001$ \\
\hline Schwäche-I & 5261 & 0,161 & $<0,001$ & 0,180 & $<0,001$ \\
\hline Appetitlosigkeit & 5129 & 0,083 & $<0,001$ & 0,089 & $<0,001$ \\
\hline Appetitlosigkeit-I & 5129 & 0,097 & $<0,001$ & 0,104 & $<0,001$ \\
\hline Müdigkeit & 5205 & 0,067 & $<0,001$ & 0,064 & $<0,001$ \\
\hline Müdigkeit-I & 5205 & 0,119 & $<0,001$ & 0,130 & $<0,001$ \\
\hline Depression & 4971 & 0,079 & $<0,001$ & 0,072 & $<0,001$ \\
\hline Depression-I & 4971 & 0,082 & $<0,001$ & 0,079 & $<0,001$ \\
\hline Angst & 5029 & 0,119 & $<0,001$ & 0,130 & $<0,001$ \\
\hline Angst-I & 5029 & 0,159 & $<0,001$ & 0,191 & $<0,001$ \\
\hline Anspannung & 5041 & 0,108 & $<0,001$ & 0,119 & $<0,001$ \\
\hline Anspannung-I & 5041 & 0,139 & $<0,001$ & 0,175 & $<0,001$ \\
\hline Desorientiertheit & 5105 & $-0,007$ & 0,63 & $-0,016$ & 0,26 \\
\hline Desorientiertheit-I & 5105 & $-0,019$ & 0,18 & 0,026 & 0,06 \\
\hline Symptomlast & 5320 & 0,321 & $<0,001$ & 0,292 & $<0,001$ \\
\hline Intensitätslast & 5320 & 0,289 & $<0,001$ & 0,319 & $<0,001$ \\
\hline Liegedauer & 4488 & $-0,105$ & $<0,001$ & $-0,133$ & $<0,001$ \\
\hline Überleben & 4008 & $-0,118$ & $<0,001$ & $-0,136$ & $<0,001$ \\
\hline
\end{tabular}

Tabelle 57: Korrelationen ausgewählter Merkmale mit dem Vorhandensein bzw. der Intensität von Atemnot 


\section{Literatur}

Abernethy AP, Wheeler JL (2008): Total dyspnoea. Curr Opin Support Palliat Care 2(2), 110-113

Abernethy AP, Currow DC, Frith P, Fazekas BS, McHugh A, Bui C (2003): Randomised, double blind, placebo controlled crossover trial of sustained release morphine for the management of refractory dyspnoea. BMJ $\underline{327(7414)}$, 523-528

Abernethy AP, McDonald CF, Frith PA, Clark K, Herndon JE 2nd, Marcello J, Young IH, Bull J, Wilcock A, Booth S, Wheeler JL, Tulsky JA, Crockett AJ, Currow DC (2010): Effect of palliative oxygen versus room air in relief of breathlessness in patients with refractory dyspnoea: a double-blind, randomised controlled trial. Lancet 376(9743), 784-93

Altman DG: Practical statistics for medical research, $1^{\text {st }}$ Edition, Chapman \& Hall, London 1991

American Thoracic Society (1999): Dyspnea. Mechanisms, assessment and management: a consensus statement. Am J Respir Crit Care Med 159(1), 321-340

Barbera L, Taylor C, Dudgeon D (2010): Why do patients with cancer visit the emergency department near the end of life? CMAJ 182(6), 563-568

Bausewein C, Farquhar M, Booth S, Gysels M, Higginson IJ (2007): Measurement of breathlessness in advanced disease: a systematic review. Respir Med 101(3), 399-410

Bausewein C, Booth S, Gysels M, Higginson I (2008 a): Non-pharmacological interventions for breathlessness in advanced stages of malignant and non-malignant diseases. Cochrane Database Syst Rev 2, CD005623

Bausewein C, Booth S, Higginson IJ (2008 b): Measurement of dyspnoea in the clinical rather than the research setting. Curr Opin Support Palliat Care 2(2), 95-99

Bausewein C, Booth S, Gysels M, Kühnbach R, Haberland B, Higginson IJ (2010 a): Individual breathlessness trajectories do not match summary trajectories in advanced cancer and chronic obstructive pulmonary disease: results from a longitudinal study. Palliat Med 24(8), 777-786

Bausewein C, Booth S, Gysels M, Kühnbach R, Haberland B, Higginson IJ (2010 b): Understanding breathlessness: cross-sectional comparison of symptom burden and palliative care needs in chronic obstructive pulmonary disease and cancer. J Palliat Med 13(9), 1109-1118

Booth S, Silvester S, Todd C (2003): Breathlessness in cancer and chronic obstructive pulmonary disease: using a qualitative approach to describe the experience of patients and carers. Palliat Support Care 1(4), 337-344

Booth S, Moosavi SH, Higginson IJ (2008): The etiology and management of intractable breathlessness in patients with advanced cancer: a systematic review of pharmacological therapy. Nat Clin Pract Oncol 5(2), 90-100

Borgsteede SD, Deliens L, Beentjes B, Schellevis F, Stalman WA, Van Eijk JT, Van der Wal G (2007): Symptoms in patients receiving palliative care: a study on patient-physician encounters in general practice. Palliat Med 21(5), 417-423

Bruera E, Schmitz B, Pither J, Neumann CM, Hanson J (2000): The frequency and correlates of dyspnea in patients with advanced cancer. J Pain Symptom Manage 19(5), 357-362 
Burt J, Shipman C, Richardson A, Ream E, Addington-Hall J (2010): The experiences of older adults in the community dying from cancer and non-cancer causes: a national survey of bereaved relatives. Age Ageing 39(1), 86-91

Campbell ML, Templin T, Walch J (2009): Patients who are near death are frequently unable to self-report dyspnea. J Palliat Med 12(10), 881-884

Chan KS, Sham MMK, Tse DMW, Thorsen AB: Palliative medicine in malignant respiratory diseases; in: Oxford Textbook of Palliative Medicine, $3^{\text {rd }}$ Edition, hrsg. v. Doyle D, Hanks G u. a. Oxford University Press, Oxford 2004, 593

Christakis NA, Lamont EB (2000): Extent and determinants of error in doctors' prognoses in terminally ill patients: prospective cohort study. West J Med 172(5), 310-313

Cohen J: Statistical Power Analysis for the Behavioral Sciences, 2. Auflage, Lawrence Erlbaum Associates, Hillsdale 1988

Conill C, Verger E, Henríquez I, Saiz N, Espier M, Lugo F, Garrigos A (1997): Symptom prevalence in the last week of life. J Pain Symptom Manage 14(6), 328-331

Cranston JM, Crockett A, Currow D (2008): Oxygen therapy for dyspnoea in adults. Cochrane Database Syst Rev $\underline{3}$, CD004769.

Cuervo Pinna MA, Mota Vargas R, Redondo Moralo MJ, Sánchez Correas MA, Pera Blanco G (2009): Dyspnea--a bad prognosis symptom at the end of life. Am J Hosp Palliat Care 26(2), 89-97

Currow DC, Agar M, Smith J, Abernethy AP (2009 a): Does palliative home oxygen improve dyspnoea? A consecutive cohort study. Palliat Med 23(4), 309-316

Currow DC, Plummer JL, Crockett A, Abernethy AP (2009 b): A community population survey of prevalence and severity of dyspnea in adults. J Pain Symptom Manage 38(4), 533-545

Currow DC, Smith J, Davidson PM, Newton PJ, Agar MR, Abernethy AP (2010): Do the trajectories of dyspnea differ in prevalence and intensity by diagnosis at the end of life? A consecutive cohort study. J Pain Symptom Manage 39(4), 680-690

Delgado-Guay M, Parsons HA, Li Z, Palmer JL, Bruera E (2009): Symptom distress in advanced cancer patients with anxiety and depression in the palliative care setting. Support Care Cancer 17(5), $573-579$

Dorman S, Byrne A, Edwards A (2007): Which measurement scales should we use to measure breathlessness in palliative care? A systematic review. Palliat Med 21(3), 177-191

Dorman S, Jolley C, Abernethy A, Currow D, Johnson M, Farquhar M, Griffiths G, Peel T, Moosavi S, Byrne A, Wilcock A, Alloway L, Bausewein C, Higginson I, Booth S (2009): Researching breathlessness in palliative care: consensus statement of the National Cancer Research Institute Palliative Care Breathlessness Subgroup. Palliat Med 23(3) 213-27

Dudgeon DJ, Lertzman M (1998): Dyspnea in the advanced cancer patient. J Pain Symptom Manage $\underline{16(4)}, 212-219$

Dudgeon DJ, Kristjanson L, Sloan JA, Lertzman M, Clement K (2001): Dyspnea in cancer patients: prevalence and associated factors. J Pain Symptom Manage 21(2), 95-102 
Epstein AM, Hall JA, Tognetti J, Son LH, Conant L (1989): Using proxies to evaluate quality of life. Can they provide valid information about patients' health status and satisfaction with medical care? Med Care 27(3), 91-98

Ewing G, Rogers M, Barclay S, McCabe J, Martin A, Campbell M, Todd C (2006): Palliative care in primary care: a study to determine whether patients and professionals agree on symptoms. $\mathrm{Br} \mathbf{J}$ Gen Pract 56(522), 27-34

Galbraith S, Fagan P, Perkins P, Lynch A, Booth S (2010): Does the use of a handheld fan improve chronic dyspnea? A randomized, controlled, crossover trial. J Pain Symptom Manage 39(5), 831-8

Glare PA, Sinclair CT (2008): Palliative medicine review: prognostication. J Palliat Med 11(1), 84103

Graubner B: ICD-10-GM 2011, Systematisches Verzeichnis, Internationale statistische Klassifikation der Krankheiten und verwandter Gesundheitsprobleme, 10. Revision - German Modification, Deutscher Ärzte-Verlag 2010

Gripp S, Moeller S, Bölke E, Schmitt G, Matuschek C, Asgari S, Asgharzadeh F, Roth S, Budach W, Franz M, Willers R (2007): Survival prediction in terminally ill cancer patients by clinical estimates, laboratory tests and self-rated anxiety and depression. J Clin Oncol 25(22), 33133320 .

Gysels M, Bausewein C, Higginson IJ (2007): Experiences of breathlessness: a systematic review of the qualitative literature. Palliat Support Care 5(3), 281-302

Hayes AW, Philip J, Spruyt OW (2006): Patient reporting and doctor recognition of dyspnoea in a comprehensive cancer centre. Intern Med J $\underline{36(6)}, 381-384$

Heffernan C, Jenkinson C, Holmes T, Macleod H, Kinnear W, Oliver D, Leigh N, Ampong MA (2006): Management of respiration in MND/ALS patients: an evidence based review. Amyotroph Lateral Scler 7(1), 5-15

Henoch I, Bergman B, Gustafsson M, Gaston-Johansson F, Danielson E (2007): The impact of symptoms, coping capacity and social support on quality of life experience over time in patients with lung cancer. J Pain Symptom Manage 34(4), 370-379

Henoch I, Bergman B, Gustafsson M, Gaston-Johansson F, Danielson E (2008): Dyspnea experience in patients with lung cancer in palliative care. Eur J Oncol Nurs $\underline{12(2)}$, 86-96

Herold G: Innere Medizin, Selbstverlag, Köln 2009

Heyse-Moore LH, Ross V, Mullee MA (1991): How much of a problem is dyspnea in advanced cancer? Palliat Med 5, 20-26

Heyse-Moore L, Beynon T, Ross V (2000): Does spirometry predict dyspnoea in advanced cancer? Palliat Med 14(3), 189-195

Higginson I (1993): Palliative care: a review of past changes and future trends. J Public Health Med $\underline{15(1)}, 3-8$

Ho SF, O'Mahony MS, Steward JA, Breay P, Buchalter M, Burr ML (2001): Dyspnoea and quality of life in older people at home. Age Ageing $\underline{30(2)}$, 155-159

Hospiz- und Palliativerhebung: http://www.hope-clara.de/download/GesamtberichtHOPE2008.pdf, Abfragedatum: 20.09.2011 
Jennings AL, Davies AN, Higgins JP, Broadley K (2001): Opioids for the palliation of breathlessness in terminal illness. Cochrane Database Syst Rev 4, CD002066

Klaschik E: Palliativmedizin; in: Palliativmedizin - Grundlagen und Praxis, 5. Auflage; hrsg. v. Husebø S und Klaschik E; Springer Medizin Verlag, Heidelberg 2009; 1-45

Klaschik E, Nauck F (1998): Historische Entwicklung der Palliativmedizin. Zentralbl Chir $\underline{123}$, 620623

Kolodziej MA, Jensen L, Rowe B, Sin D (2007): Systematic review of non-invasive positive pressure ventilation in severe stable COPD. Eur Respir J 30(2), 293-306

Krumm N, Stiel S, Ostgathe C, Lindena G, Nauck F, Elsner F, Radbruch L und die Koordinierungsgruppe HOPE (2008): Subjektives Befinden bei Palliativpatienten - Ergebnisse der Hospiz- und Palliativerhebung (HOPE). Z Palliativmed 9, 132-138

Lanken PN, Terry PB, Delisser HM, Fahy BF, Hansen-Flaschen J, Heffner JE, Levy M, Mularski RA, Osborne ML, Prendergast TJ, Rocker G, Sibbald WJ, Wilfond B, Yankaskas JR; ATS End-of-Life Care Task Force (2008): An official American Thoracic Society clinical policy statement: palliative care for patients with respiratory diseases and critical illnesses. Am J Respir Crit Care Med. 177(8), 912-927

Leupoldt A, Sommer T, Kegat S, Baumann HJ, Klose H, Dahme B, Büchel C (2009): Dyspnea and pain share emotion-related brain network. Neuroimage 48(1), 200-206

Linsenmeier C: Prevalence of breathlessness in patients with advanced cancer - depending on primary site and measurement tools? A systematic review. MSc Thesis, King's College London, UK 2008

Maltoni M, Pirovano M, Scarpi E, Marinari M, Indelli M, Arnoldi E, Gallucci M, Frontini L, Piva L, Amadori D (1995): Prediction of survival of patients terminally ill with cancer. Results of an Italian prospective multicentric study. Cancer $\underline{75(10)}, 2613-2622$

Maltoni M, Caraceni A, Brunelli C, Broeckaert B, Christakis N, Eychmueller S, Glare P, Nabal M, Vigano A, Larkin P, De Conno F, Hanks G, Kaasa S; Steering Committee of the European Association for Palliative Care (2005): Prognostic factors in advanced cancer patients: evidencebased clinical recommendations - a study by the Steering Committee of the European Association for Palliative Care. J Clin Oncol 23(25), 6240-6248

Manning HL, Schwartzstein RM (1995): Pathophysiology of dyspnea. N Engl J Med 333(23), 15471553

McMillan SC, Small BJ (2002): Symptom distress and quality of life in patients with cancer newly admitted to hospice home care. Oncol Nurs Forum 29(10), 1421-1428

Moody LE, McMillan S (2003): Dyspnea and quality of life indicators in hospice patients and their caregivers. Health Qual Life Outcomes 17, 1-9

Morita T, Tsunoda J, Inoue S, Chihara S (1999): The Palliative Prognostic Index: a scoring system for survival prediction of terminally ill cancer patients. Support Care Cancer 7(3), 128-133

Nauck F: Symptome und Symptomkontrolle bei Patienten in der Finalphase auf einer Palliativstation. Med. Diss. Bonn 2003 
Nauck F, Ostgathe C, Klaschik E, Bausewein C, Fuchs M, Lindena G, Neuwöhner K, Schulenberg D, Radbruch L, Working Group on the Core Documentation for Palliative Care Units in Germany (2004): Drugs in palliative care: results from a representative survey in Germany. Palliat Med 18(2), 100-107

Nekolaichuk CL, Bruera E, Spachynski K, MacEachern T, Hanson J, Maguire TO (1999 a): A comparison of patient and proxy symptom assessments in advanced cancer patients. Palliat Med 13(4), 311-323

Nekolaichuk CL, Maguire TO, Suarez-Almazor M, Rogers WT, Bruera E (1999 b): Assessing the reliability of patient, nurse and family caregiver symptom ratings in hospitalized advanced cancer patients. J Clin Oncol 17(11), 3621-3630

Neuman A, Gunnbjörnsdottir M, Tunsäter A, Nyström L, Franklin KA, Norrman E, Janson C (2006): Dyspnea in relation to symptoms of anxiety and depression: A prospective population study. Respir Med 100(10), 1843-1849

Nishimura K, Izumi T, Tsukino M, Oga T (2002): Dyspnea is a better predictor of 5-year survival than airway obstruction in patients with COPD. Chest 121(5), 1434-1440

Oi-Ling K, Man-Wah DT, Kam-Hung DN (2005): Symptom distress as rated by advanced cancer patients, caregivers and physicians in the last week of life. Palliat Med 19(3), 228-233

Oken MM, Creech RH, Tormey DC, Horton J, Davis TE, McFadden ET, Carbone PP (1982): Toxicity and response criteria of the Eastern Cooperative Oncology Group. Am J Clin Oncol 5(6), 649-655.

Ostgathe C, Alt-Epping B, Golla H, Gaertner J, Lindena G, Radbruch L, Voltz R, Hospice and Palliative Care Evaluation (HOPE) Working Group (2011): Non-cancer patients in specialized palliative care in Germany: what are the problems? Palliat Med 25(2), 148-152

Pakhomov SV, Jacobsen SJ, Chute CG, Roger VL (2008): Agreement between patient-reported symptoms and their documentation in the medical record. Am J Manag Care 14(8), 530-539

Pallant J: SPSS Survival manual., $3^{\text {rd }}$ Edition, Open University Press, Berkshire 2007

Pirovano M, Maltoni M, Nanni O, Marinari M, Indelli M, Zaninetta G, Petrella V, Barni S, Zecca E, Scarpi E, Labianca R, Amadori D, Luporini G (1999): A new palliative prognostic score: a first step for the staging of terminally ill cancer patients. Italian Multicenter and Study Group on Palliative Care. J Pain Symptom Manage 17(4), 231-239

Potter J, Hami F, Bryan T, Quigley C (2003): Symptoms in 400 patients referred to palliative care services: prevalence and patterns Palliat Med. 17(4), 310-314

Radbruch L, Nauck F, Aulbert E: Grundlagen der Palliativmedizin, Definition, Entwicklung und Ziele; in: Lehrbuch der Palliativmedizin, 2. Auflage, hrsg. v. Aulbert E, Nauck F, Radbruch L, Schattauer Verlag, Stuttgart 1997, 1-14

Radbruch L, Sabatowski R, Loick G, Jonen-Thielemann I, Elsner F, Hörmann E (2000 a): MIDOS - Validierung eines minimalen Dokumentationssystems für die Palliativmedizin. Schmerz $\underline{14(4)}, 231-239$

Radbruch L, Sabatowski R, Loick G, Jonen-Thielemann I, Kasper M, Gondek B, Lehmann KA, Thielemann I (2000 b): Cognitive impairment and its influence on pain and symptom assessment in a palliative care unit: development of a Minimal Documentation System. Palliat Med 14(4), 266-276 
Radbruch L, Nauck F, Ostgathe C, Elsner F, Bausewein C, Fuchs M, Lindena G, Neuwöhner K, Schulenberg D (2003): What are the problems in palliative care? Results from a representative survey. Support Care Cancer 11(7), 442-451

Radbruch L, Ostgathe C, Elsner F, Nauck F, Bausewein C, Fuchs M, Lindena G, Neuwöhner K, Schulenberg D (2004): Prozesse und Interventionen auf den deutschen Palliativstationen. Ergebnisse der Kerndokumentation 2001. Schmerz 18(3), 179-188

Radbruch L, Nauck F, Ostgathe C, Lindena G: HOPE: Handbuch zu Dokumentation und Qualitätsmanagement in der Hospiz- und Palliativversorgung. der hospiz verlag, Ludwigsburg 2009

Reuben DB, Mor V (1986): Dyspnea in terminally ill cancer patients. Chest 89(2), 234-236

Simon ST, Bausewein C (2009): Management of refractory breathlessness in patients with advanced cancer. Wien Med Wochenschr 159(23-24), 591-598

Simon ST, Higginson IJ, Booth S, Harding R, Bausewein C (2010): Benzodiazepines for the relief of breathlessness in advanced malignant and non-malignant diseases in adults. Cochrane Database Syst Rev $\underline{1}, \mathrm{CD} 007354$

Smith EL, Hann DM, Ahles TA, Furstenberg CT, Mitchell TA, Meyer L, Maurer LH, Rigas J, Hammond S (2001): Dyspnea, anxiety, body consciousness, and quality of life in patients with lung cancer. J Pain Symptom Manage 21(4), 323-329

Sneeuw KC, Aaronson NK, Sprangers MA, Detmar SB, Wever LD, Schornagel JH (1999): Evaluating the quality of life of cancer patients: assessments by patients, significant others, physicians and nurses. Br J Cancer $\underline{81(1)}$, 87-94

Solano JP, Gomes B, Higginson IJ (2006): A comparison of symptom prevalence in far advanced cancer, AIDS, heart disease, chronic obstructive pulmonary disease and renal disease. J Pain Symptom Manage $\underline{31(1)}, 58-69$

Stephens RJ, Hopwood P, Girling DJ, Machin D (1997): Randomized trials with quality of life endpoints: are doctors' ratings of patients' physical symptoms interchangeable with patients' selfratings? Qual Life Res $\underline{6(3)}$, 225-236

Stiel S, Bertram L, Neuhaus S, Nauck F, Ostgathe C, Elsner F, Radbruch L (2010 a): Evaluation and comparison of two prognostic scores and the physicians' estimate of survival in terminally ill patients. Support Care Cancer 18(1), 43-49

Stiel S, Matthes ME, Bertram L, Ostgathe C, Elsner F, Radbruch L (2010 b):Validation of the new version of the minimal documentation system (MIDOS) for patients in palliative care : the German version of the edmonton symptom assessment scale (ESAS). Schmerz 24(6), 596-604

Stiel S, Pulst K, Krumm N, Ostgathe C, Nauck N, Lindena G, Radbruch L $(2010$ c): Palliativmedizin im Spiegel der Zeit - Ein Verglich der Ergebnisse der Hospiz- und Palliativerhebungen von 2004 und 2009. Zeitschrift für Palliativmedizin 11, 78-84

Stroemgren AS, Groenvold M, Pedersen L, Olsen AK, Spile M, Sjogren P (2001 a): Does the medical record cover the symptoms experienced by cancer patients receiving palliative care? A comparison of the record and patient self-rating. J Pain Symptom Manage 21, 189-196

Stroemgren AS, Groenvold M, Sorensen A, Andersen L (2001 b): Symptom recognition in advanced cancer. A comparison of nursing records against patient self-rating. Acta Anaesthesiol Scand 45, 1080-1085 
Sullivan MJ, Green HJ, Cobb FR (1990): Skeletal muscle biochemistry and histology in ambulatory patients with long-term heart failure. Circulation 81(2), 518-527

Tataryn D, Chochinov HM (2002): Predicting the trajectory of will to live in terminally ill patients. Psychosomatics $\underline{43(5)}, 370-377$

Temel JS, Greer JA, Muzikansky A, Gallagher ER, Admane S, Jackson VA, Dahlin CM, Blinderman CD, Jacobsen J, Pirl WF, Billings JA, Lynch TJ (2010): Early palliative care for patients with metastatic non-small-cell lung cancer. N Engl J Med 363(8), 733-742

Teunissen SC, de Graeff A, de Haes HC, Voest EE (2006): Prognostic significance of symptoms of hospitalised advanced cancer patients. Eur J Cancer 42(15), 2510-2516

Tishelman C, Petersson LM, Degner LF, Sprangers MA (2007): Symptom prevalence, intensity, and distress in patients with inoperable lung cancer in relation to time of death. J Clin Oncol 25(34), $5381-5389$

Vigano A, Dorgan M, Buckingham J, Bruera E, Suarez-Almazor ME (2000): Survival prediction in terminal cancer patients: a systematic review of the medical literature. Palliat Med 14(5), 363-374

Vitetta L, Kenner D, Kissane D, Sali A (2001): Clinical outcomes in terminally ill patients admitted to hospice care: diagnostic and therapeutic interventions. J Palliat Care 17(2), 69-77

Walsh D, Rybicki L (2006): Symptom clustering in advanced cancer. Support Care Cancer 14(8), 831-836

Walsh D, Donnelly S, Rybicki L (2000): The symptoms of advanced cancer: relationship to age, gender, and performance status in 1,000 patients. Support Care Cancer 8(3), 175-179

Wegweiser Hospiz- und Palliativmedizin Deutschland: http://www.wegweiser-hospizpalliativmedizin.de/, Abfragedatum: 20.11.2011

World Health Organization. WHO Definition of Palliative Care.

http://www.who.int/cancer/palliative/definition/en/ Letzter Zugriff: 24.09.2011

Zimmermann C, Burman D, Follwell M, Wakimoto K, Seccareccia D, Bryson J, Le LW, Rodin G (2010): Predictors of symptom severity and response in patients with metastatic cancer. Am J Hosp Palliat Care 27(3), 175-181

Zloklikovits S, Andritsch E, Fröhlich B, Verebes J, Dietmaier G, Samonigg H (2005): Assessing symptoms of terminally-ill patients by different raters: a prospective study. Palliat Support Care 3(2), $87-98$ 


\section{Veröffentlichungen}

Teile der Ergebnisse der vorliegenden Dissertation wurden bereits veröffentlicht:

\section{1) Vorträge:}

Altfelder N, Nauck F, Alt-Epping B, Bausewein C, Simon ST (2010): Characteristics of patients with breathlessness - a German national survey on palliative care in-patient units. Zitiert nach Inhaltsangabe des Vortrags (gehalten 11.06.2010) in: $6^{\text {th }}$ Research Congress of the European Association for Palliative Care, Glasgow, United Kingdom, 10. bis 12. Juni 2010, Palliative Medicine $\underline{24(4)}, 37$

Der Vortrag wurde mit dem "Abstract Plenary Award” ausgezeichnet.

Simon ST, Altfelder N, Alt-Epping B, Bausewein C, Nauck F (2010): Is breathlessness what the patient says it is? A German national survey comparing patient and proxy assessments. Zitiert nach Inhaltsangabe des Vortrags (gehalten 10.06.2010) in: $6^{\text {th }}$ Research Congress of the European Association for Palliative Care, Glasgow, United Kingdom, 10. bis 12. Juni 2010, Palliative Medicine 24(4), 29

2) Poster:

Altfelder N, Nauck F, Alt-Epping B, Ostgathe C, Bausewein C, Simon ST (2010): Charakteristika von Palliativpatienten mit Atemnot - Ergebnisse der Hospiz- und Palliativerhebungen (HOPE) von 2006 bis 2008. Zitiert nach Inhaltsangabe des Posters in: 8. Kongress der Deutschen Gesellschaft für Palliativmedizin, Dresden, 9. bis 11. September 2010, Zeitschrift für Palliativmedizin 11, 243

Simon ST, Altfelder N, Alt-Epping B, Ostgathe C, Bausewein C, Nauck F (2010): Sieht man einem Patienten seine Atemnot an? Analyse der HOPE-Daten 2006-2008 mit einem Vergleich zwischen Selbst- und Fremdeinschätzung. Zitiert nach Inhaltsangabe des Posters in: 8. Kongress der Deutschen Gesellschaft für Palliativmedizin, Dresden, 9. bis 11. September 2010, Zeitschrift für Palliativmedizin 11,243

Weitere Veröffentlichungen der Promovendin zum Thema der Doktorarbeit:

Alt-Epping B, Stäritz A, Simon ST, Altfelder N, Lindena G, Nauck F: What is special about patients with lung cancer and pulmonary metastases in palliative care? Results from a nationwide survey. Journal of Palliative Medicine, eingereicht am 13.07.2011

Simon ST, Voltz R, Altfelder N, Gaertner J (2011): Therapie der refraktären Atemnot bei Patienten mit fortgeschrittenen Erkrankungen - evidence-based update für den klinischen Alltag. Therapeutische Umschau, im Druck

Stäritz A, Alt-Epping B, Altfelder N, Simon ST, Lindena G, Nauck F (2010): Was ist das Besondere bei der Palliativversorgung von Patienten mit malignen pulmonalen Erkrankungen? Symptommuster im Vergleich (HOPE 2006-2008). Zitiert nach Inhaltsangabe des Posters in: 8. Kongress der Deutschen Gesellschaft für Palliativmedizin, Dresden, 9. bis 11. September 2010, Zeitschrift für Palliativmedizin 11, 243

Stäritz A, Alt-Epping B, Altfelder N, Simon ST, Lindena G, Nauck F (2010): Disease specific symptom prevalences in patients with lung cancer and pulmonary metastases - staff assessment versus self assessment. Results from a multicenter survey. Zitiert nach Inhaltsangabe des Vortrags (gehalten 11.06.2010) in: $6^{\text {th }}$ Research Congress of the European Association for Palliative Care, Glasgow, United Kingdom, 10. bis 12. Juni 2010, Palliative Medicine 24(4), 13 


\section{Danksagung}

Herrn Prof. Dr. med. F. Nauck danke ich sehr herzlich für die Überlassung des interessanten Dissertationsthemas. Darüber hinaus waren mir seine Anregungen, Ratschläge und konstruktive Kritik eine große Hilfe bei der Fertigstellung dieser Arbeit. Insbesondere aber seine unkomplizierte Art der Kommunikation, seine respektvollen und anerkennenden Worte sowie seine Verlässlichkeit waren für mich von unschätzbarem Wert und sind in der Tat nicht selbstverständlich.

Mein besonderer Dank gilt Dr. med. S. Simon, der die Arbeit initiiert, strukturiert und mit besonderem Engagement betreut hat. Seine Freude an der Wissenschaft, sein umfassendes Fachwissen und sein unermüdliches Streben, laufende Projekte voranzutreiben und neue zu initiieren, waren und sind für mich beispielhaft. Seiner unendlichen Geduld und seiner bemerkenswerten Fähigkeit zur Motivation schulde ich viel. Er hat ganz wesentlich zum Gelingen dieser Arbeit beigetragen.

Frau Dr. med. C. Bausewein möchte ich danken für ihre allzeit prompte und ausgezeichnete Beratung in allen wissenschaftlichen und formalen Angelegenheiten, v. a. aber auch für Ihre freundlichen Worte. Herrn Dr. med. B. Alt-Epping danke ich für seine allzeit selbstlose Hilfe und die vielen nützlichen Tipps, die mir meine Arbeit stets erleichtert haben.

Danken möchte ich auch Frau Dr. G. Lindena-Gläss für die Überlassung der Daten von HOPE sowie für ihre Unterstützung bei der Zusammenführung der unterschiedlichen Datensätze. Frau Dr. Gao Wei gilt mein Dank für ihre zuverlässige und höchst kompetente Hilfe in allen statistischen Belangen. Ich danke Herrn J. Roß für die sofortige Hilfeleistung bei der Ermittlung der aktuellen Zahlen für die spezialisierten stationären Palliativeinrichtungen in Deutschland.

Frau A. Stäritz danke ich für die gute Zusammenarbeit und Frau Dr. med. S. Rühling für ihre freundschaftliche Unterstützung nicht nur bei der äußeren Form. Meinem Ehemann gilt mein Dank für seine ständige Diskussionsbereitschaft, das Korrekturlesen und sein Verständnis. Besonders danke ich auch meinen Eltern, die mich zu jeder Zeit ermutigt und unterstützt haben. Insbesondere wäre ohne ihren Einsatz in der Kinderbetreuung, meine Teilnahme an diversen Pflichtterminen und Arbeitstreffen nur unter sehr komplizierten Umständen möglich gewesen.

Zuletzt möchte ich auch den Organisatoren von HOPE sowie allen an HOPE teilnehmenden Einrichtungen danken, ohne deren Engagement meine Arbeit nicht hätte entstehen können. 


\section{Lebenslauf}

Mein Name ist Nadine Altfelder, geb. Huhnold. Ich wurde am 17. September 1976 in Göttingen geboren. Nach dem Abitur 1996 am Felix-Klein-Gymnasium in Göttingen begann ich im gleichen Jahr das Studium der Humanmedizin an der Universität Göttingen. Dieses konnte ich 2003 erfolgreich mit dem 3. Staatsexamen abschließen.

Im August 2003 begann ich meine Weiterbildung zunächst als Ärztin im Praktikum in der Inneren Abteilung des Evangelischen Krankenhauses in Oldenburg. Im Oktober 2004 wurde mir die Vollapprobation erteilt. Im März 2005 wechselte ich aus familiären Gründen in die Innere Abteilung des Krankenhauses in Weinheim.

Im April 2008 ist unser Sohn Moritz geboren. Nach der Elternzeit arbeitete ich von März 2009 bis September 2010 Teilzeit in der Kardiologischen Gemeinschaftspraxis Prof. Dr. Bär und Dr. Dr. Kuhn in Heidelberg. Im Oktober 2010 kehrte ich in Vollzeit ans Krankenhaus in Weinheim zurück.

Im Januar 2011 wurde unsere Tochter Lotte geboren. Derzeit befinde ich mich erneut in Elternzeit. Geplant ist die Wiederaufnahme meiner Tätigkeit am Krankenhaus in Weinheim im Frühjahr 2012, um im gleichen Jahr den Facharzt für Innere Medizin zu erlangen.

Seit 2008 bin ich Doktorandin in der Abteilung Palliativmedizin im Zentrum Anaesthesiologie, Rettungs- und Intensivmedizin der Medizinischen Fakultät der Universität Göttingen. 